\title{
Hochauflösende Untersuchung der lokalen Wasserstoffverteilung in metallischen Multischichten mit Hilfe der tomographischen Atomsonde und der Sekundärionenmassenspektrometrie
}

\author{
Dissertation \\ zur Erlangung des Doktorgrades \\ der Mathematisch-Naturwissenschaftlichen Fakultäten \\ der Georg-August-Universität zu Göttingen
}

vorgelegt von

Philipp Kesten

aus Berlin

Göttingen 2000 
D7

Referent: Prof. Dr. R. Kirchheim

Korreferent: Prof. Dr. W. Felsch

Tag der mündlichen Prüfung: 22.06.2000 


\section{Inhaltsverzeichnis}

1 Einleitung 1

2 Grundlagen der Wasserstofflöslichkeit 5

2.1 Wasserstofflöslichkeit in Metallen . . . . . . . . . . . . . 5

2.2 Wasserstoff in Multischichten . . . . . . . . . . . . . 8

2.3 Verwendete Metall-H-Systeme . . . . . . . . . . . . . . . . 10

2.3.1 Untersuchte Metallgrenzflächen . . . . . . . . . . . . . 11

2.3.2 Phasendiagramme der Metall-H-Systeme . . . . . . . . . . 12

2.3.3 Binäre Phasendiagramme der Metallsysteme . . . . . . . . 14

3 Chemische Analysetechniken $\quad \mathbf{1 5}$

3.1 Feldionenmikroskop (FIM) und Tomographische Atomsonde (TAP) 15

3.1.1 Feldionenmikroskop . . . . . . . . . . . . . 15

3.1 .2 Tomographische Atomsonde . . . . . . . . . . . . . 16

3.2 Sekundärionenmassenspektrometer (SIMS) . . . . . . . . . . . 20

4 Metallische Schichtsysteme $\quad 25$

4.1 Herstellungsmethoden . . . . . . . . . . . . . . . . . . 25

4.1.1 Gepulste Laserdeposition (LD) . . . . . . . . . . . . 26

4.1.2 Ionenstrahlsputterdeposition (SD) . . . . . . . . . . . . 26

4.2 Präparation der Schichtsysteme . . . . . . . . . . . . . . . . . 27

4.2.1 Präparation für SIMS . . . . . . . . . . . . . . . . 27

4.2 .2 Präparation für FIM und TAP . . . . . . . . . . . . 28

4.3 Einfluß der Herstellungsmethode auf die

Grenzflächenschärfe der Multischichtsysteme . . . . . . . . . . . . 34

4.3 .1 Das System Pd/Nb . . . . . . . . . . . . . . . 34

4.3.2 Das System Fe/V . . . . . . . . . . . . . . . . 40

5 Wasserstoffverteilung in Multischichten 43

$5.1 \quad \mathrm{H}-$-D-Beladung . . . . . . . . . . . . . . . . . . . . . 43

5.1.1 D-Beladung aus der Gasphase . . . . . . . . . . . . . 43

5.1.2 Elektrochemische H-Beladung . . . . . . . . . . . . . . 44

5.2 Messung der H-Verteilung bei 300 K (SIMS-Analysen) . . . . . . 44 
5.3 Diskussion der Ergebnisse der Messungen bei 300 K . . . . . . . . 51

5.3 .1 Einfluß der H-Diffusion . . . . . . . . . . . . . . 52

5.4 Messung der D-Verteilung bei tiefen Temperaturen (TAP-Analysen) 60

$5.4 .1 \mathrm{Pd} / \mathrm{Nb}-$ Multischichten . . . . . . . . . . . . . 61

$5.4 .2 \mathrm{Nb} / \mathrm{W}$-Grenzfläche . . . . . . . . . . . . . . . 61

$5.4 .3 \mathrm{Fe} / \mathrm{V}-\mathrm{Schichtsysteme} \mathrm{\ldots ............} \mathrm{.} \mathrm{.} \mathrm{.} 66$

5.5 Diskussion der Ergebnisse der Messungen bei $60 \mathrm{~K}$. . . . . . . 71

5.5.1 Einfluß der D-Diffusion bei $60 \mathrm{~K}$. . . . . . . . . . . 71

5.6 Vergleich der H-/D-Segregation mit dem Modell von Züchner . . . 75

5.7 Interpretation der bei $20 \mathrm{~K}$ gemessenen D-Verteilung . . . . . . . 76

5.7.1 Dreidimensionale Rekonstruktion der TAP-Daten und Konzentrationsbestimmung . . . . . . . . . . 76

5.7.2 Diskussion der bei $20 \mathrm{~K}$ gemessenen D-Gleichgewichtsverteilung . . . . . . . . . . . . . . . . . 79

$\begin{array}{llr}6 & \text { Zusammenfassung } & 87\end{array}$

$\begin{array}{ll}\text { A H-Permeation durch Pd/Nb-Multischichten } & 90\end{array}$

A.1 Elektrochemische Doppelzelle . . . . . . . . . . . . . . . . . 91

A.2 H-Permeation durch Multischichten . . . . . . . . . . . . . . . 92

A.3 Diskussion der Ergebnisse . . . . . . . . . . . . . . . 95 


\section{Kapitel 1}

\section{Einleitung}

Durch den enormen Fortschritt in der Präparation und Analyse nanoskopischer Materialsysteme, wie z.B. dünner Schichten mit Schichtdicken bis hinunter zu einigen Atomlagen und Clustern aus weniger als 100 Atomen, ist auch das Verhalten des Wasserstoffes in solchen Systemen ein Feld intensiver Forschung geworden.

In Dünnschichtsystemen führt die Reduzierung der Schichtdicken in den Nanometerbereich im Vergleich mit den massiven Materialien zu sehr komplexen Mikrostrukturen, mit denen gerade die Wasserstoffatome als die kleinsten und beweglichsten unter den Elementen - positiv formuliert - besonders gerne wechselwirken. Die Komplexität der Mikrostruktur hat ihren Ursprung vor allem in der Tatsache, daß dünne Filme nur auf Substraten stabil sind. Zwischen Schicht und Substrat liegt somit stets eine Grenzfläche vor. Nötige Puffer- oder Deckschichten zur Stabilisierung der Schicht bzw. zum Schutz vor Oxidation oder Hydrierung sind aufgrund ähnlicher Dicke nicht mehr vernachlässigbar und führen zu weiteren Grenzflächen. Je dünner die Schichten, umso höher wird auch der Volumenanteil der Grenzflächen am Gesamtsystem.

Jede Grenzfläche ist für sich genommen schon ein mikrostrukturell schwer zu beschreibendes System. Durch die nötige Anpassung zentraler Eigenschaften der benachbarten Materialien wie Gitterstruktur und elektronischer Struktur über die Grenzfläche hinweg treten in der Regel hohe mechanische Spannungen und hohe Defektdichten auf. Der Grad der Komplexität steigt dabei mit der Anzahl der Schichten, der Anzahl unterschiedlicher Komponenten und abnehmender Schichtdicke im Multischichtsystem.

Andererseits zeigen gerade metallische Multischichtsysteme mit ihrer chemischen Modulation auf Nanometerskala interessante, von den massiven Materialien unbekannte Eigenschaften wie GMR [1], erhöhte Härte [2] und hohe Neutronenreflektivität [3].

Der Wasserstoff $(\mathrm{H})$ zeigt schon in dünnen Einfachschichten ein von massiven Metallen abweichendes Verhalten. Dies liegt vornehmlich an den besonderen Spannungszuständen, die je nach Herstellungsprozeß durch die Wechselwirkung mit dem Substrat entstehen. So zeigen Messungen von Zabel et al. [4, 5, 6] ei- 
ne Abnahme des Existenzbereiches des $\alpha$ - $\beta$-Zweiphasengebietes im Nb-H-System mit abnehmender Schichtdicke. Laudahn et al.[7] beobachten eine Veränderung des Existenzbereiches in Abhängigkeit des Herstellungsprozesses.

In einem Multischichtpaket sollten die H-Eigenschaften nun nicht nur von den Spannungszuständen in den beteiligten Einzelschichten, sondern auch von den unterschiedlichen H-Löslichkeiten der beteiligten Metalle abhängen. Hinzu kommen die Wechselwirkungen der Einzelschichten miteinander. Diese Grenzflächeneffekte sind vorallem:

- Anpassung der Gitter der Einzelschichten an den Grenzflächen bei kohärentem Aufwachsen, bzw. Einbau von Versetzungen in der Grenzfläche bei inkohärentem Wachstum,

- Elektronische Übergangsstrukturen an der Grenzfläche zwischen benachbarten Metallen und Elekronenübertritt ins elektronegativere Metall.

Beide Punkte sind für die H-Löslichkeit von entscheidenem Einfluß und bestimmen somit ebenfalls den Transport von Wasserstoff in Multischichtsystem.

Die Multischichtsysteme lassen sich bezüglich des H-Lösungsverhalten in zwei Kategorien unterteilen. Zum einen in Schichtsysteme, die aus Metallen mit wenig unterschiedlichem H-Lösungsverhalten bestehen. Messungen von Miceli, Zabel et al. an Nb/Ta-Multischichten fallen in diese Kategorie. Sie demonstrieren bei der Aufnahme von Wasserstoff strukturelle und thermodynamische Abweichungen der beteiligten Metallgitter im Vergleich zu den massiven Metallen [8, 9, 10, 11].

In die zweite Kategorie fallen Multischichtsysteme aus Mo/V [12], Fe/V [13], $\mathrm{Fe} / \mathrm{Nb}[14], \mathrm{Pd} / \mathrm{Nb}[15]$ und W/Nb. Sie zeichnen sich durch einen hohen HLöslichkeitsunterschied aus, der erwarten läßt, daß Wasserstoff fast ausschließlich im V bzw. Nb gelöst wird. Die zitierten Arbeiten enthalten jeweils Untersuchungen des Einflusses der H-Beladung auf Struktur, Spannungszustände und Phasenbildung in den Multischichtsystemen.

Bei diesen Systemen ist, zusätzlich zu den Spannungseffekten auch ein deutlicher Einfluß des elektronischen Effektes auf das H-Lösungsverhalten zu erwarten. So schließen Hjörvarsson et al. aus Messungen von H-beladenen Multischichten des V mit geringlösenden Zwischenschichten aus Mo [16] bzw. Fe [17] auf die Existenz sogenannter Dead-Layers, einen an Wasserstoff verarmten Bereich, der sich sowohl bei Mo- als auch bei Fe-Zwischenschichten ausgehend von den Grenzflächen 2-3 Atomlagen ins V erstreckt. Dies führen sie auf einen Elektronenübertrag vom Mo bzw. Fe ins V in der Nähe der Grenzfläche zurück, der das V dort chemisch dem Mo bzw. Fe ähnlicher werden läßt und somit auch die H-Löslichkeit reduziert [18, 19, 20, 21, 22].

Hjörversson et al. führen das Modell der Dead-Layers als Interpretation ihrer Ergebnisse von H-Konzentrationsmessungen an Mo/V- und Fe/V-Schichtsystemen ein. Diese Untersuchungen wurden mit der $\mathrm{N}^{15}$-Methode durchgeführt [23, 
24], bei der die H-Konzentration mit einem kernphysikalischen Zerfallsprozeß tiefensensitiv detektiert werden kann. Die H-Tiefenprofilierung wurde an Proben durchgeführt, deren Multischichten aus mehreren Teil-Schichtsystemen mit unterschiedlicher V-Schichtdicke bestand. Sie finden eine Abnahme der H-Konzentration mit abnehmender V-Schichtdicke. Durch die Einführung einer Verarmungszone an den Grenzflächen, deren Volumenanteil mit sinkender Schichtdicke zunimmt, lassen sich die gemessenen H-Konzentrationen erklären. Allerdings besitzt die $\mathrm{N}^{15}$-Methode nur eine Tiefenauflösung größer als $4 \mathrm{~nm}$, so daß in den untersuchten Schichtsystemen - mit Doppelschichtdicken kleiner als $4 \mathrm{~nm}$ - die gemessenen Profile mittlere Werte für die H-Konzentration im gesamten Schichtpaket einschließlich der Zwischenschichten darstellen. Die mittleren Werte wurden anschliessend in H-Konzentrationsprofile in den V-Schichten umgerechnet, wobei die Schichtdicken mit Röntgenmessungen bestimmt wurden und eine atomar scharfe Grenzfläche angenommen wurde.

In dieser Arbeit soll nun unter Verwendung alternativer Meßmethoden die lokale H-Verteilung in dünnen Multischichtsystemen näher untersucht werden. Dazu wurden die hochaufösenden, massenspektrometrischen Verfahren der analytischen Feldionenmikroskopie mit tomographischer Atomsonde (TAP) und der Sekundärionenmassenspektrometrie (SIMS) eingesetzt. Dabei sollte die mit weniger als $1 \mathrm{~nm}$ sehr gute Tiefenauflösung des TAP genutzt werden, um zum ersten Mal direkte H-Konzentrationsmessungen innerhalb einzelner dünner metallischer Schichten durchzuführen. Insbesondere lag das Interesse darin, die von Hjörvarsson et al. postulierten H-Verarmungszonen an Grenzflächen mit stark unterschiedlicher H-Löslichkeit nachzuweisen. SIMS und TAP bieten im Gegensatz zur $\mathrm{N}^{15}$-Methode die Möglichkeit, die H- und Metallkonzentrationen gleichzeitig zu messen. Dadurch kann die H-Konzentration simultan mit der Grenzflächenrauhigkeit gemessen werden, so daß es möglich sein sollte zu unterscheiden, ob eine $\mathrm{H}$-Verarmung auf elektronische Effekte oder auf eine Durchmischung an der Grenzfläche zurückzuführen ist.

Die beschriebenen Methoden werden zwar zur Untersuchung von metallischen Systemen erfolgreich eingesetzt, doch liegen bis dato relativ wenige Arbeiten an Metall-H-Systemen vor. Während Messungen mit SIMS an massiven Systemen wie Nb-D, V-D, $\mathrm{LaNi}_{5}$-D von Züchner et al. durchgeführt wurden [25, 26, 27], sind SIMS-Messungen an H-beladenen Dünn- und Multischichtsystemen bislang nicht durchgeführt worden. Auch mit dem TAP sind nach unserer Kenntnis keine Untersuchungen von Metall-H(D)-Systemen gemacht worden, weder an massiven Metallen noch an Multischichtsystemen. Diese Arbeit dient deswegen auch zur Bestimmung der Rahmenparameter, bei denen eine nanoskalige Analyse der Hbzw. D-Verteilung in Multischichtsystemen mit SIMS und TAP möglich wird.

Die folgende Arbeit gliedert sich wie folgt: Zunächst werden in Kapitel 2 die Grundlagen der H-Löslichkeit und -Diffusion in Metallen und die speziellen Eigenschaften des Wasserstoffs in den untersuchten Metallsystemen dargestellt. In Kapitel 3 folgt die Beschreibung der Analysetechniken. Kapitel 4 behandelt 
die Präparation und Charakterisierung der verwendeten Multischichtsysteme. Die Ergebnisse werden in Kapitel 5 vorgestellt und diskutiert. Schwerpunkt ist dabei zunächst die Bestimmung der Parameter, bei denen eine Analyse der H-Verteilung in den Multischichtsystemen möglich wird. Abschliessend werden dann die bei den geeigneten Parametern durchgeführten Untersuchungen dargestellt und die Ergebnisse insbesondere auf die lokale Wasserstoffverteilung an den Grenzflächen hin analysiert. 


\title{
Kapitel 2
}

\section{Grundlagen der Wasserstofflöslichkeit in Metallen und metallischen Multischichten}

\begin{abstract}
Als Ausgangspunkt für die Untersuchungen an dünnen metallischen Multischichtsystemen sollen in diesem Kapitel zunächst die Grundlagen der H-Löslichkeit in massiven Metallen beschrieben werden. Anschließend werden daraus ableitbare Eigenschaften des Wasserstoffs in Multischichsystemen erörtert. Außerdem werden die verwendeten Metalle und ihre Metall-H-Systeme vorgestellt.
\end{abstract}

\subsection{Wasserstofflöslichkeit in Metallen}

Mit dem Begriff der Lösung wird allgemein der Teilchenaustausch innerhalb eines Materialsystems beschrieben. Setzt man z.B. ein Metall einer Gasatmosphäre aus, so wird sich je nach Metall, umgebendem Gas und Parametern wie p, T etc. eine charakteristische Menge an Gas im Metall lösen. Betrachtet man nun Wasserstoff und ein ideales Metallgitter, so stellt sich im thermodynamischen Gleichgewicht ein konstanter Teilchenaustausch zwischen Wasserstoffgas und dem atomar im Metallzwischengitter gelösten Wasserstoff ein.

$$
H_{2} \rightleftharpoons 2 H_{\text {imMetall }}
$$

Eine gegebene Menge Wasserstoff im Gesamtsystem wird sich so verteilen, daß sich Gleichgewichtswerte des äußeren Wasserstoffdruckes und der Konzentration von Wasserstoff im Metall einstellen werden. Den quantitativen Zusammenhang zwischen Gleichgewichtsdruck $p_{H_{2}}$ und der Gleichgewichtskonzentration $c$ im Metall erhält man aus der Äquivalenz der chemischen Potentiale beider Phasen im Gleichgewicht. Das chemische Potential eines Wasserstoffatoms im gasförmigen 
Zustand für kleine Drücke ist [28]

$$
\frac{1}{2} \cdot \mu_{H_{2}}=\frac{1}{2} \cdot \mu_{H_{2}}^{0}+\frac{R T}{2} \cdot \ln \frac{p_{H_{2}}}{p_{H_{2}}^{0}}
$$

wobei $\mu_{H_{2}}^{0}$ und $p_{H_{2}}^{0}$ die Werte bei $25^{\circ} \mathrm{C}$ und einem Druck von 1013 mbar beschreiben. Das chemische Potential eines im Zwischengitter gelösten Wasserstoffatoms erhält man aus der statistischen Betrachtung der Besetzung eines idealen Zwischengitters unter Vernachlässigung von Wasserstoff-Wasserstoff-Wechselwirkungen (ideale Lösung) [29]

$$
\mu_{H_{M e}}=\mu_{H_{M e}}^{0}+R T \cdot \ln \frac{c}{1-c} .
$$

Der Term $(1-c)$ im Argument des Logarithmus beschreibt die Blockierung schon besetzter Plätze. Er kann für kleine Konzentrationen vernachlässigt werden, d.h.

$$
\mu_{H_{M e}}=\mu_{H_{M e}}^{0}+R T \cdot \ln c
$$

Im Gleichgewicht sind diese chemischen Potentiale gleich (Gl.2.2 = Gl.2.4)

$$
\ln c=\frac{1}{R T} \cdot\left(\frac{1}{2} \cdot \mu_{H_{2}}^{0}-\mu_{H_{M e}}^{0}\right)+\frac{1}{2} \cdot \ln \frac{p_{H_{2}}}{p_{H_{2}}^{0}} .
$$

Man erhält somit das Sievertssche Gesetz [30]

$$
c=S \cdot \sqrt{\frac{p_{H_{2}}}{p_{H_{2}}^{0}}} .
$$

Dabei hängt die Löslchkeit $S$ vom verwendeten Metall ab. Bei gegebenem äußerem H-Druck kann die gelöste H-Konzentration je nach Metall um viele Größenordnungen variieren, so löst z.B. Nb ca. $10^{4}$ mal mehr Wasserstoff als Pd. Die unterschiedliche Wasserstofflöslichkeit in den verschiedenen Metallen läßt sich thermodynamisch aus den Freien Lösungsenthalpien der Metalle für Wasserstoff berechnen. Die Freie Lösungsenthalpie $\Delta G^{0}=\Delta H^{0}-T \cdot \Delta S^{0}$ ist für zahlreiche Metalle berechnet bzw. experimentell bestimmt worden. Zusammenstellungen der Werte finden sich in [31],[32],[33]. Der Löslichkeitsunterschied $k$ zwischen zwei Metallen im Gleichgewicht berechnet sich dann mit Gl.2.4 und

$$
\begin{aligned}
\Delta G^{0} & =\mu_{H_{M e}}^{0}-\frac{1}{2} \mu_{H_{2}}^{0} \\
\text { zu } \quad k & :=\frac{c_{2}}{c_{1}}=\exp \left(\frac{\Delta G_{1}^{0}-\Delta G_{2}^{0}}{R T}\right) .
\end{aligned}
$$

In Tabelle 2.1 sind die in der Literatur angegebenen H-Löslichkeiten und der mit Gl. 2.8 berechnete auf das Pd bezogene Löslichkeitsunterschied aufgeführt. 


\begin{tabular}{|c|c|c|c|c|c|}
\hline & $\mathrm{H}(\mathrm{kJ} / \mathrm{mol})$ & $\mathrm{S}(\mathrm{J} / \mathrm{mol} / \mathrm{K})$ & $\begin{array}{r}\mathrm{G}(\mathrm{kJ} / \mathrm{mo}) \\
\text { at } 300 \mathrm{~K} \\
\end{array}$ & & $\begin{array}{c}\mathrm{k} \\
\left(=\mathrm{c}_{P d} / \mathrm{c}_{M e}\right) \\
\end{array}$ \\
\hline $\mathrm{Pd}$ & -10 & -51 & 5.3 & {$[31]$} & 1 \\
\hline $\mathrm{Nb}$ & $\begin{array}{c}-40 \\
-33 \ldots-38\end{array}$ & -65 & $-20,5$ & $\begin{array}{l}{[31]} \\
{[32]}\end{array}$ & $3 \cdot 10^{-5}$ \\
\hline $\mathbf{V}$ & $\begin{array}{c}-32 \\
-26 \ldots-33\end{array}$ & -61 & $-13,7$ & $\begin{array}{l}{[31]} \\
{[32]}\end{array}$ & $5 \cdot 10^{-4}$ \\
\hline Mo & $\begin{array}{c}+52 \\
+15 \ldots+46\end{array}$ & -45 & +66 & $\begin{array}{l}{[31]} \\
{[32]}\end{array}$ & $5 \cdot 10^{10}$ \\
\hline $\mathrm{Fe}$ & $\begin{array}{c}+24 \\
+21 \ldots+33\end{array}$ & -54 & +40 & $\begin{array}{l}{[31]} \\
{[32]}\end{array}$ & $1 \cdot 10^{6}$ \\
\hline W & $\begin{array}{l}+100 \\
+96\end{array}$ & -39 & +112 & $\begin{array}{l}{[31]} \\
{[32]}\end{array}$ & $4 \cdot 10^{18}$ \\
\hline
\end{tabular}

Tabelle 2.1: Freie Lösungsenergie und-entropie von Wasserstoff in ausgewählten Metallen. Der Löslichkeitsunterschied $k$ wurde mit Gl.2.8 berechnet und ist exemplarisch auf Pd bezogen.

Diese Betrachtungen galten alle für geringe H-Konzentrationen im Grenzfall der idealen Lösung. Für höhere H-Konzentrationen kommt es nicht nur aufgrund der Platzblockierungen zu Abweichungen vom Sievertsschen Verhalten, sondern auch durch den zunehmenden Einfluß von Wasserstoff-Wasserstoff-Wechselwirkungen. Geht man in einem einfachen Modell von einer linearen Konzentrationsabhängigkeit für diesen Wechselwirkungseinfluß aus [33], den man zum chemischen Potential der idealen Lösung addieren kann, so ergibt sich

$$
\mu_{H_{M e}}=\mu_{H_{M e}}^{0}+R T \cdot \ln \frac{c}{1-c}+\text { konst. } \cdot c \text {. }
$$

Hieraus erhält man analog zum Modell der regulären Lösung [34] für eine attraktive H-H-Wechselwirkung die Bildung einer zweiten, wasserstoffreichen Phase, einer so genannten Hydridphase.

In vielen Metall-Wasserstoff-Systemen, wie z.B. im Pd-H-System, unterscheidet sich das entstehende Metallhydrid nur durch eine höhere Wasserstoffkonzentration und einen entsprechend vergrößerten Gitterparameter von der $\alpha$-Phase. Demzufolge spricht man in einem solchen Fall auch von der $\alpha^{\prime}$-Phase. In einigen 
Systemen kommt es aber auch zu geordneten bzw. stöchiometrischen Phasen, wie z.B. im Falle des Vanadiumhydrids $\mathrm{V}_{2} \mathrm{H}$ im V-H-System, bei dem sich die Besetzung vom Tetraederplatz in der $\alpha$-Phase zum Oktaederplatz in der $\beta$-Phase des kubisch-raumzentrierten Wirtsgitters ändert [35]. In einigen Fällen führt die Hydridbildung sogar zu einer Strukturänderung des Wirtsgitters, so z.B. im Zr-HSystem, bei dem das Wirtsgitter in der $\alpha$-Phase eine hexagonal-dichtest gepackte und in der $\beta$-Phase eine kubisch-raumzentrierte Struktur aufweist [33].

Das Lösungsverhalten ändert sich wesentlich, wenn Abweichungen vom idealen Zwischengitter vorliegen. Insbesondere Gitterfehler bieten dem Wasserstoff tiefere Platzenergien als die Plätze im ungestörten Zwischengitter. Das führt bei der Lösung von Wasserstoff zur preferentiellen Besetzung dieser tiefenergetischen Plätze und somit zu einer Absenkung des chemischen Potentials bei der Beladung mit Wasserstoff im Vergleich zu defektfreien Gittern. So detektiert Maxelon [36] mit Hilfe der Neutronenstreuung in versetzungsreichem Pd eine erhöhte Löslichkeit von Wasserstoff und Deuterium in den Dilatationsfeldern der Versetzungslinien. Die Absenkung des Potentials kann zur Sondierung der Platzenergieverteilung benutzt werden. Das Lösungsverhalten von Wasserstoff in defektreichen und amorphen Materialien konnte von Kirchheim [29] durch Berücksichtigung geeigneter Platzenergieverteilungen und deren Besetzung nach der Fermi-Dirac-Statistik modelliert werden. Der Einfluß von defektreichen Gittern auf das H-Diffusionsverhalten wurde ebenfalls von Kirchheim [29] beschrieben. Dabei bieten Gitterdefekte zum einen tiefenergetische Plätze, an denen ein Teil des Wasserstoffs gebunden wird, der somit nicht mehr zur weitreichenden Diffusion beitragen kann, was den Diffusionskoeffizienten absenkt. Zum anderen bieten Defekte wie Korngrenzen, die im Gitter ein Netzwerk bilden und deren Struktur offener als die des Gitters ist, auch schnellere Pfade für die H-Diffusion. Die beschleunigte Diffusion in Korngrenzen konnte u.a. von Mütschele an nanokristallinem Pd gezeigt werden [37]. Zur späteren Diskussion der H-Transportvorgänge in metallischen Multischichten sind in Tabelle $2.2 \mathrm{D}_{0}$ und die Aktivierungsenergie Q zur Berechnung des Diffusionskoeffizienten von Wasserstoff bei $300 \mathrm{~K}$ von ausgewählten massiven Metallen aufgeführt.

\subsection{Wasserstoff in Multischichten}

In einem metallischen Multischichtsystem mit seinen unterschiedlichen Komponenten und hohem Grenzflächenanteil erwartet man eine sehr komplexe Platzenergieverteilung und demzufolge ein entsprechend kompliziertes Lösungsverhalten des H. Geht man in einem idealen Modell von perfekten Gittern der beteiligten Metalle und defektfreien Grenzflächen aus, so bildet schon die abwechselnde Anordnung verschiedener Metalle eine periodische Potentiallandschaft, die in Abb. 2.1 a) als Querschnitt durch ein Doppelschichtpaket skizziert ist. Der Wasserstoff sollte sich in diesem Potential zunächst ausschließlich im Metall mit den 


\begin{tabular}{||c||c|c|c||c||}
\hline & $\begin{array}{c}D_{0} \\
{\left[\mathrm{~cm}^{2} / \mathrm{s}\right]}\end{array}$ & $\begin{array}{c}Q \\
{[\mathrm{~kJ} / \mathrm{mol}]}\end{array}$ & & $\begin{array}{c}D=D_{0} \cdot \exp -Q / R T \text { bei } 300 \mathrm{~K} \\
{\left[\mathrm{~cm}^{2} / \mathrm{s}\right]}\end{array}$ \\
\hline \hline $\mathbf{P d}$ & $5,25 \cdot 10^{-3}$ & 24,1 & {$[31]$} & $3,3 \cdot 10^{-7}$ \\
& $2,9 \cdot 10^{-3}$ & 22,2 & {$[33]$} & $4 \cdot 10^{-7}$ \\
\hline $\mathbf{V}$ & $3,5 \cdot 10^{-4}$ & 4,8 & {$[31]$} & $5,1 \cdot 10^{-5}$ \\
& $3,1 \cdot 10^{-4}$ & 4,3 & {$[33]$} & $5,4 \cdot 10^{-5}$ \\
\hline $\mathbf{N b}$ & $5,0 \cdot 10^{-4}$ & 10,2 & {$[31]$} & $8,2 \cdot 10^{-6}$ \\
& $5,0 \cdot 10^{-4}$ & 10,2 & {$[33]$} & $8,3 \cdot 10^{-6}$ \\
\hline $\mathbf{F e}$ & $9,3 \cdot 10^{-4}$ & 11,3 & {$[31]$} & $1 \cdot 10^{-5}$ \\
& $4.2 \cdot 10^{-4}$ & 6,1 & {$[33]$} & $4 \cdot 10^{-5}$ \\
\hline
\end{tabular}

Tabelle 2.2: $D_{0}$, Aktivierungsenergie $Q$ und daraus berechnete H-Diffusionskoeffizienten bei $300 \mathrm{~K}$.

energetisch tieferen Plätzen lösen (blau). Auch der H-Transport senkrecht zu den Schichten wird zu einem komplexen Diffusionsproblem, denn neben der unterschiedlichen Diffusion innerhalb verschiedener Schichten müssen noch die unterschiedlichen Löslichkeiten an den Grenzflächen berücksichtigt werden.

In realen Schichtsystemen kommen noch Abweichungen vom idealen periodischen Gitter speziell an den Grenzflächen hinzu. So sollten sich z.B. die von Hjörvarsson vorgeschlagenen elektronischen Effekte [16], eine Durchmischungszone oder die Existenz von Versetzungen zum Ausgleich der Gitterfehlpassung an der Grenzfläche auch in der Platzenergieverteilung niederschlagen. Abb. 2.1 b) verdeutlicht schematisch den möglichen Verlauf der Platzenergien bei elektronischen Übergangsstrukturen an der Grenzfläche. c) stellt den Verlauf bei einer Durchmischung der Grenzfläche dar. An den Grenzflächen liegende Plätze werden in beiden Fällen für den Wasserstoff energetisch ungünstiger, was in diesem Bereich zu einer Verarmung führt. Anhand des H-Signals läßt sich dieser Durchmischungseffekt nicht von einem elektronischen Effekt unterscheiden. Werden jedoch neben dem H-Signal auch die Metallkonzentrationen bestimmt, wie es Ziel dieser Arbeit ist, so kann eine H-Verarmung als Folge der Durchmischung detektiert werden.

In Abb. 2.1 d) ist als viertes Beispiel skizziert, daß Versetzungen an den Grenzflächen tiefere Platzenergien hervorrufen können. Als Platzenergieverteilung ist hier die von Kirchheim vorgeschlagene Verteilung an einer Stufenversetzung [29] eingezeichnet. Das Dilatationsfeld der Stufenversetzung liegt im eingezeichneten Fall in der höher lösenden Schicht. Der Wasserstoff wird bevorzugt an den Versetzungen gebunden. Die H-Löslichkeit wäre an der Grenzfläche erhöht. Für den H-Transport würde dieser Fall zu einer Verlangsamung senkrecht zu den Schichten führen, wohingegen die laterale Diffusion entlang der Grenzflächen beschleunigt sein könnte. 
a)

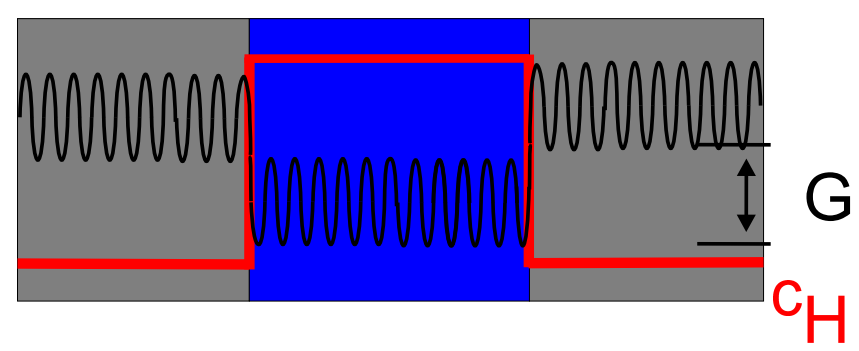

b)

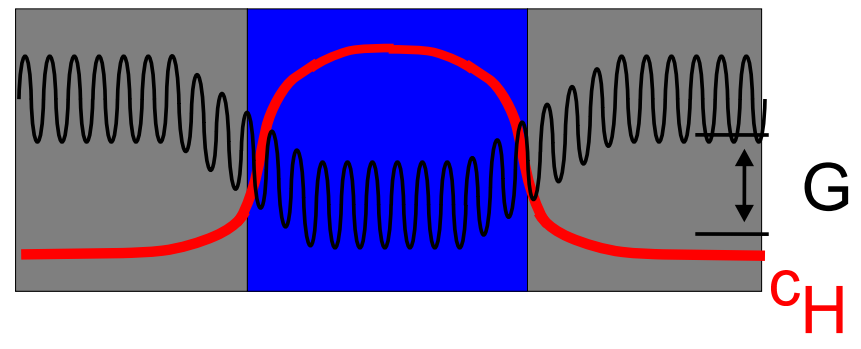

c)

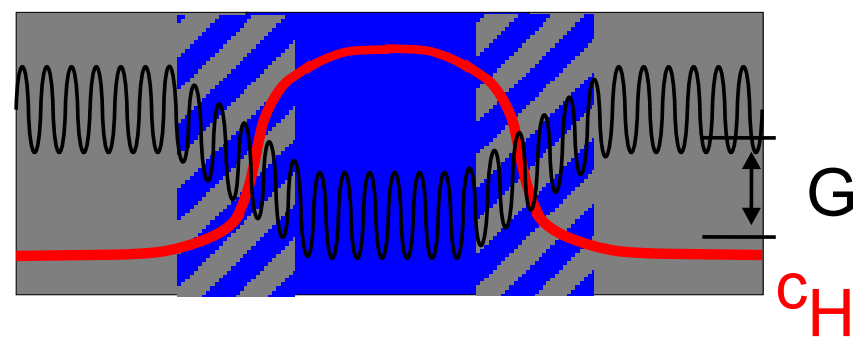

d)

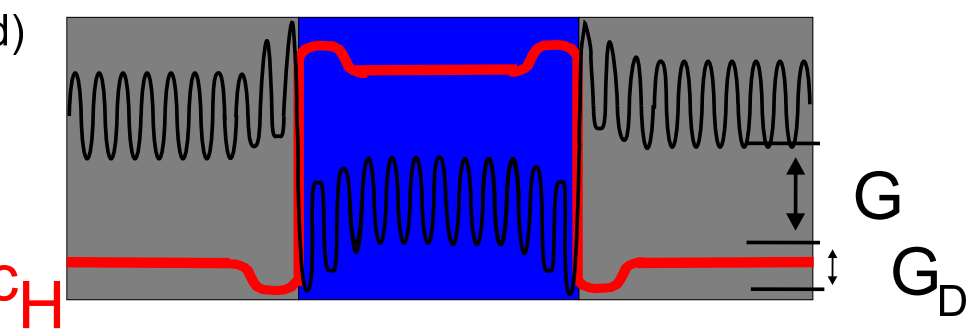

Abbildung 2.1: Schematische Verläufe der Platzenegieverteilung in Multischichtsystemen. Die blau gezeichnete Schicht besitzt die höhere H-Löslichkeit. Rot eingezeichnet ist der resultiernde H-Konzentrationsverlauf durch das Schichtpaket. a) ungestörtes periodisches Potential, b) Potentialveränderung an der Grenzfläche durch elektronische Effekte, c) Grenzflächenunschärfe durch chemische Durchmischung und d) Potentialabsenkung an der Grenzfläche z.B. durch den Einbau von Versetzungen.

\subsection{Verwendete Metall-H-Systeme}

Die untersuchten Multischichtsysteme wurden mit Metallen hergestellt, deren Metall-H-Systeme für die massiven Metalle gut untersucht sind und über die auch Ergebnisse für Dünnschichtsysteme bekannt sind. Die verwendeten Metalle und ihre zugehörigen Metall-H-Systeme sollen im folgenden Abschnit vorgestellt 
werden.

\subsubsection{Untersuchte Metallgrenzflächen}

Es wurden metallische Multischichtsysteme mit folgenden Grenzflächen hergestellt: $\mathrm{Nb} / \mathrm{Pd}, \mathrm{Nb} / \mathrm{W}, \mathrm{V} / \mathrm{Fe}$ und V/Pd. Dabei handelt es sich bis auf das $\mathrm{Pd}$ jeweils um kubisch raumzentrierte (krz) Metalle, Pd ist kubisch flächenzentriert (kfz). Für die strukturellen Eigenschaften der Grenzflächen ist dabei die Gitterfehlpassung zwischen den angrenzenden Metallen von Bedeutung. So führt eine hohe Gitterfehlpassung zu hohen Spannungen an der Grenzfläche, die durch den Einbau von Versetzungen an der Grenzfläche abgebaut werden können. Eine Zusammenstellung der Gitterparameter zeigt Tabelle 2.3 auf der Hauptdiagonalen der Tabelle. Die restlichen Tabellenwerte geben die jeweilige Gitterfehlpassung der untersuchten Metallgrenzflächen in der Kontaktebene an. Dabei werden die typischerweise gefundenen Wachstumsorientierungen senkrecht zu den Schichten in Dünnschichtsystemen von (110) für krz-Metalle und (111) für das kfz-Gitter des Pd [7] angenommen. Beim Aufwachsen eines krz- auf ein kfz-Gitter müssen sich in der Kontaktebene der [100]- und der $\left[\frac{1}{2} \frac{1}{2} \frac{1}{2}\right]$-Vektor im krz-Metall an die zwei $\left[\frac{1}{2} \frac{1}{2} 0\right]$-Vektoren im Pd anpassen. Das führt zu zwei Werten für die Gitterfehlpassung in der Tabelle beim Pd. Man erkennt, daß jeweils eine Richtung in der Kontaktebene für die Nb/Pd- und V/Pd-Grenzfläche eine sehr große Gitterfehlpassung von $20 \%$ bzw. 10\% aufweist. In diesen Grenzflächen kann somit eine hohe Dichte an Anpassungsversetzungen erwartet werden. Diese treten jedoch erst oberhalb einer kritischen Schichtdicke auf [38].

\begin{tabular}{|c|c|c|c|c|c|}
\hline $\mathrm{a}[\mathrm{nm}]$ & $\mathbf{V}$ & $\mathrm{Nb}$ & $\mathrm{Fe}$ & $\mathbf{W}$ & Pd \\
\hline \multirow{5}{*}{$\begin{array}{c}\mathrm{V} \\
\mathrm{Nb} \\
\mathrm{Fe} \\
\mathrm{W} \\
\mathrm{Pd}\end{array}$} & 0,30240 & & & & \\
\hline & \multirow{3}{*}{0,055} & 0,33000 & & & \\
\hline & & \multirow{3}{*}{$\begin{array}{c}0,043 \\
0,200 / 0,040\end{array}$} & 0,28664 & & \\
\hline & & & & 0,31650 & \\
\hline & $0,100 /-0,047$ & & & & 0,38870 \\
\hline
\end{tabular}

Tabelle 2.3: Auf der Hauptdiagonalen aufgetragen sind die Gitterkonstanten der verwendeten Metalle im massiven Material. Die anderen Werte der Tabelle geben die Gitterfehlpassung der untersuchten Metallgrenzflächen auf Basis der angegeben Gitterkonstanten an. Details der Orientierungsbeziehung zwischen krzund kfz-Gitter finden sich im Text.

Den Werten der Tabelle 2.3 zu Folge sollten besonders V/Fe und Nb/W mit hoher Kohärenz, d.h. einer geringen Versetzungsdichte zum Ausgleich der Gitterfehlpassung, aufeinander aufwachsen. So gelingt es der Gruppe von Hjörvarsson V/Fe-Schichtpakete mit 15 Doppelschichten und Einzelschichtdicken von $2 \mathrm{~nm} \mathrm{~V}$ 


\begin{tabular}{||c|c||}
\hline & $\begin{array}{c}\mathrm{k} \text { bei } 300 \mathrm{~K} \\
\left(=\mathrm{c}_{M e_{1}} / \mathrm{c}_{M e_{2}}\right)\end{array}$ \\
\hline \hline $\mathbf{P d} / \mathbf{N b}$ & $3 \cdot 10^{-5}$ \\
$\mathbf{W} / \mathbf{N b}$ & $8 \cdot 10^{-24}$ \\
$\mathbf{P d} / \mathbf{V}$ & $5 \cdot 10^{-4}$ \\
$\mathbf{F e} / \mathbf{V}$ & $5 \cdot 10^{-10}$ \\
\hline
\end{tabular}

Tabelle 2.4: H-Löslichkeitsunterschiede $k$ für die untersuchten Metallgrenzfl̈̈chen, berechnet mit den Werten aus Tab. 2.1.

und $3 \mathrm{~nm}$ Fe in Übergitterstrukturen herzustellen [39], bei denen Fe und V in einem gemeinsamen Gitter mit einem mittleren Gitterparameter von 0,2915 nm in der Schichtebene aufwachsen. Die Gitterfehlpassung in der Ebene beträgt somit $-3,8 \%$ im V und $+1,7 \%$ im Fe. In Richtung der Schichtnormalen ermitteln sie für die periodisch aufeinander aufgewachsenen Schichten einen Gitterparameter von 0,3053 nm im V und 0,2830 $\mathrm{nm}$ im Fe. Die Schichten sind demnach tetragonal verzerrt. Die Autoren berichten von einer Kohärenzlänge in der Kontaktebene von 50 nm. Bei Metallgrenzflächen mit höherer Gitterfehlpassung und größeren Schichtdicken sollte diese Kohärenzlange entsprechend kleiner werden. Unterschiede in der Kohärenz der Grenzflächen sollten durch ihren Einfluß auf die Zwischengitterplatzenergien an der Grenzfläche auch im Löslichkeits- und Diffusionsverhalten des Wasserstoffs eine Rolle spielen. Das gleiche gilt für die chemische Schärfe der Grenzfläche.

\subsubsection{Phasendiagramme der Metall-H-Systeme}

Die Zusammensetzung der Multischichten wurde so gewählt, daß man jeweils ein Metall mit hoher H-Löslichkeit mit einem geringer Löslichkeit kombiniert. Dadurch sollte ein auftretender Grenzflächeneffekt der H-Löslichkeit durch die Veränderung der elektronischen Struktur an den Grenzflächen deutlich zu Tage treten. So wurden $\mathrm{Nb}$ und $\mathrm{V}$ als hochlösende Komponenten gewählt und mit Pd, Fe und W kombiniert. Der Tabelle 2.1 läßt sich der Löslichkeitsunterschied bezogen auf das Pd entnehmen. Die daraus folgenden Löslichkeitsunterschiede für die untersuchten Metallgrenzflächen sind in Tabelle 2.4 angegeben. Die größten Löslichkeitunterschiede findet man somit an der Nb/W- und Fe/V-Grenzfläche. Die Kombinationen von $\mathrm{Pd}$ mit $\mathrm{Nb}$ und $\mathrm{V}$ zeigen dagegen deutlich geringere Unterschiede.

Die ausgewählten Metalle lassen sich in zwei Kategorien aufteilen. Bei den Metallen $\mathrm{V}$ und $\mathrm{Nb}$ handelt es sich um Systeme, die den Wasserstoff exotherm lö sen, bei denen sich der Wasserstoff bei Raumtemperatur unter Energiegewinnung lösen läßt. Ausgedrückt wird dies durch das negative Vorzeichen in der 
Freien Lösungsenergie (s. Tab. 2.1). Pd ist hier ein Grenzfall, da bei $300 \mathrm{~K}$ die Enthalpie schon positiv wird. In diesen Systemen findet man in massiven Metallen eine hohe Randlöslichkeit des Wasserstoffs bei Raumtemperatur, die von $1 \%$ im Pd bis zu $6 \%$ im Nb reicht. Die Phasendiagramme des V- und Nb-HSystems sind in den Abbildungen 2.2 und 2.3 dargestellt. $\mathrm{V}$ und $\mathrm{Nb}$ sind beide Übergangsmetalle der V. Nebengruppe und besitzen ein ähnliches Lösungsverhalten für den Wasserstoff. Die Mischungslücke zwischen $\alpha$ - und $\beta$-Phase erstreckt sich beim V-H-System bis zu Konzentrationen von $0,5 \mathrm{H} / \mathrm{V}$ und beim $\mathrm{Nb}$ bis 0,7 $\mathrm{H} / \mathrm{Nb}$. Für höhere Konzentrationen wurden eine Vielzahl unterschiedlicher Hydridphasen nachgewiesen. Im Rahmen dieser Arbeit möchte ich mich jedoch auf die Beschreibung der $\alpha$ - und $\beta$-Phasen beschränken. Eine detaillierte Behandlung der weiteren Phasen ist in [35] zu finden. Auch beschränke ich mich hier auf die Angabe des Metall-H-Diagramms. Zwar kommt es in den Metall-D-Systemen zu einigen Abweichungen [35], jedoch sind diese im Vergleich zu den Veränderungen des Phasendiagramms, die man in Dünnschichtsystemen beobachtet, vernachlässigbar.

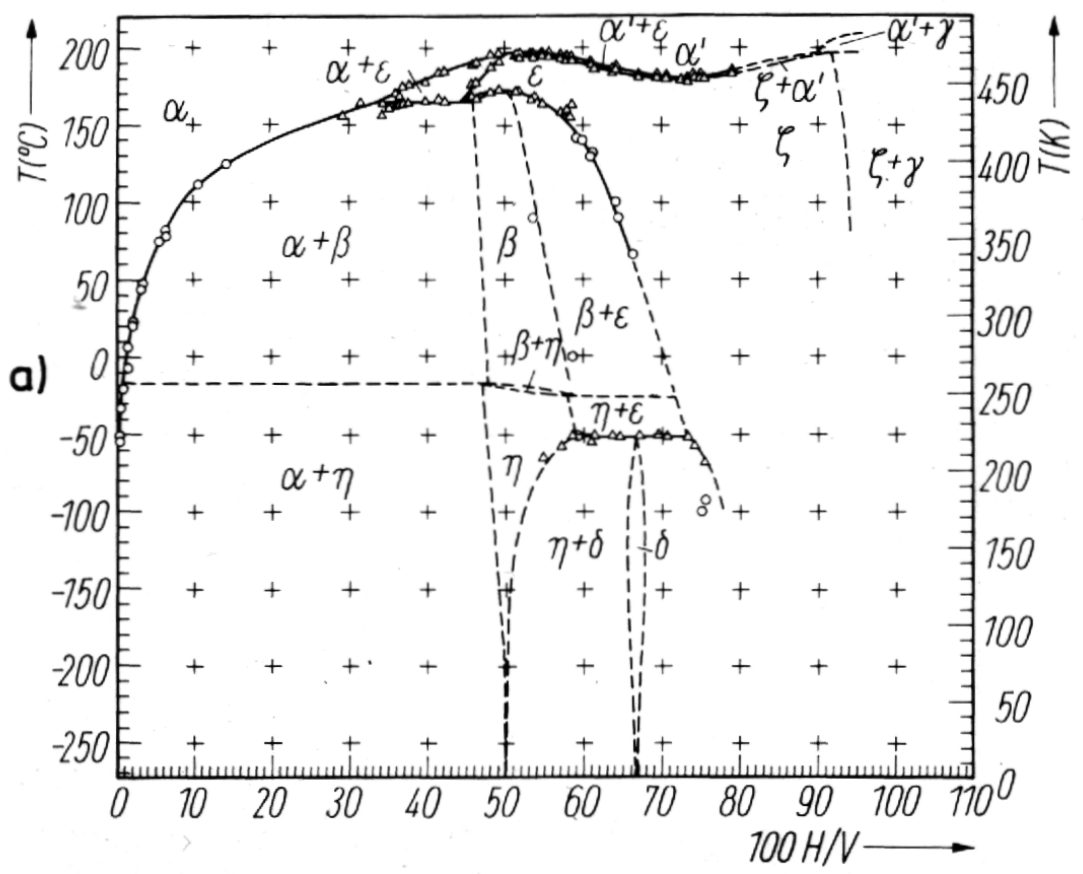

Abbildung 2.2: V-H-Phasendiagramm [35].

In Fe und W läßt sich Wasserstoff dagegen nur endotherm lösen. Die entsprechenden Metall-H-Systeme zeigen demzufolge bei Raumtemperatur nur eine verschwindend geringe Randlöslichkeit. In der Kombination mit den hochlösenden Metallen sollte somit näherungsweise kein Wasserstoff in Fe und W gelöst werden. Aus diesem Grund sind die entsprechenden Phasendiagramme hier nicht 


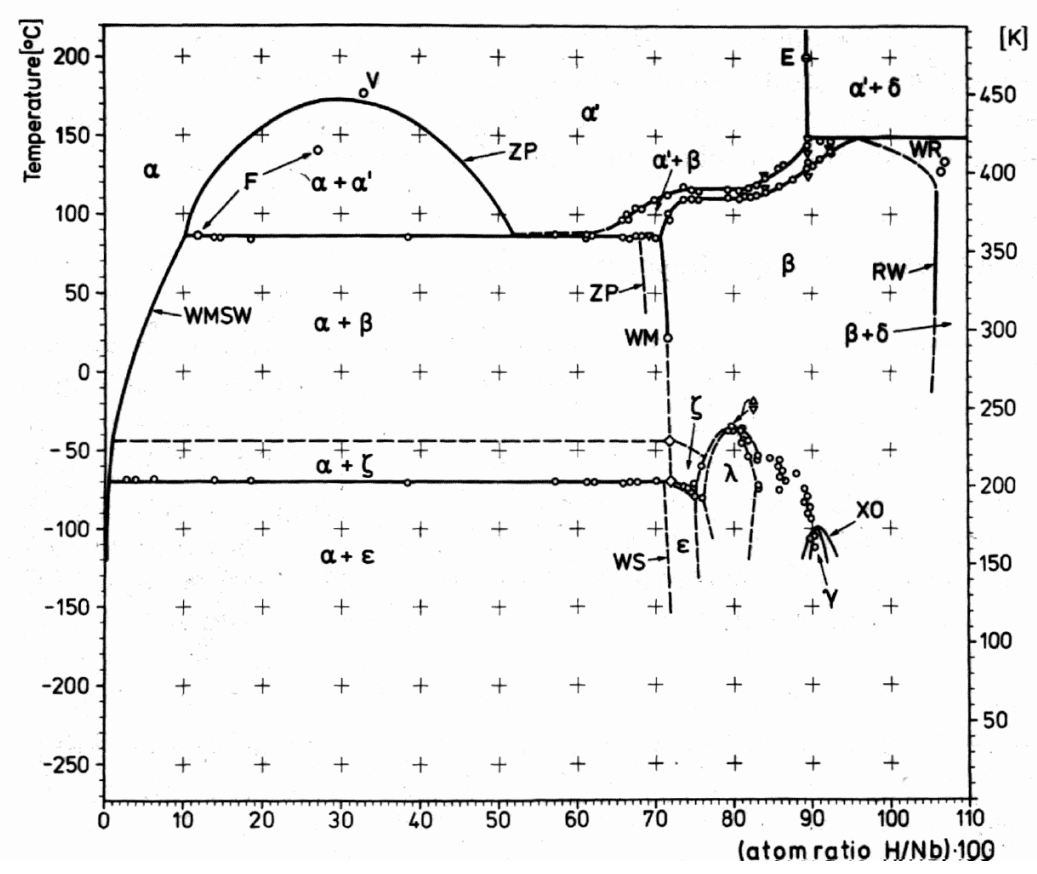

Abbildung 2.3: Nb-H-Phasendiagramm [35].

aufgeführt, man findet sie in [40].

Um die Mischungstendenz der untersuchten Metallkombinationen beurteilen zu können, werden im folgenden Abschnitt die Phasendiagramme der betreffenden binären Systeme dargestellt.

\subsubsection{Binäre Phasendiagramme der Metallsysteme}

Beim System Nb-W handelt es sich um ein lückenlos mischbares System. Im mischenden System Fe-V zeigt sich unterhalb von $1200^{\circ} \mathrm{C}$ eine breite intermetallische $\sigma$-Phase. Die Bildung der $\sigma$-Phase ist häufig kinetisch unterdrückt [41]. Die Systeme $\mathrm{Pd}-\mathrm{Nb}$ und $\mathrm{Pd}-\mathrm{V}$ sind mischend mit vielen stabilen intermetallischen Phasen und besitzen eine hohe Randlöslichkeit. So löst sich Pd in Nb bzw. V und umgekehrt Nb bzw. V in Pd mit einer Randlöslichkeit von ca. 20\% [40].

Diese Daten zeigen, daß die ausgewählten Metallsysteme zwar bezüglich der H-Löslichkeit optimiert sind, jedoch bei allen betrachteten Systemen im Gleichgewicht eine Mischungstendenz festzustellen ist. Es ist eine Frage der Kinetik und somit der Temperatur, ob und auf welchen Längenskalen diese Durchmischung stattfindet. Da die Temperatur bei der Herstellung und weiteren Behandlung der Proben je nach System nicht über $200-320^{\circ} \mathrm{C}$ lag, ist bei den verwendeten kubisch raumzentrierten Metallen nur mit einer sehr geringen Interdiffusion zu rechnen. Mit den angewendeten Analyseverfahren können eventuell auftretende Interdiffusionszonen auch detektiert werden. 


\section{Kapitel 3}

\section{Chemische Analysetechniken auf Nanometerskala}

Für direkte Untersuchungen der lokalen H-Verteilung in den zu untersuchenden Schichtsystemen benötigt man Analyseverfahren, die eine entsprechend hohe Auflösung bieten. Am Institut für Materialphysik stehen dabei mit dem Feldionenmikroskop (FIM) in Kombination mit einer tomographischen Atomsonde (TAP = tomographic atom probe) und einem Sekundärionenmassenspektrometer (SIMS) zwei Analysegeräte zur Verfügung, die es beide erlauben, elektrisch leitende Proben atomlagenweise abzutragen und dabei die chemische Zusammensetzung der Probe zu bestimmen. Auch wenn die Methoden durch systembedingte Fehler und die nötige Rekonstruktion der gewonnenen Daten diesen atomaren Abtrag nicht gänzlich auflösen können, gelingt es beim FIM/TAP, eine Auflösung im Subnanometerbereich zu erreichen und im SIMS zumindest in der Abtragsrichtung eine Auflösung im Nanometerbereich zu realisieren. Die Prinzipien dieser Analyseverfahren und dadurch bedingte Stärken und Schwächen sollen im folgenden erläutert werden.

\subsection{Feldionenmikroskop (FIM) und Tomographi- sche Atomsonde (TAP)}

\subsubsection{Feldionenmikroskop}

Das Feldionenmikroskop (FIM) erlaubt die Abbildung elektrisch leitfähiger Oberflächen durch Feldionisation in $10^{6}$ - bis $10^{7}$-facher Vergrößerung. Durch Feldverdampfung können die Proben zusätzlich atomlagenweise abgetragen werden. Eine schematische Skizze des FIM zeigt die linke Seite der Abbildung 3.1 (gestrichelter Kasten und Bildschirm in Position a)). Die nötigen Feldstärken von 10-50 $\frac{\mathrm{V}}{n m}$ erhält man durch Anlegen einer Hochspannung von bis zu $20 \mathrm{kV}$ an spitzenförmige Proben mit Krümmungsradien unterhalb von $100 \mathrm{~nm}$ im UHV bei Basisdrücken 
von einigen $10^{-10}$ mbar. Zur Abbildung der Oberfläche wird ein sogenanntes Bildgas (meist He und Ne) mit Drücken von $10^{-5}$ mbar in die Kammer eingelassen. Die Bildgasatome werden im zwischen Probe und Abbildungssystem angelegten Hochspannungsfeld polarisiert und an die Probenoberfläche gezogen. Dort werden sie feldionisiert und radial zum Bildschirm hin beschleunigt, wo die Einschläge der Bildgasionen einen stehenden Leuchtpunkt erzeugen. Durch eine bevorzugte Ionisierung an Gebieten hoher lokaler Krümmung, die vor allem an den Kantenatomen der Gitterebenen vorliegen, gelingt eine Abbildung der Kristalloberfläche der Probe. Zur Unterdrückung thermischer Ionisationsprozesse werden die Proben auf Temperaturen unterhalb von $150 \mathrm{~K}$ gekühlt. Die ausführliche Beschreibung der Methode und des Abbildungsprozesses findet man in der Literatur [42]. Das am Institut für Materialphysik installierte FIM ist in [43] beschrieben.

TAP:

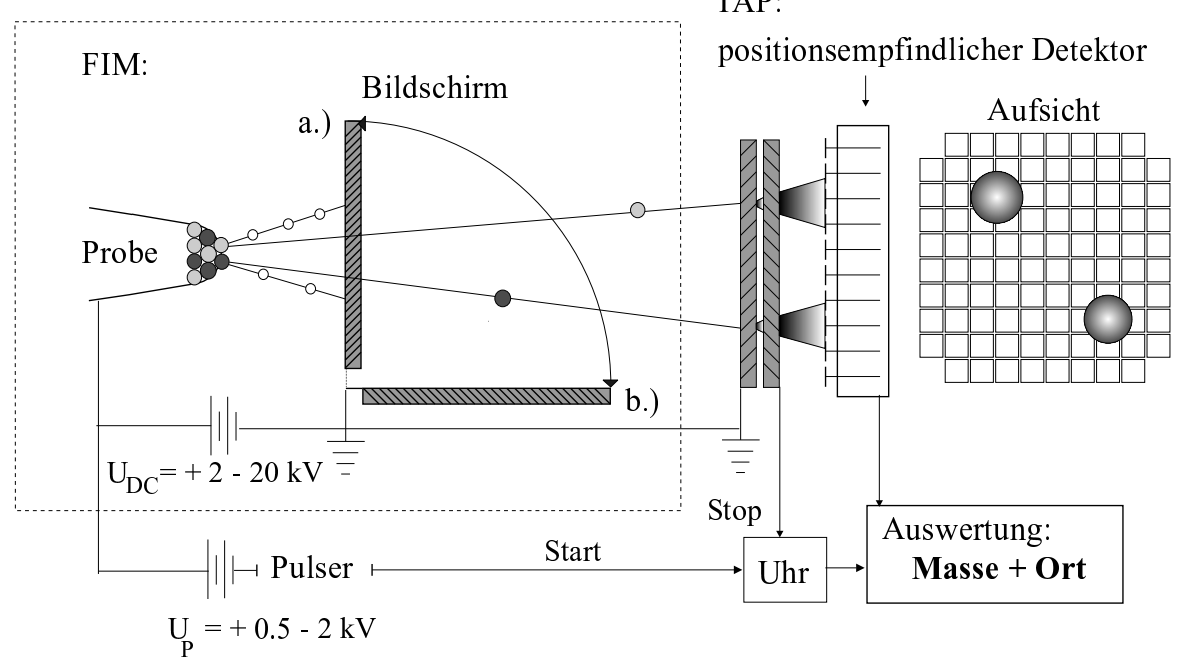

Abbildung 3.1: Prinzipskizze eines Feldionenmikroskopes mit tomographischer Atomsonde. Ist der Bildschirm in Position a) bildet man, wie im Text erläutert, die Probe ab. In Position b) läuft das Mikroskop im Atomsondenmodus, die Atome werden feldverdampft und zum Detektor hin beschleunigt. Im rechten Teil ist der positionsempfindliche Detektor und eine Aufsicht auf die Anoden zur Bestimmung des Ladungsschwerpunktes skizziert.

\subsubsection{Tomographische Atomsonde}

Für die chemische Analyse der präparierten Schichtsysteme wurde die tomographische Atomsonde (TAP) genutzt. Eine Skizze zeigt Abb. 3.1 (rechter Teil, Bildschirm in Position b)). Sie ist eine Weiterentwicklung der konventionellen Atomsonde ( $\mathrm{AP}=$ atom probe), eines Flugzeit-Massenspektrometers, mit dem man im FIM mittels Feldverdampfung einzelner Atome eine chemische Analyse der Pro- 
be durchführen kann. Dazu wird zusätzlich zur angelegten Grundspannung $U_{D C}$ ein Spannungspuls $U_{P}$ angelegt, so daß die Feldverdampfungsfeldstärke des Probenmaterials überschritten wird und einzelne Atome aus der Probenoberfläche feldionisiert und in Richtung des Detektors beschleunigt werden. Der Puls dient gleichzeitig als Startsignal einer Uhr, die durch das Auftreffen der Ionen auf dem Detektor gestoppt wird. Mit der so erhaltenen Flugzeit kann bei bekannter Fluglänge und Spannung auf das $\frac{e}{m}$-Verhältnis des auftreffenden Ions und somit auf seine Masse geschlossen werden. Eine ausführliche Beschreibung des AP-Prinzips findet sich in $[44,45]$, die im Institut für Materialphysik installierte AS ist in [43] beschrieben.

Mit konventionellen Atomsonden war bisher keine laterale Rekonstruktion der detektierten Ionen aus dem abgetragenen Oberflächenbereich von typischerweise $100 \mathrm{~nm}^{2}$ möglich. Die an der Universität Rouen entwickelte [46, 47] und auch in Göttingen installierte tomographische Atomsonde ermöglicht mit Hilfe eines positionsempfindlichen Detektors eine solche Oberflächenrekonstruktion. Die Position der auftreffenden Ionen wird auf einem Anodenfeld (s. Abb. 3.1) über die Bestimmung des Ladungsschwerpunktes ermittelt. Einen Überblick über die physikalischen Grundlagen tomographischer Atomsonden, ihre technische Umsetzung, die Datenrekonstruktion und -analyse und über bisherige Anwendungen dieser jungen Methode geben Al Kassab et al. [48].

Die Analyse der Proben mit der tomographischen Atomsonde wird im UHV durchgeführt, um die Anzahl der detektierten Ionen aus dem Restgas möglichst gering zu halten.

Die Meßergebnisse der TAP-Analysen bestehen aus Rohdaten, die für jedes detektierte Ion u.a. die Flugzeit $t$, den Auftreffort $\left(x_{D}, y_{D}\right)$, die Grundspannung $U_{D C}$ und die Pulsspannung $U_{P}$ enthalten. Die dreidimensionale Rekonstruktion der detektierten Ionen ist ausführlich in [48] beschrieben, die grundlegende Vorgehensweise soll im folgenden skizziert werden.

- Aus der Flugzeit $t$ berechnet man mit der Energieerhaltung einer im elektrischen Feld beschleunigten Masse $m$ und der Fluglänge $L$ die spezifische Masse $\frac{m}{n}$, mit dem Ionisierungsgrad $n$.

$$
\begin{aligned}
& n e U=\frac{1}{2} m v^{2}=\frac{1}{2} m \frac{L^{2}}{t^{2}} \\
& \frac{m}{n}=\frac{2 e \cdot\left(U_{D C}+U_{P}\right) t^{2}}{L^{2}+x_{D}^{2}+y_{D}^{2}}
\end{aligned}
$$

- Der Auftreffort wird auf die laterale Position auf der Spitzenoberfläche zurückgerechnet. Dabei bedient man sich einfacher Punktprojektionen und geht von einem kugelförmigen Spitzenapex aus, der in einen Schaft mit konstantem Winkel übergeht. 
- Der Abtrag in z-Richtung wird so berücksichtigt, daß pro Atom ein zInterval angesetzt wird, das u.a. mit dem Atomvolumen, der Detektionswahrscheinlichkeit, der Detektorfläche und dem Spitzenradius skaliert.

Der Spitzenradius $R$ wird im allgemeinen aus dem Quotienten aus angelegter Spannung $U_{D C}+U_{P}$ und der materialspezifischen Feldverdampfungsfeldstärke $E$ berechnet, korrigiert mit einem Geometriefaktor $\beta$, der typischerweise zwischen 5 und 10 liegt und die Abweichung von der idealen Kugelform berücksichtigt [48]

$$
R=\frac{U_{D C}+U_{P}}{E \cdot \beta}
$$

In Multischichtsystemen, bei denen die Feldverdampfungsfeldstärke über die Messung nicht konstant ist, führt dieser Ansatz jedoch zu unterschiedlichen Volumenrekonstruktionen in Gebieten unterschiedlicher Feldverdampfungsfeldstärken. Eine geometrische Bestimmung des Spitzenradius ist in solchen Systemen vorzuziehen. Deshalb wurde in den Auswertungen der von G.Schmitz entwickelte Rekonstruktionsalgorithmus [48], in den als geometrische Parameter der Anfangsradius und der Schaftwinkel der Spitze eingehen, verwendet. Er liefert als Ergebnis auch das Produkt $E \cdot \beta$ als Funktion der rekonstruierten Tiefe. Durch einen Vergleich von $E \cdot \beta$ mit Referenzmessungen kann die Qualität der Rekonstruktion überprüft werden. Die Wahl der geometrischen Parameter bei der Rekonstruktion der gemessenen Schichtsysteme wurde so durchgeführt, daß

- die aus Transmissionselektronenmikroskopie gewonnene Geometrie und die Schichtdicken der Spitze wiedergegeben werden,

- die Fläche des rekonstruierten Volumens mit der durch den Detektor meßbaren Fläche übereinstimmt, die je nach angelegter Spannung zwischen $8 \mathrm{x}$ $8 \mathrm{~nm}^{2}$ und $15 \times 15 \mathrm{~nm}^{2}$ liegt,

- vorhandene Gitternetzebenen möglichst eben dargestellt werden und

- vorhandene Gitternetzebenen möglichst mit dem in der Literatur angegebenen Netzebenenabstand dargestellt werden.

Die rekonstruierten Daten lassen sich in vielfältiger Weise analysieren. Mit 3DVisualisierungsprogrammen (verwendet wurde AVS) lassen sich die gemessenen Volumina darstellen, indem jedes einem ausgewähltem Massenfenster zugeordnete Atom als Kugel angezeigt wird. Durch die Auswahl der Massenfenster lassen sich so je nach physikalischer Fragestellung die Verteilungen einzelner oder mehrerer Elemente in der Probe darstellen. Abbildung 3.2 zeigt die nach den oben angegebenen Punkten durchgeführte Rekonstruktion der Messung einer W-Probe. Das rekonstruierte Volumen beträgt $10 \times 10 \times 8 \mathrm{~nm}^{3}$. Jeder Punkt entspricht einem detektierten Atom aus dem W-Massenfenster. Man erkennt die (110)-Ebenen, die senkrecht zur Meßrichtung angeordnet sind. Schaftwinkel und Anfangsradius der 
Rekonstruktion wurden so gewählt, daß der Ebenenabstand $d=\frac{a}{\sqrt{2}}=2,23$ korrekt wiedergegeben wird (s.Tab. 2.3). Als Atomvolumen wurde der Literaturwert von $15,8^{3}$ eingesetzt. Das Produkt $E \cdot \beta$ nimmt dann den Wert $25 \underline{V}$ an. Für

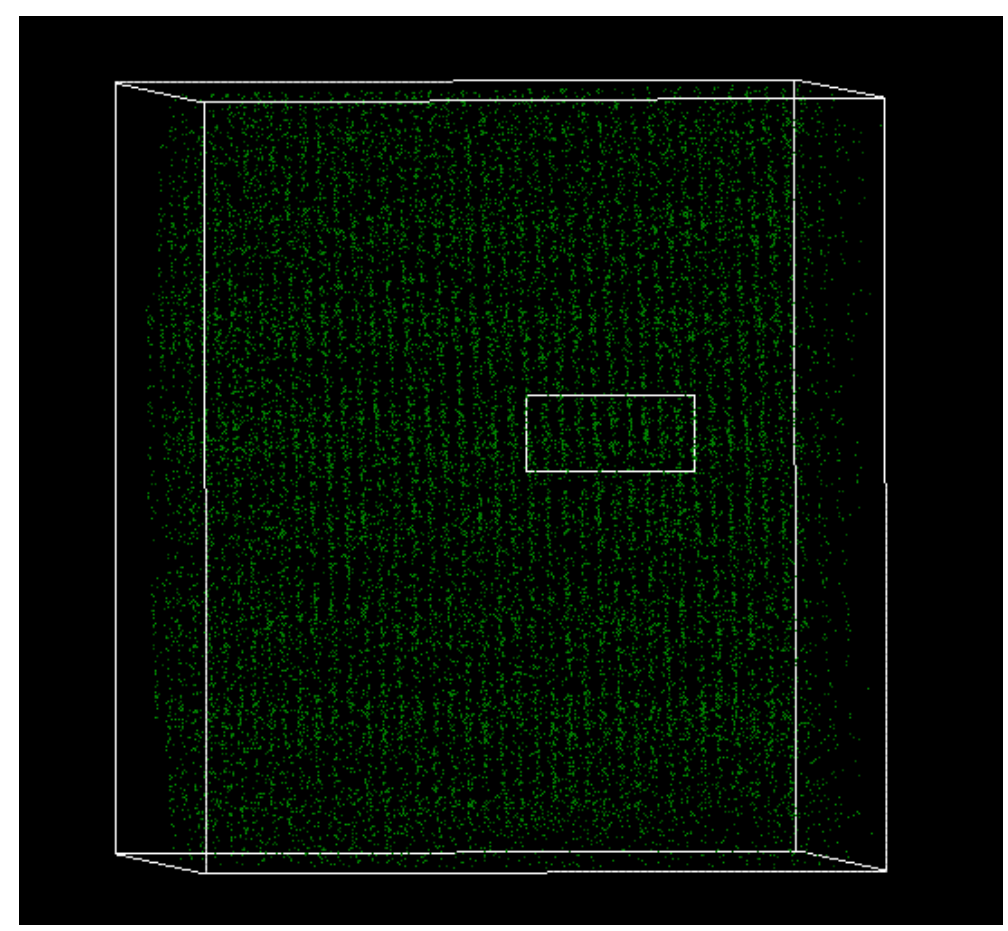

Abbildung 3.2: TAP-Analyse einer W-Probe, gemessen bei $60 \mathrm{~K}$. Das Volumen beträgt $10 \times 10 \times 8 \mathrm{~nm}^{3}$ und wurde mit dem Atomvolumen von $W\left(15,8^{3}\right)$, einem Anfangsradius von $310 \mathrm{~nm}$ und einem Schaftwinkel der Spitze von $3 \mathrm{~nm}$ rekonstruiert. Im kleinen Rahmen der Länge 2,23 $\mathrm{nm}$ findet man zehn (110)Ebenen.

die untersuchten Systeme sind in Tabelle 3.1 mittlere Werte der Rekonstruktionen für $E \cdot \beta$ angegeben. Aufgeführt sind zusätzlich in der Literatur angegebene Werte für die Verdampfungsfeldstärke $E$, die jedoch aus einfachen geometrischen Modellen berechnet wurden. Vergleicht man das gemessene Produkt $E \cdot \beta$ mit den Literaturwerten für $E$, so erhält man den Geometriefaktor $\beta$, der zwischen 5 und 10 liegen sollte. Wie Tab. $3.1 \mathrm{zu}$ entnehmen, ist dies bei den durchgeführten Rekonstruktionen der Fall.

Neben der direkten Abbildung der gemessenen Atome kann durch die Analyse einzelner Teilvolumina der Probe die lokale Konzentration der gemessenen Atome bestimmt werden. Dabei führt ein möglichst kleines Volumen zwar zu einer hohen räumlichen Auflösung, jedoch steigt der Fehler der Konzentrationsbestimmung mit abnehmender Anzahl der Atome im analysierten Volumen. Für eine binäre Legierung kann der Fehler bei der Bestimmung der Konzentration $c$ in einem 


\begin{tabular}{|c|c|c|c|c|}
\hline System & $V_{A}\left[{ }^{3}\right]$ & $E \cdot \beta[V / A]$ & $E_{L i t}[V / A][44]$ & $\beta$ \\
\hline $\mathrm{W}$ & 15.8 & $25-30$ & 5,2 & $5-6$ \\
$\mathrm{Pd}$ & 14.7 & 24 & 3,7 & 6 \\
$\mathrm{Nb}$ & 18.1 & 25 & 3,7 & 7 \\
$\mathrm{Fe}$ & 11.8 & $20-22$ & 3,3 & $6-7$ \\
$\mathrm{~V}$ & 14.6 & $21-23$ & 3,0 & $7-8$ \\
\hline
\end{tabular}

Tabelle 3.1: Atomvolumina und mittlere Werte für $E \cdot \beta$ bei der Rekonstruktion der TAP-Daten.

Volumen mit $N$ Atomen wie folgt abgeschätzt werden:

$$
\Delta c \approx \sqrt{\frac{c(1-c)}{N}} .
$$

Bei den, in Kapitel 5 beschriebenen, an den Multischichten durchgeführten Konzentrationsanalysen ist demzufolge jeweils die Größe des Analysevolumens und die Atomanzahl für die Bestimmung eines Konzentrationswertes angegeben. Ein großer Vorteil der Methode ist, daß das Analysevolumen frei wählbar ist. Dadurch kann z.B. im Fall der zu untersuchenden Grenzfächen die Konzentrationsanalyse stets senkrecht zu den Grenzflächen durchgeführt werden.

\subsection{Sekundärionenmassenspektrometer (SIMS)}

Mit dem TOF-SIMS IV steht eine weitere time of flight(TOF)-Atomsonde zur chemischen Analyse von Schichtsystemen zur Verfügung. Hier werden die Atome durch den Einschlag von Primärionen und dadurch in der Schicht ausgelöste Stoßkaskaden aus der Probe herausgeschlagen. Aus der Probe geschlagene Teilchen können dann im ionisierten Zustand als sogenannte Sekundärionen mit dem gleichen Prinzip wie im FIM/TAP auf ihr e/m Verhältnis analysiert weren. In dem man den Primärionenstrahl gepulst auf die Probe schickt, erhält man die benötigte Startzeit als Pulsbeginn und stoppt die Flugzeit beim Auftreffen auf den Analysator. Da der Pulsbeginn vor dem tatsächlichen Austreten der Ionen aus der Probe liegt, ist die gemessene Flugzeit zu lang, weicht aber von der tatsächlichen Flugzeit der herausgeschlagenen Ionen stets nur um eine Konstante ab, so daß man durch Verschieben des Spektrums auf die tatsächlichen Massen kommt. Als Primärionen werden Ga-Ionen mit Energien von 11-25 keV verwendet. Eine schematische Zeichnung der SIMS-Apparatur zeigt Abb. 3.3.

Ein bedeutender Vorteil des SIMS gegenüber dem FIM/TAP ist die Möglichkeit, planare Schichtsysteme untersuchen zu können. Zudem ist das analysierte Volumen größer. Zwar werden auch nur Ionen aus der Oberfläche und einigen Atomlagen Tiefe herausgelöst, doch regt man mit dem fokussierten GaIonenstrahl stets Flächen von mindestens $0,1 \times 0,1 \mu m^{2}$ an. Lateral handelt es sich 


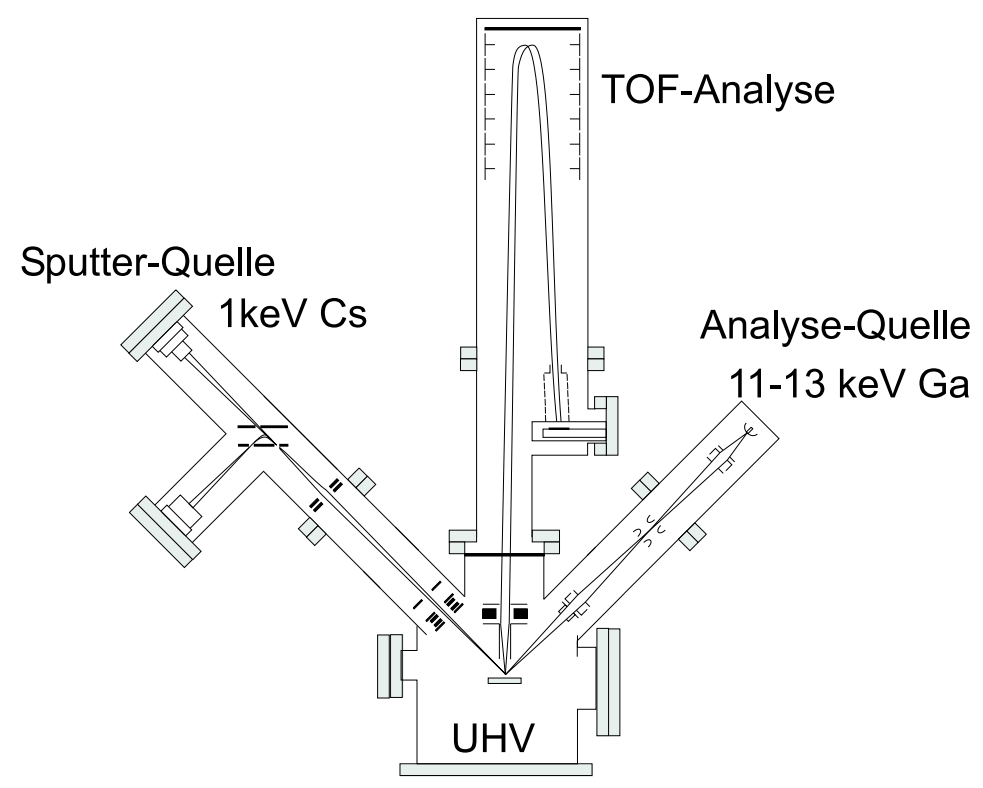

Abbildung 3.3: Schematischer Aufbau des TOF-SIMS.

somit um keine nanoskalige Analysemethode. Durch Umlenkung des Ga-Strahls ist es möglich, eine Fläche von bis zu 500 x $500 \mu m^{2}$ abzurastern und dadurch laterale Verteilungen in dieser Größenordnung aufzunehmen. Da die Stoßprozesse nur einzelne Atomlagen beeinflussen, und man dementsprechend nur einen vernachlässigbaren Tiefenabtrag erhält, ist das SIMS zusätzlich mit einer zweiten Ionenquelle ausgestattet, die es erlaubt, großflächig, auf einer Fläche von bis zu $500 \times 500 \mu \mathrm{m}^{2}$, Material abzusputtern und somit einen nennenswerten Tiefenabtrag zu erreichen. Verwendet werden Ar- oder Cs-Ionen mit Energien von 1-5 $\mathrm{keV}$. Die Analyse erfolgt dann durch den abwechselnden Einsatz von Analyseund Sputter-Ionenstrahl. Außerdem kann die Analyse bei $300 \mathrm{~K}$ durchgeführt werden. Da eine Kühlung der Proben am Göttinger Gerät nicht möglich ist, wurden alle Messungen bei $300 \mathrm{~K}$ durchgeführt. Zur Statistik des Detektionsprozesses ist anzumerken, daß nur ein Bruchteil von einigen Prozent der abgetragenden Atome detektiert werden, so daß typischerweise 20000 Ionen pro Sekunde gemessen werden.

Nachteilig oder zumindest schwer zu analysieren sind jedoch die Effekte der auftreffenden Ionenstrahlen, die für eine Einlagerung der Ionenelemente in die Probe und für eine Durchmischung der untersuchten Schichten sorgen. Diese Effekte müssen für eine quantitative Analyse an entsprechenden Standardproben kalibriert werden, können auf der anderen Seite jedoch auch zu erhöhten Ionenausbeuten und dadurch verbesserter Statistik führen. Im Falle des Cäsiums führen die eingebrachten Atome zur Bildung von Metallcäsiden, die nahezu 
unabhängig von der Zusammensetzung der Probe ionisiert werden, so daß sich die detektierten Metallcäside in einigen Systemen quantitativ bezüglich der chemischen Zusammensetzung der Probe auswerten lassen. In [49, 50, 51] konnten quantitative Analysen von Metallcäsidsystemen durch die Verwendung eines 5-10 keV-Cs-Sputter-Ionenstrahls demonstriert werden. Für die Analyse von Pd/NbSchichtsystemen und deren Hydriden wurde eine Kombination von Ga-Analyseund Cs-Sputter-Ionenstrahl gewählt, die gut reproduzierbare und quantitativ analysierbare Daten erzeugt. Die Messungen wurden bei nahezu konstanten Energien der Sputter-Quelle (1kV Cs-Ionen) und Analyse-Quelle (11-12,5 kV Ga-Ionen) durchgeführt. Die Ionenströme varieren geringfügig durch die nicht völlig reproduzierbare Justage der Quellen. Typische Ströme waren (4士1) nA für die Cs-Quelle und $(1,5 \pm 0,5) \mathrm{pA}$ für die Ga-Quelle. Die Cs-Quelle wurde mit $1 \mathrm{keV}$ statt 5 $\mathrm{keV}$ betrieben, da sich an den untersuchten Multischichtsystemen bei höheren Cs-Energien eine starke Durchmischung an den Grenzflächen zeigte.

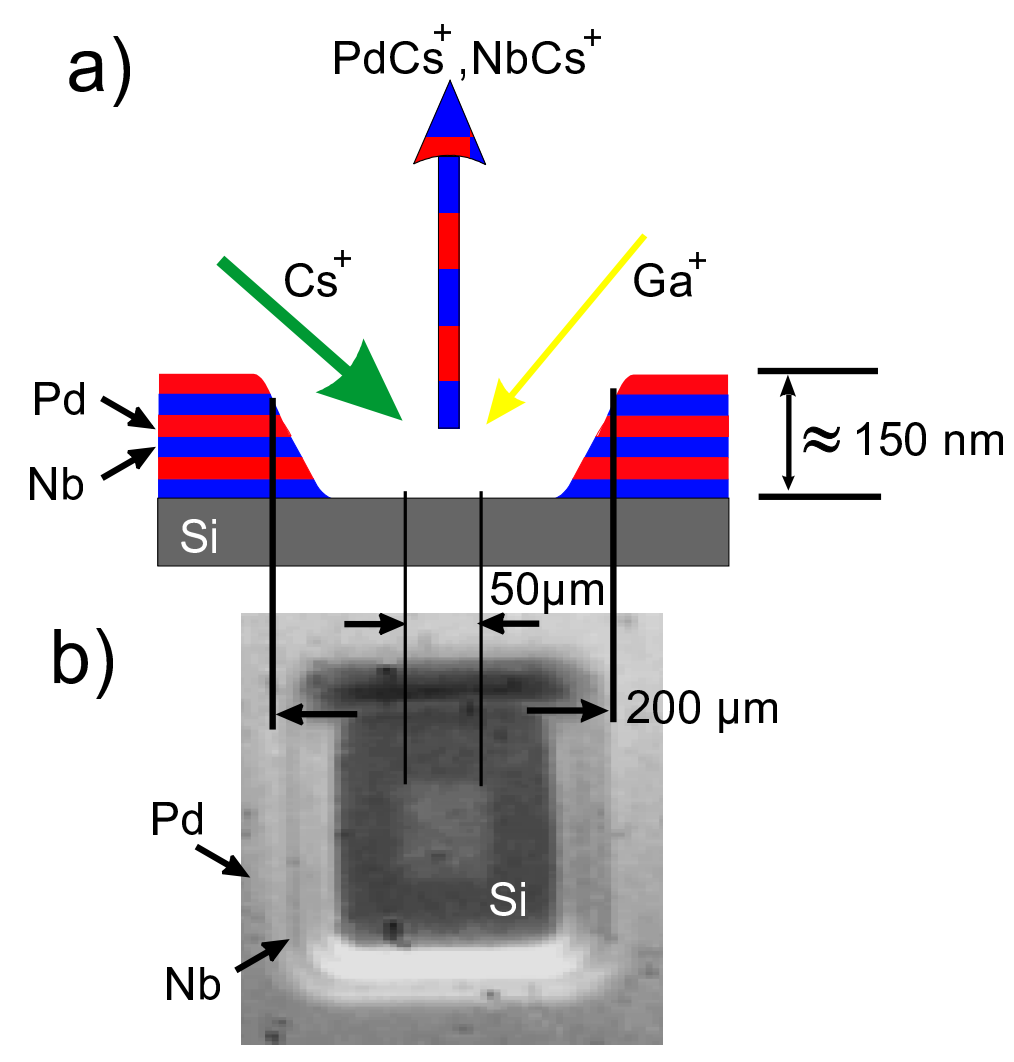

Abbildung 3.4: a) Prinzip der Tiefenprofilierung im SIMS. b) Foto eines Kraters nach dem Abtrag eines Pd/Nb-Schichtpaketes mit 3 Doppelschichten auf Si. Die Einzelschichtdicken betragen 25 nm, der Sputterkrater mißt (200 x 200), der Analysekrater (50 x 50) $\mu^{2}$. 
a)

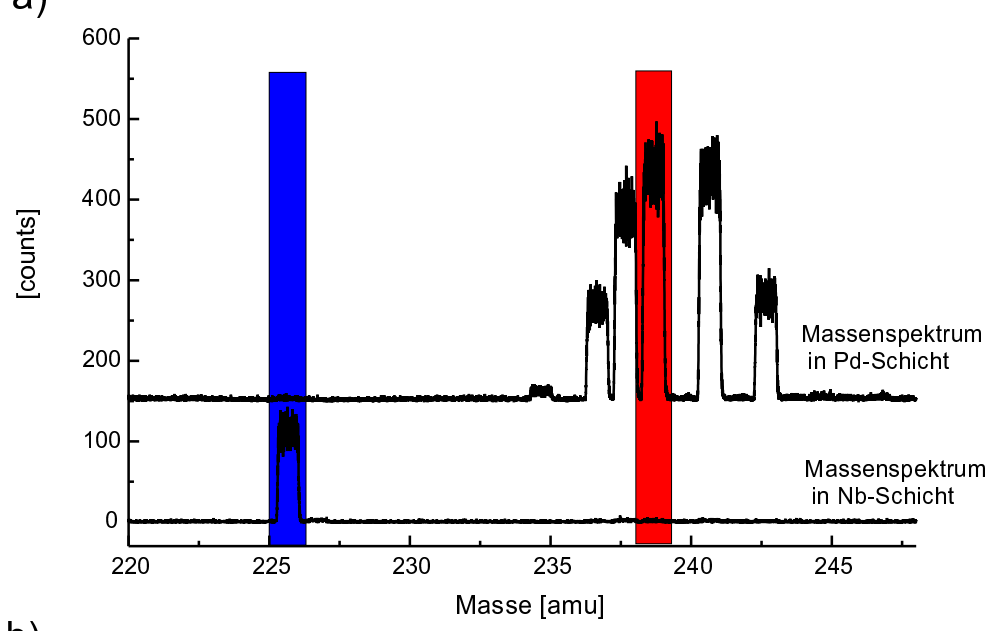

b)

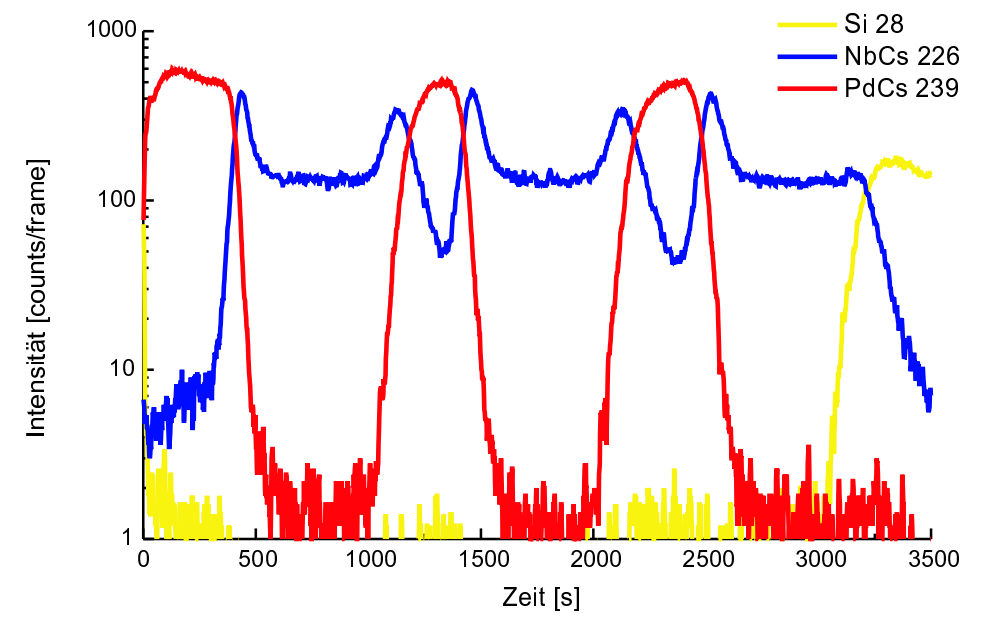

Abbildung 3.5: a) Ausschnitt der Massenspektren einer SIMS-Analyse. Die Peaks können dem NbCs und PdCs zugeordnet werden. Die obere Kurve ist um 150 counts nach oben versetzt und zeigt die detektierten Massen innerhalb einer Pd-Schicht. Die untere Kurve zeigt den gleichen Massenausschnitt in der nachfolgenden Nb-Schicht. Rot und blau eingezeichnet sind die ausgewählten Massenfenster, mit denen das Tiefenprofil b) aufgezeichnet wurde. Das untersuchte Schichtsystem ist das der Abb. 3.4b).

Abbildung 3.4 a) veranschaulicht das Zusammenspiel von Cs-Sputter- und Ga-Analyse-Ionenstrahl bei der Tiefenprofilierung im SIMS. Während der Sputterstrahl den Krater gräbt, wird mit dem Ga-Strahl auf dem Kraterboden analysiert. Die Tiefe des Kraters ist stark vergrößert dargestellt, sie liegt typischerweise im Bereich von $100 \mathrm{~nm}$, während die Kraterbreiten im Bereich von $200 \mu \mathrm{m}$ liegen. Abbildung 3.4 b) zeigt ein Foto eines Kraters in einem Pd/Nb-Schichtpaket mit 3 Doppelschichten. Der größere Sputterkrater mißt (200 x 200) $\mu m^{2}$, der kleinere 
Analysekrater (50 x 50) $\mathrm{mm}^{2}$, er befindet sich auf dem Boden des Sputterkraters. Am Kraterrand erkennt man die bereits abgetragenen Pd- und Nb-Schichten mit Einzelschichtdicken von $25 \mathrm{~nm}$. Im Krater ist das Si-Substrat zu erkennen.

Als Ergebnis der SIMS-Messungen erhält man zunächst die Anzahl der detektierten Ionen aus dem vom Ga-Strahl abgerasterten Bereich pro Massenfenster und Zeitinterval und damit der Probentiefe. Die Abbildung 3.5 a) zeigt jeweils den gleichen Massenausschnitt eines Histogramms, das von der SIMS-Messung am Pd/Nb-Multischichtsystem aus Abb. 3.4 b) stammt. Dabei unterscheiden sich nur die Zeitpunkte, d.h. die Probentiefe, in der die Massen detektiert wurden. Die aus Gründen der Übersichtlichkeit um 150 counts nach oben versetzte Kurve zeigt die Ionen innerhalb einer Pd-Schicht, die untere Kurve die detektierten Massen in der nachfolgenden Nb-Schicht. Durch geeignete Wahl der Massenfenster (blau für die Nb-Massen und rot für ein Pd-Isotop) lassen sich die gewonnen Daten auf gewünschte Intervalle beschränken und während des Abtrags der Schichten ein sogenanntes Tiefenprofil der ausgewählten Massen aufzeichnen. Als Ergebnis des Tiefenprofils erhält man die detektierten Ionen im jeweiligen Massenfenster pro Zeitinterval in ihrem zeitlichen Verlauf. Sinnvolles Zeitinterval ist dabei die Zeit für das Rastern der eingestellten Analysefläche, dem "frame" (typischerweise 50 x $50 \mu \mathrm{m}$ ), was ca. $2 \mathrm{~s}$ entspricht. Damit erhält man die Intensität der detektierten Ionen als "counts/frame", d.h. als Mittelwert über einen Analysezyklus. Abbildung 3.5 b) zeigt das Tiefenprofil, das mit den in Abb. 3.5 a) markierten Massenfenstern für Pd und $\mathrm{Nb}$ beim Abtrag des $\mathrm{Pd} / \mathrm{Nb}$-Schichtsystems aus Abb. 3.4 b) gewonnen wurde. Das Si-Signal in Abb. 3.4 b) in den Pd-Schichten ist als Untergrundsignal zu deuten.

Ein Fortschreiten in der Zeit durch das Absputtern der Schicht bedeutet gleichzeitig eine Messung in die Tiefe der Probe. Bei konstanter Abtragsrate ist die Zeit proportional zur Tiefe. In Multischichtsystemen mit in der Regel unterschiedlichen Raten in den unterschiedlichen Materialien ist dies nicht der Fall. Zudem ist in Durchmischungsgebieten die Rate unbekannt. Deswegen sind die Ergebnisse stets gegen die Zeit aufgetragen. Messungen der Schichten mit dem Profilometer und EDX und die daraus resultierenden Dicken werden zusätzlich angegeben. 


\section{Kapitel 4}

\section{Herstellung und \\ Charakterisierung dünner, metallischer Schichtsysteme}

Im folgenden Kapitel wird zunächst die Herstellung der metallischen Multischichtsysteme beschrieben. Es wurden Schichten aus den Metallkombinationen Pd/Nb, $\mathrm{Nb} / \mathrm{W}$ und $\mathrm{Fe} / \mathrm{V}$ hergestellt, die sich durch einen großem Unterschied in der H-Löslichkeit auszeichnen (s. Tab. 2.4). In diesen Proben sollten auftretende elektronische Grenzflächeneffekte deutlich zu Tage treten. Anschließend folgt die Charakterisierung der Schichtsysteme. Dabei liegt der Schwerpunkt in der Untersuchung der Grenzflächenrauhigkeit der hergestellten Schichtsysteme. Für die spätere Beurteilung der H-Verteilung an den Grenzflächen ist es notwendig, eventuell auftretende Durchmischungsbereiche zu charakterisieren, um elektronische Effekte an der Grenzfläche von chemischen trennen zu können.

\subsection{Herstellungsmethoden}

Zur Herstellung dünner Schichten mit Schichtdicken bis hinunter in den Subnanometerbereich haben sich Materialdepositionsverfahren etabliert, bei denen das zu deponierende Material als Atom- bzw. Ionenstrahl auf ein Substrat auftrifft und dort je nach Energie und Rate des Materialantransports und je nach Temperatur und Beschaffenheit des Substrates zum Aufwachsen von Schichten führt. Metallische Schichten wurden im Rahmen dieser Arbeit sowohl mit Hilfe der gepulsten Laserdeposition, als auch mittels Ionenstrahl-Sputterdeposition auf die Substrate aufgebracht. 


\subsubsection{Gepulste Laserdeposition (LD)}

Bei der LD handelt es sich um eine Methode, die sich vor allem durch die hohen kinetischen Energien (ca. $100 \mathrm{eV}$ ) der deponierten Materialien auszeichnet [52]. Diese Energien führen auf dem Substrat zu Sputtereffekten, die je nach Material zu erhöhten Defektdichten führen können [53, 54]. Dadurch erwartet man bei der Herstellung von Multischichten eine erhöhte Durchmischung an den Grenzflächen im Vergleich zu energieärmeren Methoden. Dies erhöht die Haftung der Schichten auf dem Substrat, die für die Feldionenmikroskopie von entscheidener Bedeutung ist.

Die laserdeponierten Proben wurden von M. Weisheit (Institut f. Materialphysik) hergestellt. Die Herstellung erfolgte im UHV bei Raumtemperatur. Das Vakuum in der Depositionskammer betrug ca. $10^{-8}$ mbar und stieg während der Deposition auf ca. $10^{-6}$ mbar. Der Druckanstieg ist auf einen erhöhten HPartialdruck zurückzuführen, der durch die vom Laserbeschuß erwärmten Targets erzeugt wird. Pd und $\mathrm{Nb}$ wurden mit Laserenergien von 120-160 mJ und Raten von 1 bis $20 \mathrm{pm} /$ Puls aufgetragen. Hergestellte Multischichtpakete besaßen Gesamtdicken bis $200 \mathrm{~nm}$ und Einzelschichtdicken von 2 bis $30 \mathrm{~nm}$.

\subsubsection{Ionenstrahlsputterdeposition (SD)}

Die SD zeichnet sich durch geringere Depositionsenergien unterhalb von $10 \mathrm{keV}$ [55] und niedrigen momentanen Depositionsraten aus, was bei besonders guten Restgasvakua $\left(<10^{-11}\right.$ mbar) und hohen Depositionstemperaturen $\left(700^{\circ} \mathrm{C}\right) \mathrm{zu}$ epitaktischem Wachstum führen kann. Bei tieferen Temperaturen können auch Metallkombinationen aus mischenden Systemen mit geringer Durchmischung an den Grenzflächen aufgebracht werden. Durch die geringe Depositionsenergie wird ein Ionenmischen unterdrückt und die tiefen Temperaturen verhindern eine Interdiffusion und -reaktion benachbarter Elemente.

Die gesputterten Schichten wurden in zwei verschiedenen Anlagen hergestellt. Anlage A mit einem Restgasvakuum von $10^{-10}$ mbar bietet die Möglichkeit die Substrate bis zu Temperaturen von $900^{\circ} \mathrm{C}$ zu heizen. In Anlage B, die speziell für die Beschichtung von FIM-Spitzen optimiert ist, kann nicht geheizt werden, dafür können mit dem Ar-Ionenstrahl auch die Substratspitzen gereinigt werden. Der Basisdruck in Anlage B war mit $10^{-8}$ mbar deutlich höher als in A. Die Autragsraten lagen für Anlage A zwischen 1-3 nm/min und in Anlage B bei 5-15 $\mathrm{nm} / \mathrm{min}$.

In den Sputteranlagen wurden sowohl Pd/Nb- als auch Fe/V-Schichtsysteme hergestellt. Die typischen Schichtdicken entsprachen denen der Laserdeposition. 


\subsection{Präparation der Schichtsysteme}

Die Analyse der hergestellten Schichtsysteme mit den in Kapitel 3 vorgestellten Techniken verlangt eine gezielte Präparation der Proben. Dies betrifft vor allem die Auswahl und Vorbehandlung des Substrates, die speziell für die analytische Feldionenmikroskopie sehr aufwendig ist, da Spitzen mit Krümmungsradien kleiner als $100 \mathrm{~nm}$ benötigt werden.

Zur Bestimmung der mittleren Einzelschichtdicken wurde bei allen Herstellungsverfahren als Referenzsystem ein Si-Substrat mitbeschichtet. Die Gesamtschichtdicke auf dem Si-Substrat wurde anschliessend mit einem Profilometer ausgemessen und die Konzentration der aufgebrachten Metalle mittels energiedispersiver Röntgenfluoreszensanalyse (EDX) bestimmt. Aus diesen Daten kann man bei Kenntnis bzw. Näherung des Molvolumens für die verwendeten Metalle, die mittlere Einzelschichtdicke mit einem Gesamtfehler von ca. $10 \%$ abschätzen. Bei den Schichten auf den gekrümmten Spitzen ergab sich durch Vergleichsmessungen mit der Transmissionselektronenmikroskopie (TEM) eine um ca. die Hälfte reduzierte Gesamtschichtdicke im Vergleich zum Si-Referenzsystem. Die Schichtdicken der FIM/TAP-Messungen werden deshalb alle unter Berücksichtigung dieses Faktors angegeben. Der Fehler der Schichtdickenbestimmung erhöht sich in diesen Systemen auf ca. $50 \%$.

\subsubsection{Präparation für SIMS}

Mit vergleichsweise geringem präparativen Aufwand lassen sich die Proben für die SIMS-Analysen herstellen. Es wurden $\mathrm{Pd} / \mathrm{Nb}$-Schichtsysteme auf planare, polierte Si-Scheiben der Stärke 0,5 mm mit [100]-Textur aufgebracht. Die Größe der Substrate reichte von $7 \times 7 \mathrm{~mm}^{2}$ bis zu Flächen von $3 \mathrm{~cm}^{2}$. Alle Systeme wurden mit einer 5 bis $20 \mathrm{~nm}$ dicken Pd-Deckschicht abgeschlossen, um die Proben vor Oxidation zu schützen und die H-Beladung zu ermöglichen. An einem Teil der Proben wurde als erste Schicht auf dem Si-Substrat eine Pd-Schicht als Puffer aufgebracht.

\section{Struktur der hergestellten Systeme}

Zur Bestimmung der Wachstumsrichtungen der $\mathrm{Pd} / \mathrm{Nb}-\mathrm{Schich}$ ten wurden von M. Dornheim [56] Röntgen-Texturmessungen an Pd/Nb-Doppelschichten durchgeführt. Abbildung 4.1 zeigt die [110]-Polfigur einer laserdeponierten und einer gesputterten Nb-Schicht von jeweils $200 \mathrm{~nm}$ Dicke mit einer Pd-Deckschicht von $100 \mathrm{~nm}$. Man erkennt im Zentrum der Polfigur jeweils die hohe Intensität durch eine starke [110]-Texturierung der Nb-Körner senkrecht zur Schicht. Die Ringstrukturen belegen die isotrope Verteilung der anderen $<110>$-Richtungen in der Schichtebene. Diese Ergebnisse bestätigen die von Laudahn [7] an laserdeponierten Schichten gefundene [110]-Vorzugsrichtung. 
laserdeponiert

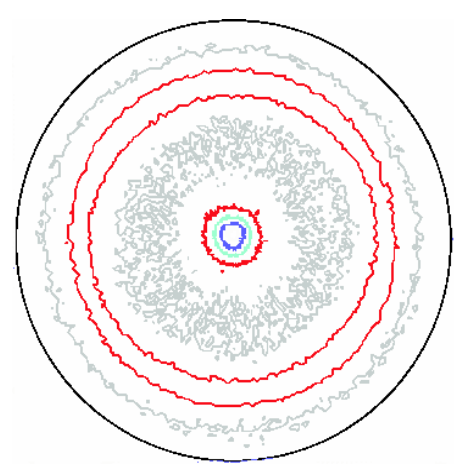

gesputtert

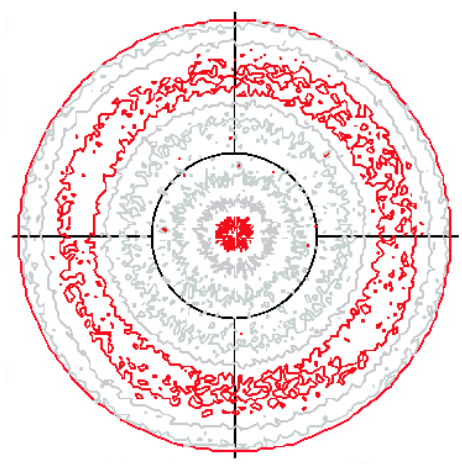

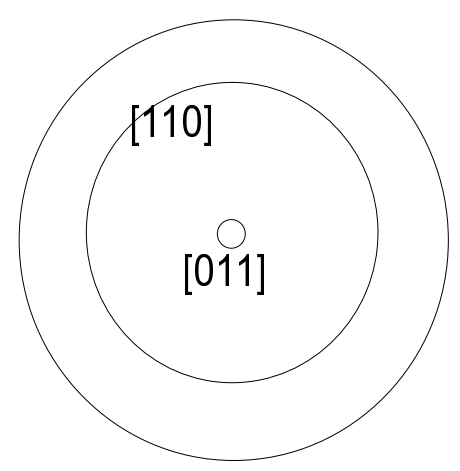

Abbildung 4.1: [110]-Polfigur einer laserdeponierten und einer gesputterten $\mathrm{Nb}$ Schicht von jeweils $200 \mathrm{~nm}$ Dicke und Pd-Deckschicht von $100 \mathrm{~nm}$ für die laserdeponierte und $25 \mathrm{~nm}$ für die gesputterte Schicht [56]. Man erkennt eine starke [110]-Texturierung der Nb-Schicht, sowohl bei der laserdeponierten Schicht, als auch bei der gesputterten. Die Ringstrukturen belegen die isotrope laterale Verteilung der [110]-Körner.

Die HREM-Aufnahme (Abb. 4.2) von Borchers et al. [57] an querschnittspräparierten, laserdeponierten $\mathrm{Pd} / \mathrm{Nb}$-Schichten zeigt die Gitterfringes der $\{110\}$ Ebe-nen im Nb und der $\{111\}$-Ebenen im Pd und bestätigt damit die Ergebnisse der Texturmessungen. Weitere Untersuchungen ergeben, daß die Korngröße sowohl im Nb, als auch im Pd im Bereich der Einzelschichtdicke liegt. Eingezeichnet sind zusätzlich die Versetzungen in Strahlrichtung, die mit Hilfe einer Fourieranalyse der Fringes und Rücktransformation ermittelt wurden.

\subsubsection{Präparation für FIM und TAP}

Ein nicht zu vernachlässigendes Problem stellt die Präparation der Schichtsysteme für die feldionenmikroskopischen Methoden dar. Zum einen muß das System die für die Feldionenmikroskopie benötigte komplexe nanoskopische Spitzenform besitzen und gleichzeitig die nötige Stabilität, um den extremen mechanischen Belastungen der angelegten Feldstärken standzuhalten. Die Spitzenform mit Spitzenradien von 20-50 nm erreicht man durch Aufbringen der Schichten auf entsprechend vorgeformte Substrate. Als Substrate für die FIM-Proben wurden WSpitzen benutzt (vgl. [58, 55]. W-Drahtstücke wurden elektrolytisch zu Spitzen mit Krümmungsradien von ca. 20-50 nm gedünnt. Als Elektrolyt wurde $\mathrm{NaOH}$ (2 N) verwendet und die Spitzen pulsweise mit Wechselspannungen zwischen 2 und $5 \mathrm{~V}$ bei Raumtemperatur gedünnt. Die W-Spitzen wurden anschliessend im FIM bei 30 - $100 \mathrm{~K}$ mit Spannungen von 4 - $9 \mathrm{kV}$ unter $5 \cdot 10^{-5}$ mbar He ent- 


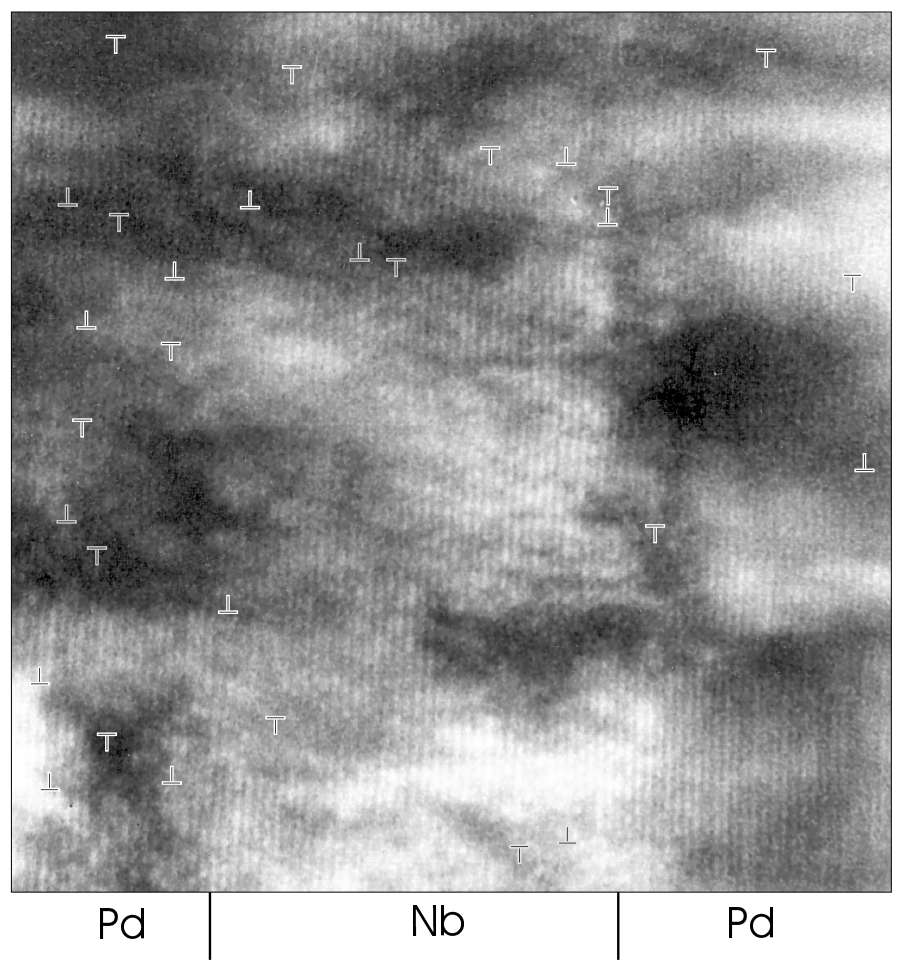

Abbildung 4.2: a) HREM-Aufnahme der $\{110\}$-Nb-und der $\{111\}$-Pd-Ebenen eines laserdeponierten Schichtsystems mit einer Doppelschichtdicke von $22 \mathrm{~nm}$ [57]. Eingezeichnet sind die Versetzungen in Strahlrichtung, die mit Hilfe einer Fourieranalyse der Fringes und Rücktransformation ermittelt wurden.

wickelt. Dabei wurde nicht nur die Stabilität der W-Spitze geprüft, sondern durch Feldverdampfung vor allem die Oberfläche des Substrates gereingt und auf für die Detektion benötigte Radien entwickelt, d.h. durch Feldverdampfung vorgeformt. Eine entsprechend präparierte W-Spitze zeigt Abb. 4.3. Die Spitze hat am Apex einen Radius von $20 \mathrm{~nm}$ und wurde im FIM bei $30 \mathrm{~K}$ bis $4,5 \mathrm{kV}$ entwickelt.

Die Stabilität der Schichtsysteme und somit ihre Tauglichkeit für FIM- bzw. Atomsonden-Untersuchungen hängt entscheidend von der Vorbehandlung der Substrate ab, wird aber auch durch die gewählte Depositionsmethode beeinflußt. Was die Vorbehandlung der Substrate betrifft, so zeigte sich, daß die nötige Stabilität überhaupt erst erreicht wird, wenn man die W-Substrate vor der Deposition im FIM entwickelt. Nach der Entwicklung wird beim Einbau in die Depositionskammer das Vakuum wieder gebrochen, was zur Oxidation und eventuell zu weiteren Verunreinigungen der Oberflächen führt. In Sputteranlage B kann vor der Deposition die Spitzenoberfläche im Ar-Ionenstrahl erneut gereinigt werden. Im Falle der Laserdeposition und Sputterdeposition in Anlage A war eine Reinigung nicht möglich. Dies wirkte sich in einer geringeren Ausbeute FIM-stabiler Schichtsysteme aus. 


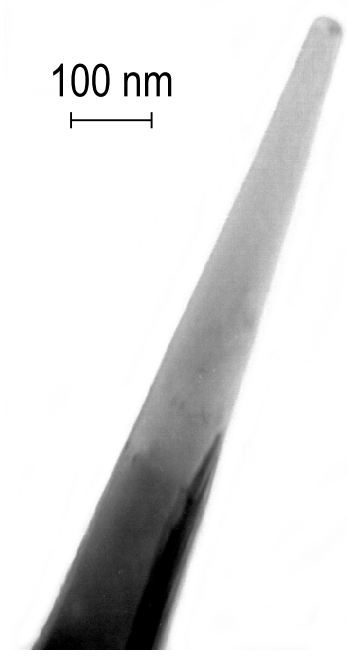

\begin{abstract}
Abbildung 4.3: TEM-Aufnahme einer im FIM bei $6 \mathrm{kV}$ und $30 \mathrm{~K}$ entwickelten $W$-Spitze mit einem Spitzenradius von $20 \mathrm{~nm}$.
\end{abstract}

Die entwickelten W-Substrate wurden anschließend in die Depositionskammer eingebaut. $\mathrm{Pd} / \mathrm{Nb}-\mathrm{Schich}$ tsysteme wurden sowohl mit Laserdeposition als auch mit Sputterdeposition hergestellt. Dabei wurde auf das W-Substrat als erstes eine Nb-Schicht aufgebracht und das System mit einer Pd-Schicht abgeschlossen. Die Fe/V-Systeme wurden ausschließlich gesputtert. Auf das W-Substrat wurde zunächst eine Fe-Schicht aufgebracht und die abschliessende V-Schicht noch mit einer Pd-Deckschicht vor Oxidation geschützt.

Bei der Laserdeposition wurden die W-Substrate während des Auftrages um die Spitzenachse rotiert, die sich im Winkel von $20^{\circ}$ zum Target befand. Dies wurde in früheren Arbeiten als gute Konfiguration ermittelt. Zur Kontrolle der Deposition wurde auf dem Spitzenhalter, der 6 Spitzen aufnehmen kann, auch ein planares Si-Substrat befestigt.

In der Sputteranlage A wurden die Proben mit Ar der Reinheit 99,9997\% bei ca. $1 \cdot 10^{-4}$ mbar und bei Temperaturen von $320^{\circ} \mathrm{C}$ hergestellt. Auf dem Probenhalter, der 4 Spitzen aufnehmen kann, wurde ebenfalls ein Si-Substrat befestigt. Die Proben wurden bei $320^{\circ} \mathrm{C}$ ausgeheizt und während der Deposition um die Spitzenachse rotiert. Die Spitzenachsen waren senkrecht zum drehbaren Targethalter ausgerichtet.

In Anlage B wurden die Spitzen zunächst im Ar-Strahl gereinigt und anschliessend mit Ar der Reinheit 99,9997\% bei ca. $3 \cdot 10^{-4}$ mbar bei RT hergestellt. Auch hier diente ein Si-Substrat als Kontrollsystem.

Eine Abschätzung der Gesamtdicke der Schichtsysteme erhält man zusätzlich durch TEM nach der Deposition. Dies ist insofern wichtig, da die aufgebrachten Schichten auf der gekrümmten W-Oberfläche durch Sputtereffekte dünner sind. Sie zeigten sich im Mittel nur halb so dick wie die des beschichteten Si-Substrates. 


\section{Struktur der hergestellten Systeme}

Die Spitzen wurden nach der Deposition im TEM untersucht. Abb. 4.4 zeigt ein laserdeponiertes Pd/Nb-Doppelschichtsystem mit einer Gesamtdicke von $40 \mathrm{~nm}$. Mit dem Pfeil markiert ist der dunklere Bereich des W-Substrates, das im Zustand vor der Beschichtung in Abb. 4.3 dargestellt ist. Die Schichten wachsen auf der Spitze am stärksten und zeigen dort auch eine gleichmäßige Oberfläche. Den Schaft entlang wird das Wachstum rauher und geringer. Abb. 4.5 zeigt ein in Anlage B gesputtertes Fe/V-Doppelschichtsystem mit Pd-Deckschicht und einer Gesamtdicke von $40 \mathrm{~nm}$. Die im TEM erkennbare Struktur unterscheidet sich nur unwesentlich von der der laserdeponierten Spitze. Der Spitzenradius beträgt 35 nm. Mit dem FIM lassen sich zusätzlich zu den in Abschnitt 4.2.1 beschriebenen
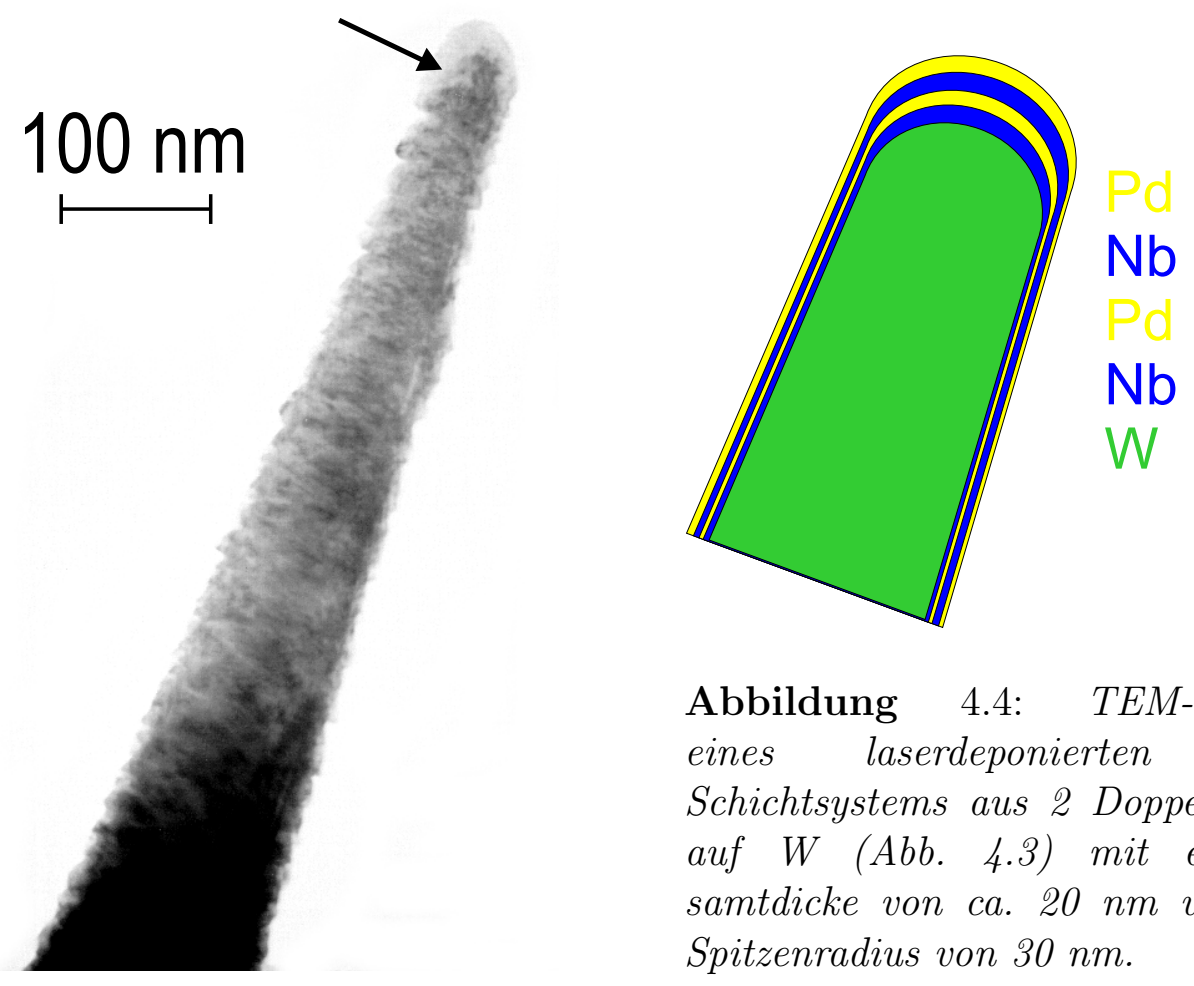

Abbildung 4.4: TEM-Aufnahme
eines laserdeponierten Pd/Nb-
Schichtsystems aus 2 Doppelschichten
auf W (Abb. 4.3) mit einer Ge-
samtdicke von ca. $20 \mathrm{~nm}$ und einem
Spitzenradius von $30 \mathrm{~nm}$.

strukturellen Eigenschaften der auf Si deponierten Pd/Nb-Multischichtsysteme auch Aussagen über die Struktur auf Nanometerskala machen. Da als Substrat hier eine W-Oberfläche benutzt wird, sind die Informationen nicht direkt übertragbar. FIM-Aufnahmen (Abb. 4.6) der letzten Nb-Schicht (a)) auf dem WSubstrat (b)) des Pd/Nb-Schichtpakets der Abb. 4.4 veranschaulichen jedoch in Übereinstimmung mit den Ergebnissen der planaren Systeme, daß Nb in [110]Richtung auf W aufwächst. Das W-Substrat selbst ist in der Spitzenachse ebenfalls [110]-orientiert. Die Ringstruktur des W-Bildes zeigt dementsprechend eine zweizählige Symmetrie. An Hand der ungestörten Ringstruktur erkennt man, daß 

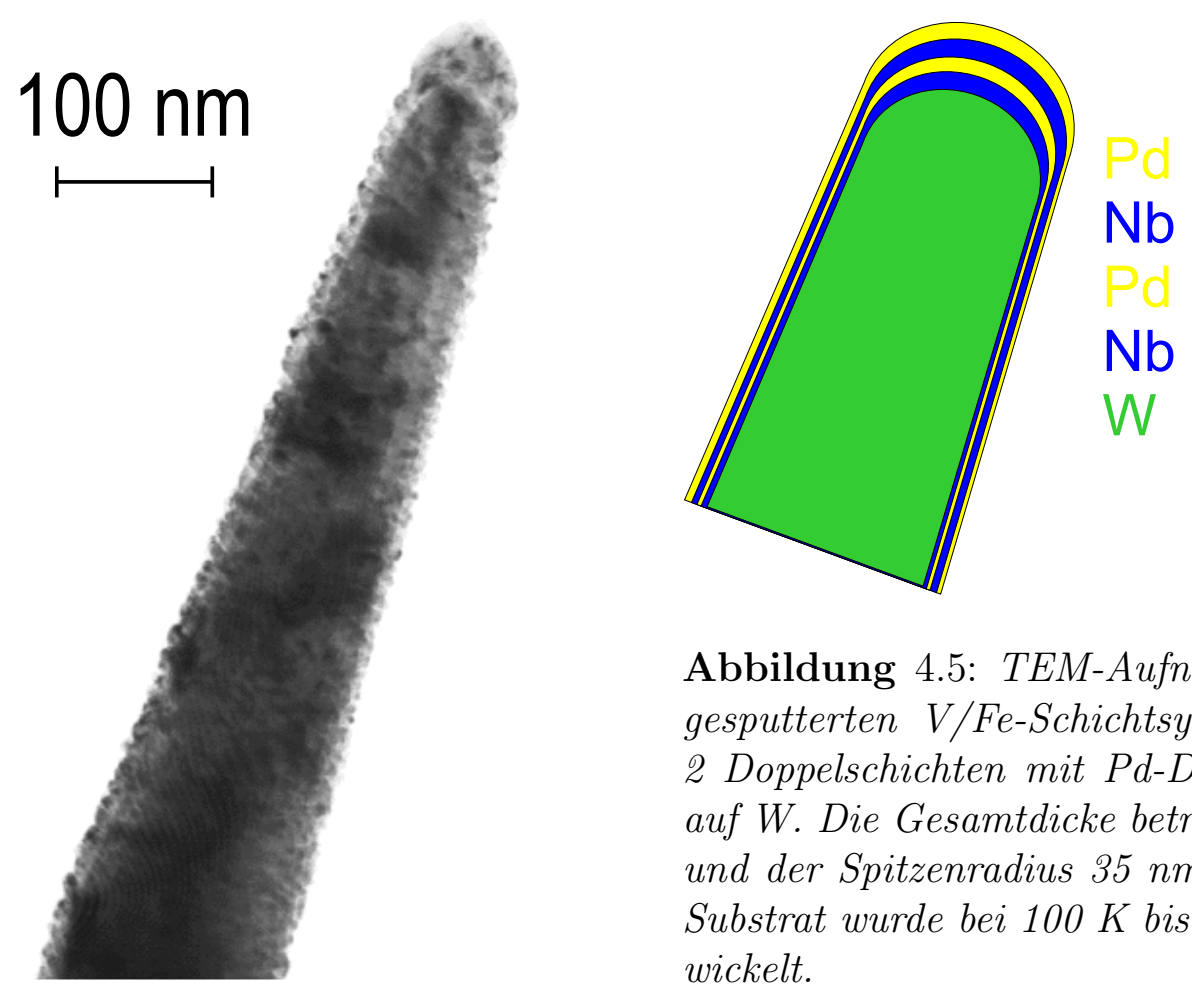

Abbildung 4.5: TEM-Aufname eines
gesputterten V/Fe-Schichtsystems aus
2 Doppelschichten mit Pd-Deckschicht
auf W. Die Gesamtdicke beträgt $40 \mathrm{~nm}$
und der Spitzenradius $35 \mathrm{~nm}$. Das W-
Substrat wurde bei $100 \mathrm{~K}$ bis $6 \mathrm{kV}$ ent-
wickelt.

es sich im W um einen einkristallinen Bereich handelt (4.6b)). Die Indizierung der W-Pole ist in Teil c) abgebildet. Die Polstruktur des Nb ist nicht so scharf wie die des Substrates, man erkennt jedoch deutlich ein Aufeinanderfallen des zentralen und der beiden linken [110]-Pole der Nb-Schicht auf die des Substrats. Daraus läßt sich schließen, daß der im FIM untersuchte Bereich der Schichten von einigen $100 \mathrm{~nm}^{2}$ epitaktisch aufwächst. Für die Fe/V-Systeme kann diese Aussage an Hand von FIM-Bildern nicht belegt werden, da die untersuchten Systeme zu dünn für eine deutliche FIM-Abbildung waren. 
a)

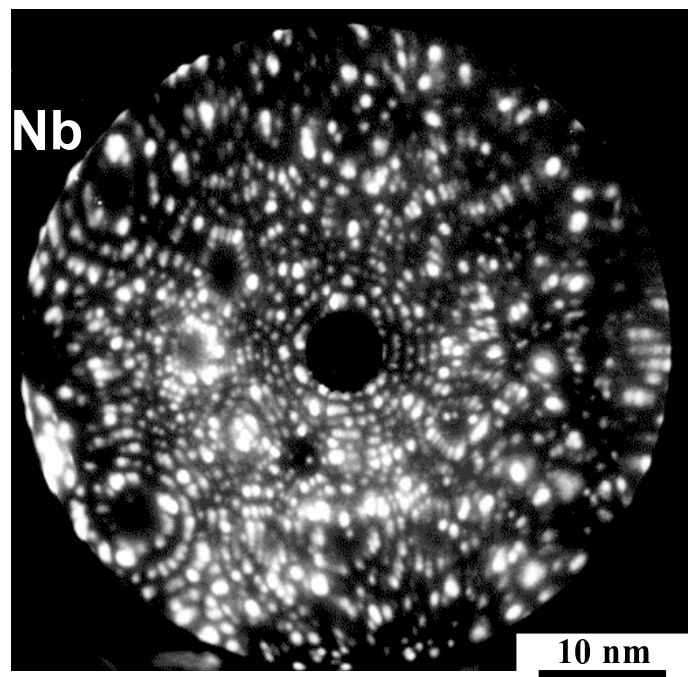

b)
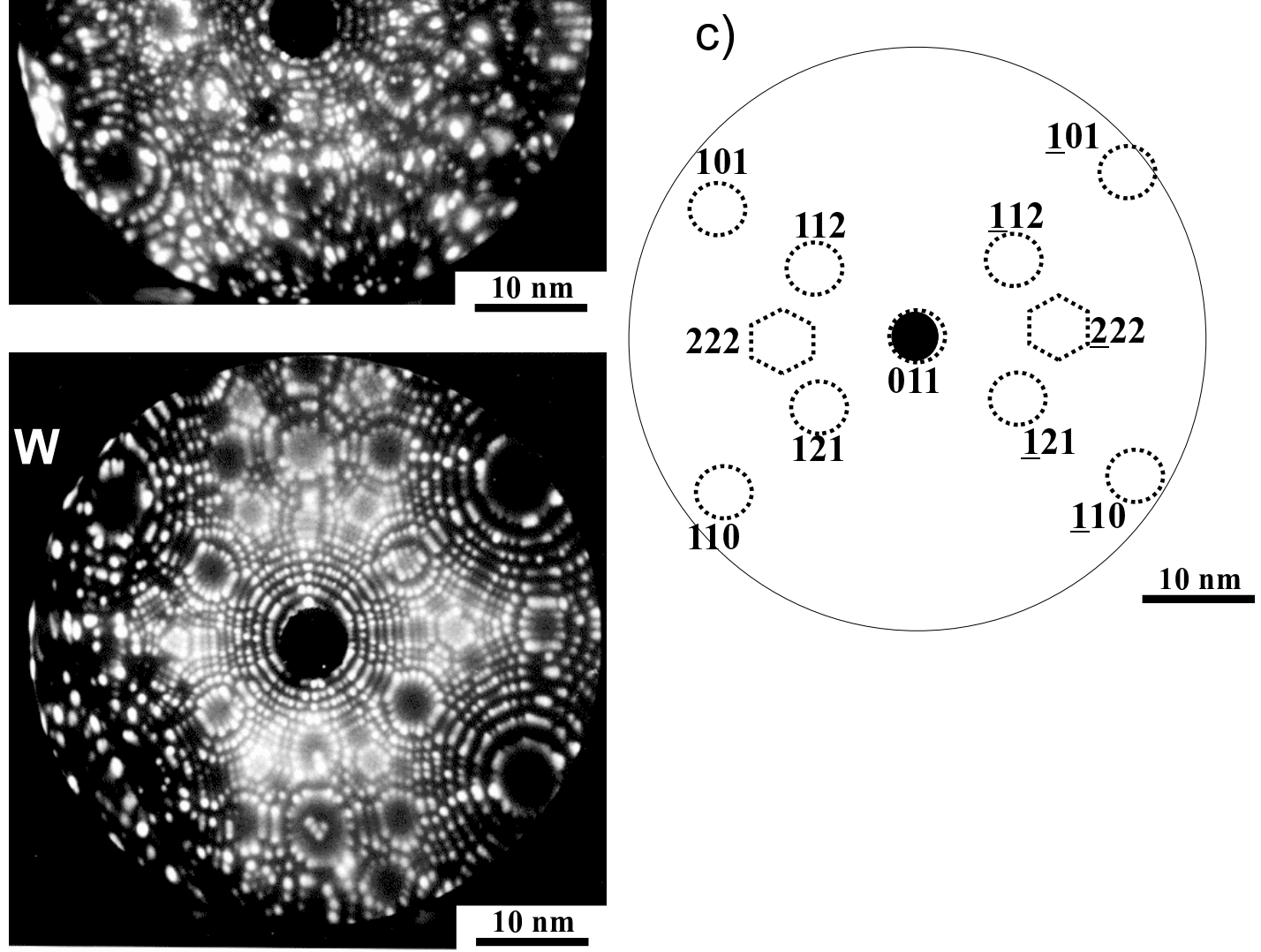

Abbildung 4.6: FIM-Bild einer Nb-Schicht (a) bei 3,6 kV und des darunterliegenden $W$-Substrates bei $4,0 \mathrm{kV}$, abgebildet bei $70 \mathrm{~K}$ mit He und Ne als Bildgas (Gesamtdruck $5 \cdot 10^{-5}$ mbar, He:Ne-Verhältnis 1:1,5). Die FIM-Bilder stammen von der Probe aus Abb. 4.4. 


\subsection{Einfluß der Herstellungsmethode auf die Grenzflächenschärfe der Multischichtsysteme}

Im folgenden Abschnitt sollen die Untersuchungen der Pd/Nb- und Fe/V-Schichtsysteme mit dem SIMS und TAP dargestellt werden, die an unbeladenen Proben durchgeführt wurden. Die Ergebnisse sollten zum einen Informationen über die Auflösung der eingesetzten Methoden an den metallischen Grenzflächen ohne den Einfluß von Wasserstoff liefern. Und zum anderen sollte die Abhängigkeit der chemischen Grenzflächenschärfe von den eingesetzten Depositionsverfahren geklärt werden. Da sich die gepulste Laserdeposition und die Sputterdeposition in der Energie der auftreffenden Teilchen um eine Größenordnung unterscheiden, ist eine breitere Durchmischung an der Grenzfläche der laserdeponierten Schichten als an der gesputterter Schichten zu erwarten.

\subsubsection{Das System Pd/Nb}

Mit dem SIMS wurden sowohl laserdeponierte als auch gesputterte $\mathrm{Pd} / \mathrm{Nb}$-Schichtsysteme analysiert. Die Methode ist in Abschnitt 3.2 beschrieben. Abb. 4.7 zeigt das Tiefenprofil eines laserdeponierten Multischichtsystems aus $6 \mathrm{Pd} / \mathrm{Nb}-$ Doppellagen, Abb. 4.8 das eines gesputterten Systems aus 5 Pd/Nb-Doppellagen mit Pd-Pufferschicht auf Si.

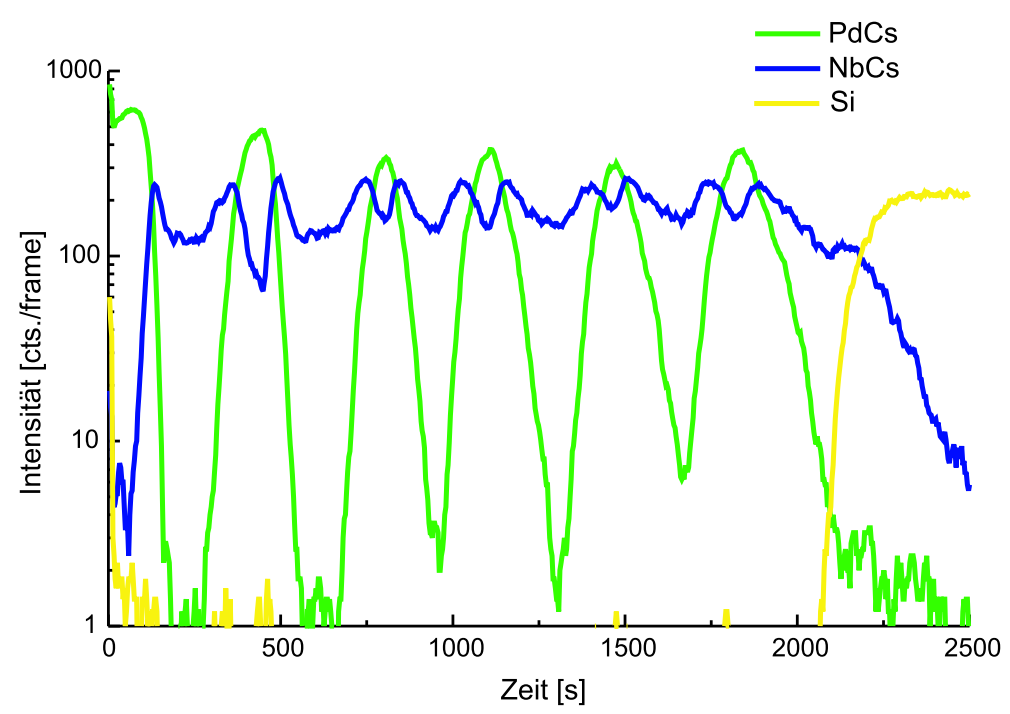

Abbildung 4.7: SIMS-Tiefenprofil eines laserdeponierten $\mathrm{Pd} / \mathrm{Nb}$ Multischichtsystems aufgenommen auf einer Fläche von $\left(\begin{array}{llll}50 & x & 50\end{array}\right) \mathrm{m}^{2}$ mit Ga-Ionen. Zum Tiefenabtrag wurde mit Cs-Ionen ein Krater von (200 x 200) $\mu m^{2}$ gesputtert. Das System besteht aus 6 Doppellagen mit Einzelschichtdicken von jeweils 12,5 nm. Aufgetragen ist die Zählrate von PdCs, NbCs und Si gegen die Meßzeit und somit Tiefe der Probe. 


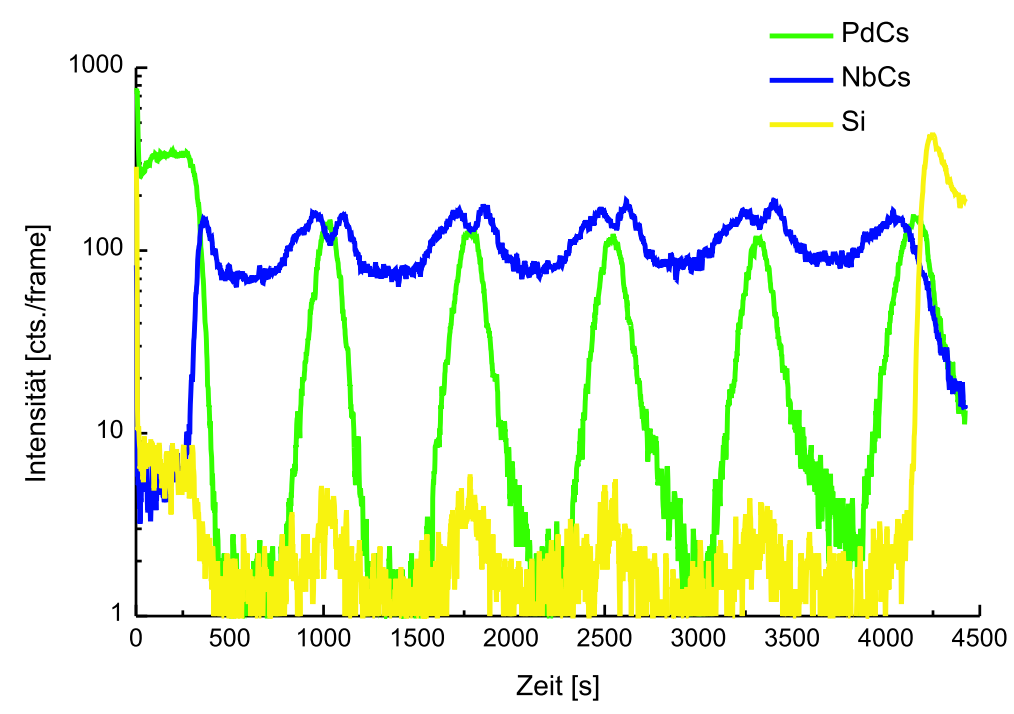

Abbildung 4.8: SIMS-Tiefenprofil eines gesputterten $\mathrm{Pd} / \mathrm{Nb}$ Multischichtsystems aufgenommen auf einer Fläche von $\left(\begin{array}{lll}50 & x & 50\end{array}\right) \quad \mu^{2}$ mit Ga-Ionen. Zum Tiefenabtrag wurde mit Cs-Ionen ein Krater von (200 $x$ 200) $\mu m^{2}$ gesputtert. Das System besteht aus 5 Doppellagen und einer PdPufferschicht mit Pd-Schichtdicken von 8,3 $\mathrm{nm}$ und Nb-Dicken von 14,5 $\mathrm{nm}$. Aufgetragen ist die Zählrate von PdCs, NbCs und Si gegen die Meßzeit und somit Tiefe der Probe.

Aufgetragen sind die Zählraten der detektierten PdCs-, NbCs- und Si-Ionen, die nach $[49,50]$ mit den Konzentrationen korreliert sein sollten. Die Pd-Schichten erscheinen auf der Zeit-Achse dünner. Eine Analyse mit EDX und Profilometer ergab jedoch im Rahmen des Fehlers von maximal $10 \%$ gleich dicke Pd- und NbSchichten von 12,5 nm für das laserdeponierte Schichtpaket (Abb. 4.7). Daraus folgt, daß die Abtragsrate des SIMS in den Pd-Schichten höher als die in den NbSchichten ist. Das gesputterte Paket besteht nach EDX und Profilometeranalyse aus Einzelschichten von 14,5 nm Nb und 8,3 nm Pd.

Außerdem erkennt man in beiden Profilen eine Erhöhung der NbCs-Zählrate an den Grenzflächen zu den Pd-Schichten. Dies ist auf einen sogenannten Matrixeffekt, d.h. auf die bevorzugte Ionisierung in diesem Fall des NbCs in der Pd-Umgebung zurückzuführen. Dieser Effekt läßt sich in den Profilen korrigieren, indem man die PdCs- bzw. NbCs-Intensitäten als Bruchteil der Gesamtintensität aus PdCs, NbCs und Si aufträgt. Abbildung 4.9 zeigt die erste Doppelschicht der Messung des laserdeponierten Systems (Abb. 4.7) in einem solchen Konzentrationsprofil, Abbildung 4.10 die erste Doppelschicht des gesputterten Systems (Abb. 4.8).

Betrachtet man die Profile der SIMS-Messungen, so fällt auf, daß die Signale an den Grenzflächen nicht senkrecht abfallen. Außerdem findet man in der PdSchicht der gesputterten Probe, die mit 8,3 nm dünner als bei der laserdeponierten 


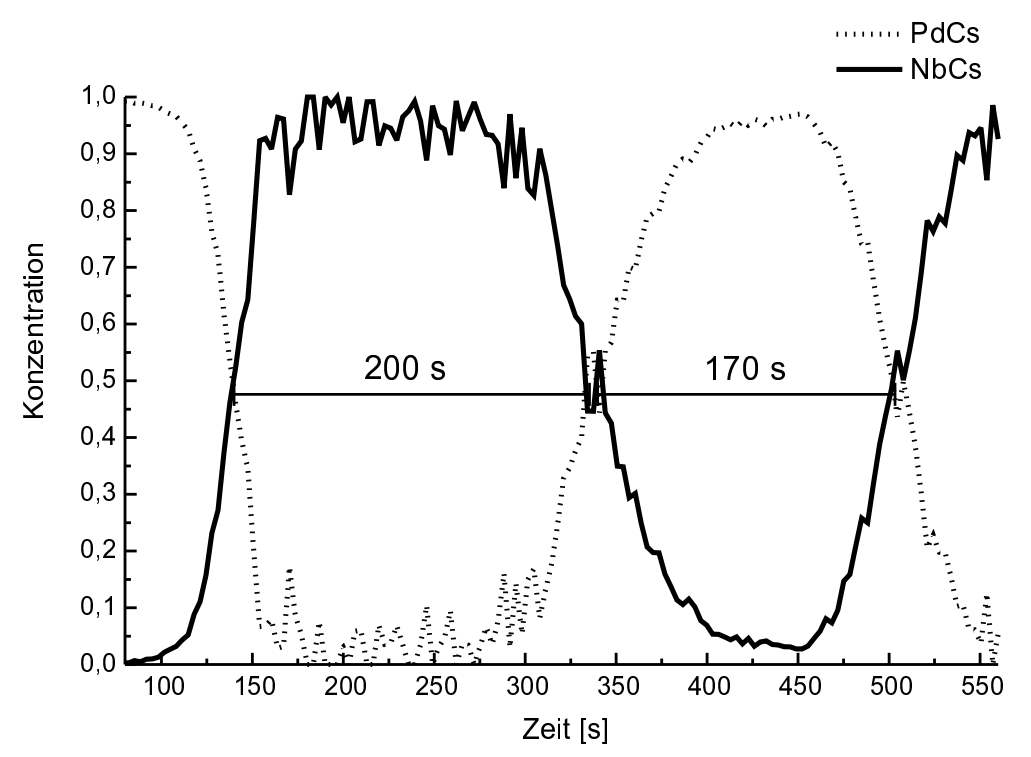

Abbildung 4.9: Konzentrationsprofil einer laserdeponierten $\mathrm{Pd} / \mathrm{Nb}$ Doppelschicht, berechnet aus den Zählraten der Messung aus Abb. 4.\%. Aufgetragen ist die Konzentration der gemessenen PdCs und NbCs-Signale für die erste Doppelschicht. Die Nb- und Pd-Schicht besitzt jeweils eine Dicke von 12,5 nm. Eingezeichnet sind zusätzlich die Halbwertsbreiten.

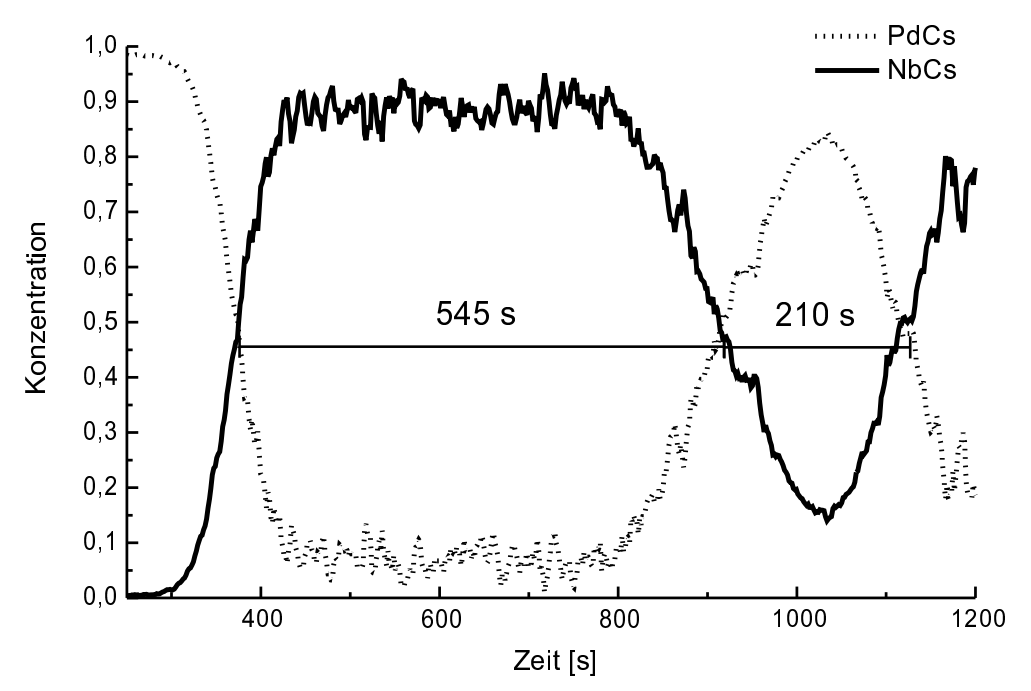

Abbildung 4.10: Konzentrationsprofil einer gesputterten Pd/Nb-Doppelschicht, berechnet aus den Zählraten der Messung aus Abb. 4.8. Aufgetragen ist die Konzentration der gemessenen PdCs und $\mathrm{NbCs-Signale} \mathrm{für} \mathrm{die} \mathrm{erste} \mathrm{Doppelschicht.}$ Die Nb-Schicht besitzt eine Dicke von 14,5 nm, Pd eine Dicke von 8,3 nm. Eingezeichnet sind zusätzlich die Halbwertsbreiten. 
Probe war, daß die Nb-Konzentration in der Pd-Schicht nicht auf Null abfällt, sondern bei ca. 15\% wegen der darauffolgenden Grenzfläche schon wieder ansteigt.

Zur Beurteilung des Abfalles der Signale an der Grenzfläche wird in der Literatur die Steigung der Flanken zwischen 84 und 16\% der Peakhöhe ausgewertet. Unter der Annahme, daß die gemessene Halbwertsbreite der Konzentrationskurven mit den mit EDX und Profilometer bestimmten Dicken übereinstimmt, lassen sich Aussagen über die Längenskala der Grenzflächenschärfe machen.

Die Abbildungen 4.9 und 4.10 wurden am Pd-Peak dementsprechend ausgewertet und die gefundenen Steigungen auf die Breite im Pd umgerechnet. Beim Übergang vom $\mathrm{Nb}$ ins $\mathrm{Pd}$ ergibt sich eine Übergangszone von $5 \mathrm{~nm}$ für das laserdeponierte System und für das gesputterte System von etwas unter $6 \mathrm{~nm}$. Beim Übergang vom $\mathrm{Pd}$ ins $\mathrm{Nb}$ findet man keinen Unterschied zwischen laserdeponiertem und gesputtertem System, beide Flanken verlaufen über ca. $4 \mathrm{~nm}$. Im Rahmen der Fehler in der Dickenbestimmung von ca. 10\% mit EDX und dem eher größeren Fehler durch den sehr einfachen Ansatz der Konzentrationsbestimmung bei der Auswertung der SIMS-Daten, ist auch die Abweichung von knapp $1 \mathrm{~nm}$ in der Grenzflächenunschärfe nicht signifikant. Insbesondere, da die Pd-Schicht im Fall der gesputterten Schicht (Abb. 4.10) dünner war und vor Erreichen eines Sputtergleichgewichts im Pd, das PdCs-Signal schon wieder durch den Einfluß der zweiten Grenzfläche absinkt.

Mit den SIMS-Messungen beobachtet man somit unter den gewählten Analysebedingungen, die für die Detektion der Metallcäsid-Komplexe optimiert waren, Grenzflächenunschärfen im Bereich von $5 \mathrm{~nm}$. Es läßt sich kein Unterschied in der Grenzflächenschärfe zwischen laser- und sputterdeponierten Nb/Pd-Schichten feststellen. Da es sich hier um eine Methode handelt, die durch Implantation der Primärionen zu Ionenmischen in der Probe führt, ist anzunehmen, daß die beobachtete Unschärfe ein Resultat der Analysemethode ist und nicht die wirkliche Grenzflächenschärfe wiedergibt. So ist je nach Ausmaß der Durchmischung durch die Analyse, ein Unterschied der Durchmischung der Grenzfläche, der durch die Deposition verursacht wird, nicht aufösbar.

Anzumerken ist, daß die abgebildeten Konzentrationsprofile sowohl der laserdeponierten als auch der gesputterten Schichten an der Nb/Pd- und an der $\mathrm{Pd} / \mathrm{Nb}$ den Ansatz einer Stufe bei Konzentrationen von ca. 50 at.\% zeigen. Dies ist ein Hinweis auf die Bildung einer NbPd-Phase. Dies wird im Rahmen der nun folgenden Darstellung der Ergebnisse der TAP-Messungen näher diskutiert.

Der Effekt des Ionenmischens durch die Analyse tritt bei einer TAP-Analyse nicht auf. Dadurch wird eine höhere Tiefenauflösung als im SIMS erzielt. Bei den Konzentrationsprofilen der TAP-Messungen müssen generell folgende Fehler berücksichtigt werden. Die Rekonstruktion der Daten ist durch viele Parameter beeinflußt, die man nicht exakt angeben kann, wie z.B. die Radien und Schaftwinkel der Spitze und die lokale Dichte der Probe. Der daraus resultierende Fehler kann für die absoluten Längenangaben bis zu $50 \%$ betragen. Bei den relativen Längen in unterschiedlichen Metallen ist der Fehler kleiner. Eine ausführliche Behandlung 
dieser Fehler folgt im Rahmen der Diskussion der Ergebnisse in Kapitel 5.

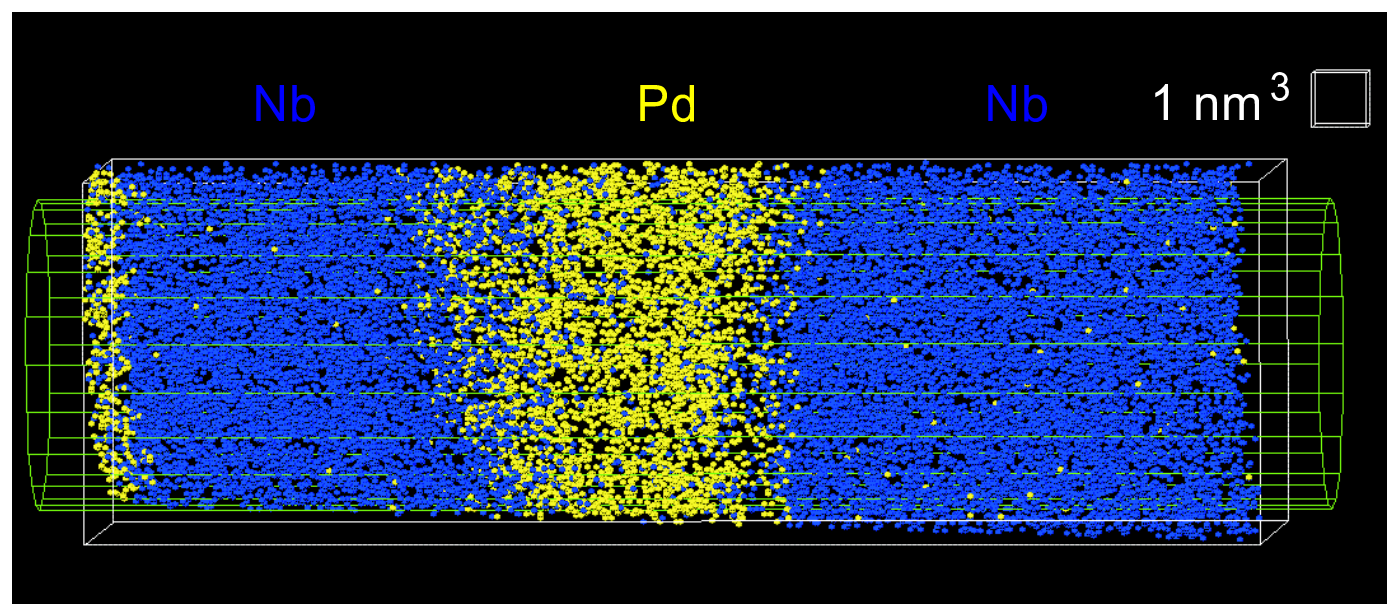

Abbildung 4.11: Rekonstruktion der TAP-Mesung von 2 Doppelschichten $\mathrm{Pd} / \mathrm{Nb}$. Hergestellt mit LD bei RT, gemessen bei $60 \mathrm{~K}$. Dargestellt sind die folgende Atomsorten: $\mathrm{Pd}$ (gelb) und $\mathrm{Nb}$ (blau). Das Volumen wurde mit einem Atomvolumen von $18^{3}$ rekonstruiert und beträgt $7 \times 7 \times 43 \mathrm{~nm}^{3}$. Der eingezeichnete Zylinder markiert das Teilvolumen, in dem die Konzentrationsanalyse durchgeführt wurde (Abb. 4.12). Er hat einen Radius von $3 \mathrm{~nm}$. Die mittlere Pd-Schicht zeigt eine deutliche Asymmetrie: links ist die Grenzfläche stärker interdiffundiert als rechts.

Abbildung 4.11 zeigt die rekonstruierte Messung einer laserdeponierten Probe aus zwei Pd/Nb-Doppelschichten. Die detektierten Pd-Atome sind gelb, die NbAtome blau dargestellt. Die erste Pd-Schicht wurde nicht vollständig gemessen. Das Volumen wurde mit einem Atomvolumen von $18{ }^{3}$ rekonstruiert und beträgt $7 \times 7 \times 43 \mathrm{~nm}^{3}$. Im ebenfalls eingezeichneten Zylinder mit einem Radius von $3 \mathrm{~nm}$ wurde eine Konzentrationsanalyse durchgeführt. Dazu wurde in einem Teilvolumen die Konzentration der enthaltenen Atome bestimmt. Das Analysevolumen bestand jeweils aus einer Scheibe des Zylinders mit einer Tiefe von 0,24 nm. Die mittlere Anzahl der Atome im Analysevolumen war größer als 100. Das Analysevolumen wurde schrittweise um $0,08 \mathrm{~nm}$ verschoben, so daß die ermittelten Konzentrationen ein gleitendes Mittel über die Tiefe der Probe darstellen.

Man erkennt im Konzentrations-Tiefenprofil dieser Messung (Abb. 4.12), daß die zwischen den beiden Nb-Schichten (blau) liegende Pd-Schicht (gelb) einen asymmetrischen Konzentrationsverlauf zeigt. Der Pd-Anstieg verläuft hier über eine Breite von fast $4 \mathrm{~nm}$ und zeigt bei ca. $50 \% \mathrm{Pd}$ ein zusätzliches Abflachen, das auf eine dünne NbPd-Phase hindeutet. Der Abfall zur zweiten Nb-Schicht verläuft dagegen über ca. $2 \mathrm{~nm}$ deutlich schärfer. Zu beachten ist auch die nichtverschwindene Nb-Konzentration in der Pd-Schicht von nahezu 10 at.\%. Da in der TAP-Analyse keine Durchmischungseffekte auftreten, lassen sich die beobachteten Durchmischungen auf den Prozeß der Laserdeposition zurückführen. Dabei 


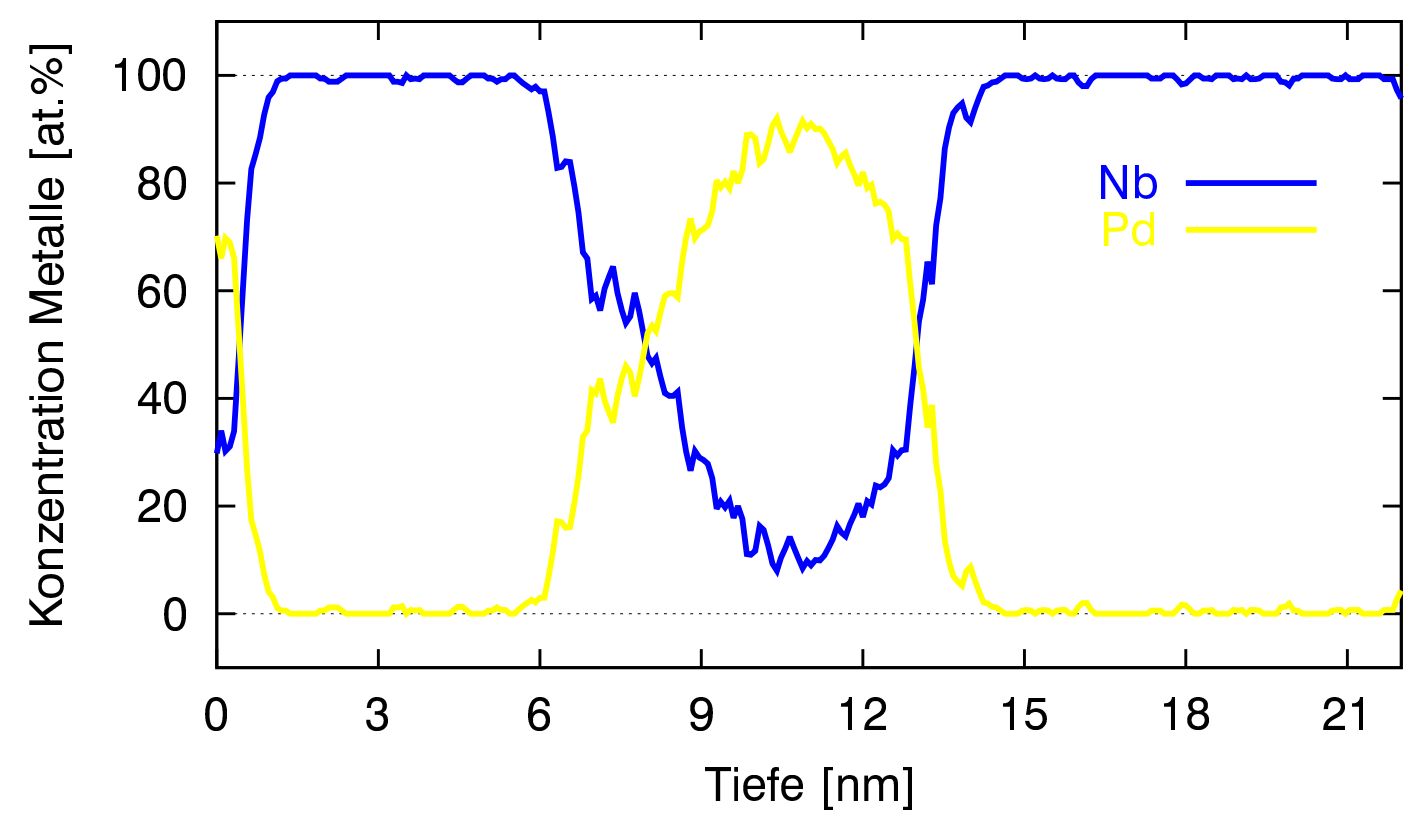

Abbildung 4.12: Konzentrationsprofil der Messung der $2 \mathrm{Pd} / \mathrm{Nb}$ Doppelschichten $\mathrm{Pd} / \mathrm{Nb}$ bei $60 \mathrm{~K}$ (vgl. Abb. 4.11). Gemessen wurde von links nach rechts. Aufgetragen sind die Konzentrationen von $\mathrm{Nb}$ und $\mathrm{Pd}$ (Analysevolumen 0,24 $\mathrm{nm} \times 28,3 \mathrm{~nm}^{2}$ ). Die linke Grenzfläche der mittleren Pd-Schicht zeigt mit ca. $4 \mathrm{~nm}$ eine breitere Interdiffusionszone als die an der rechten Grenzfläche von ca. $2 \mathrm{~nm}$.

erkennt man, daß die Deposition von $\mathrm{Pd}_{\text {auf }} \mathrm{Nb}^{1}$ zu Durchmischungen von ca. 2 $\mathrm{nm}$ Breite führt, während die Deposition von $\mathrm{Nb}$ auf $\mathrm{Pd}$ zu einer Durchmischung von ca. $4 \mathrm{~nm}$ Breite führt. Zusätzlich bildet sich hier schon die PdNb-Phase auf einer Breite von ca. $1 \mathrm{~nm}$. Die hohen kinetischen Energien der deponierten Teilchen von über 100 eV [52] bei der Laserdeposition führen demnach zu Durchmischungseffkten. Dabei dringt das Nb stärker in die Pd-Schicht ein, als im umgekehrten Fall.

Bei den SIMS-Messungen (Abb. 4.9 und 4.10) ist die Bildung der NbPdPhase nicht so deutlich zu erkennen, dafür jedoch an allen Grenzflächen, d.h. auch an der hinteren $\mathrm{Pd} / \mathrm{Nb}-$ Grenzfläche der laserdeponierten Schicht und an beiden Grenzflächen der gesputterten Schicht. Die Bildung einer NbPd-Phase an der hinteren Pd/Nb-Grenzfläche der laserdeponierten Schicht kann mit dem TAPErgebnis (Abb. 4.12) nicht bestätigt werden, hier zeigt sich ein deutlich steilerer Abfall der Pd-Konzentration.

Bei den gesputterten Schichten, bei denen die geringe Depositionsenergie $(<10$ eV) zu schärferen Grenzflächen führen sollte, ist eine solch starke Durchmischung wie bei den laserdeponierten Schichten ebenfalls nicht zu erwarten. Dies spricht

\footnotetext{
${ }^{1}$ Die Schichten bei geringeren Meßtiefen wurden zuletzt aufgebracht.
} 
für eine Phasenbildung im Nb/Pd-System während der SIMS-Messung auf Grund von Durchmischungseffekten durch den Ionenbeschuß.

Um diese Annahme zu bestätigen wurden TAP-Untersuchungen an gesputterten Nb/Pd-Systemen durchgeführt. Der direkte Vergleich mit einer TAP-Analyse einer gesputterten $\mathrm{Nb} / \mathrm{Pd}$-Grenzfläche war jedoch leider nicht möglich, da die entsprechenden Systeme im TAP nicht die nötige Stabilität zeigten. Die hohe Fehlpassung der Gitter führte bei allen Analysen zum Abreißen der Schichten an den $\mathrm{Pd} / \mathrm{Nb}-$ Grenzflächen. Dies ist ein indirekter Hinweis auf eine geringere Durchmischung an den Pd/Nb-Grenzflächen, da eine stärkere Durchmischung die mechanische Stabilität der Grenzflächen erhöht.

Einen weiteren indirekten Hinweis auf den starken Durchmischungseffekt der Laserdeposition können die im folgenden beschriebenen TAP-Messungen an Fe/VSchichten geben, die mit der Sputterdeposition hergestellt wurden und erfolgreich mit dem TAP gemessen werden konnten. So gelingt zumindest der Vergleich mit gesputterten Grenzflächen, auch wenn sich durch den Wechsel der Metallkombination die Ergebnisse nicht direkt übertragen lassen.

\subsubsection{Das System Fe/V}

Die Fe/V-Schichtsysteme wurden alle mit der Sputterdeposition hergestellt, und zwar wie in Abschnitt 4.1.2 beschrieben an zwei unterschiedlichen Anlagen. Eine erlaubte die Deposition bei hohen Temperaturen, die zweite die Reinigung der Substrate im Ar-Ionen-Strahl.

Abb. 4.13 zeigt die Rekonstruktion eines zweifachen Doppelschichtsystems aus $\mathrm{V} /$ Fe-Schichten auf W mit einer Deckschicht Pd. Im W lassen sich die Gitterebenen erkennen. Das Volumen wurde mit einem mittlern Atomvolumen von $13,2^{3}$ rekonstruiert und beträgt 7,5 x 7,5 x $43 \mathrm{~nm}^{3}$. Der eingezeichnete Zylinder zur Konzentrationsanalyse hat einen Radius von 2,5 $\mathrm{nm}$.

Die Konzentrationswerte (Abb. 4.14) wurden in einem Analysevolumen der Tiefe 0,24 nm bestimmt. Das Analysevolumen wurde schrittweise um 0,08 nm verschoben, die mittlere Anzahl der Atome im Analysevolumen war dabei jeweils größer als 100. Man erkennt im Konzentrationsprofil je nach Metallkombination unterschiedliche Grenzflächenschärfen. An der Fe/W-Grenzfläche liegt die gemessene Durchmischung unter 0,5 nm, wogegen sie an den Fe/V- und der $\mathrm{Pd} / \mathrm{V}$-Grenzfläche bis zu $2 \mathrm{~nm}$ groß wird. Diese Durchmischung ist aber eine obere Grenze, die durch die Art der Konzentrationsanalyse beschränkt ist. Wie in Abschnitt 3.1.2 angesprochen, muß man bei der Konzentrationsanalyse zwischen hoher Ortsauflösung, d.h. kleinem Analysevolumen, und guter chemischer Auflösung, d.h. großer Anzahl von Atomen im Analysevolumen, abwägen.

Um eine möglichst große Tiefenauflösung im Bereich der Grenzflächen zu erreichen, wurde eine geringe Tiefe des Analysevolumens gewählt $(0,24 \mathrm{~nm})$. Um dennoch eine Zahl von über 100 detektierten Ionen ins Analysevolumen zu bekommen, wird dafür die laterale Ausdehnung, d.h. der Zylinderradius, relativ 


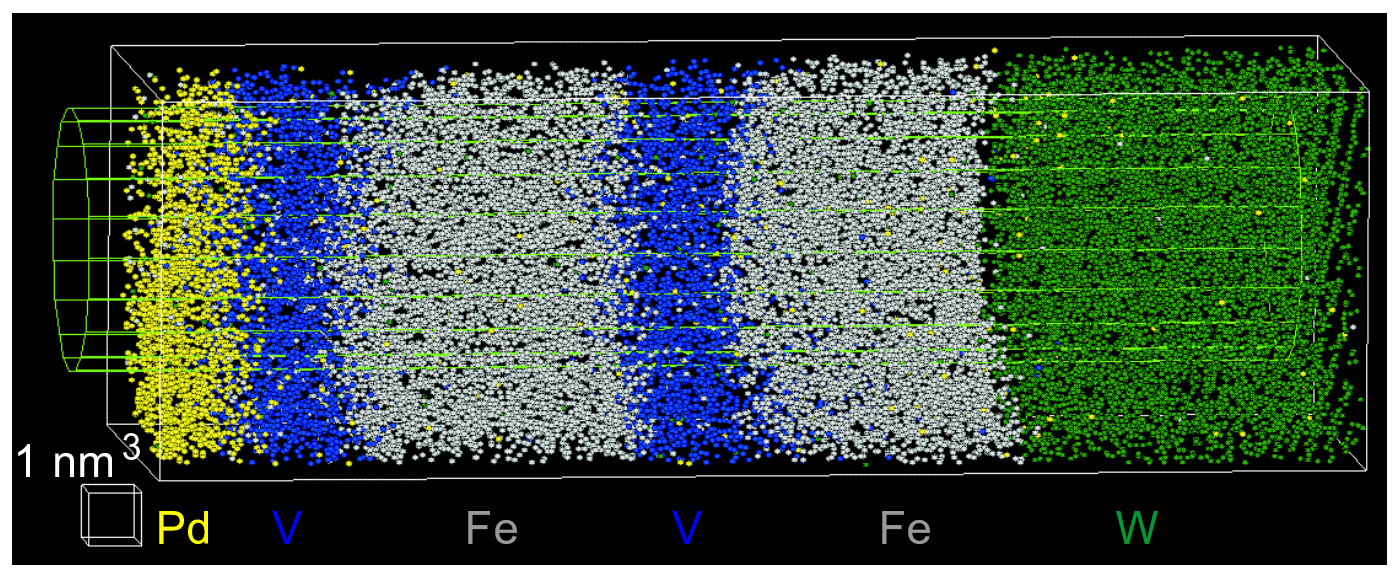

Abbildung 4.13: Rekonstruierte V/Fe-Doppelschichten. Hergestellt bei RT, gemessen im Ausgangszustand bei $60 \mathrm{~K}$. Dargestellt sind folgende Atomsorten: Pd (gelb), V (blau), Fe (grau) und $W$ (grün). Das Volumen wurde mit einem mittlern Atomvolumen von $13,2^{3}$ rekonstruiert und beträgt 7,5 $\times 7,5 \times 43 \mathrm{~nm}^{3}$. Der eingezeichnete Zylinder markiert das Teilvolumen, in dem die Konzentrationsanalyse durchgeführt wurde (Abb. 4.14). Er hat einen Radius von 2,5 nm. Im W-Substrat erkennt man die Gitterebenen.

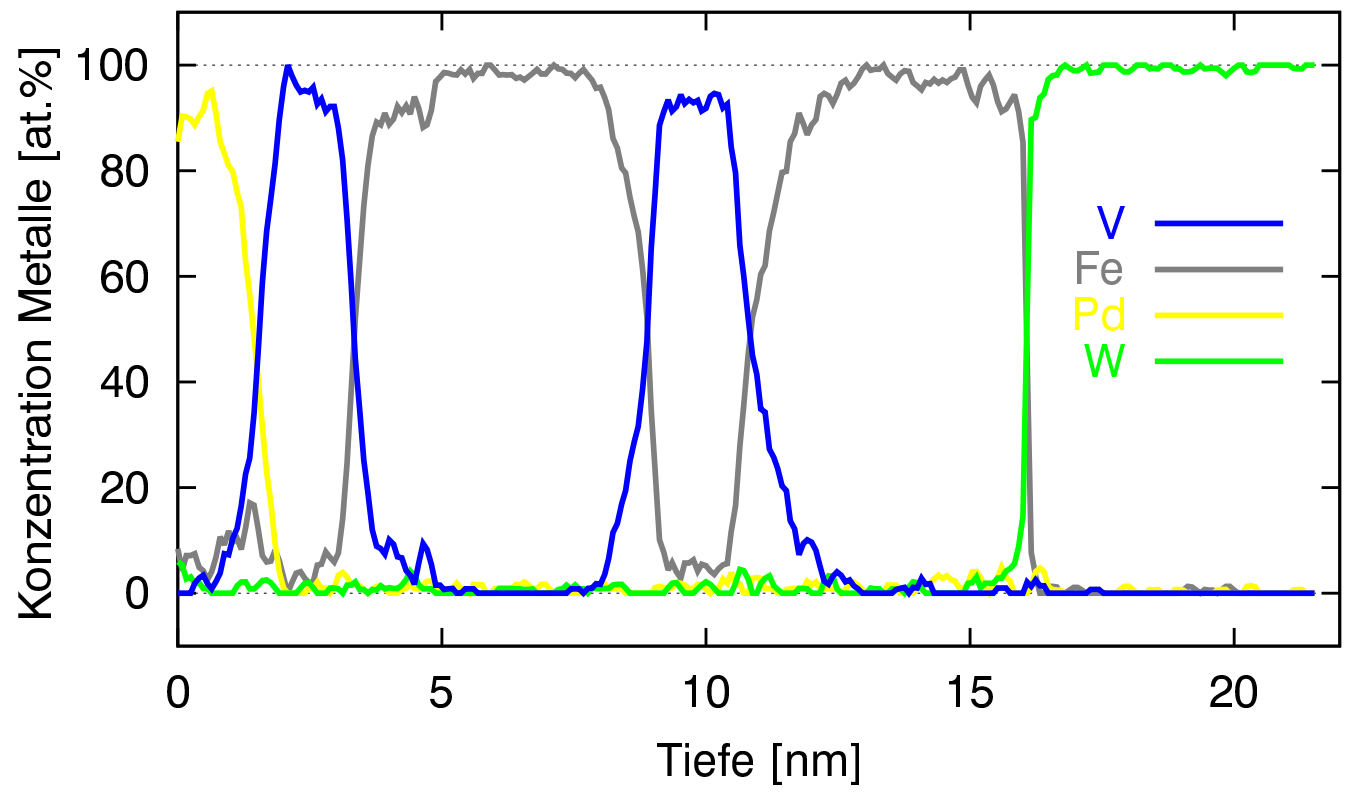

Abbildung 4.14: Konzentrationsprofil der Messung der V/Fe-Doppelschichten bei $60 \mathrm{~K}$ (vgl. Abb. 4.13). Gemessen wurde von links nach rechts. Aufgetragen sind die Konzentrationen von $V, F e, P d$ und $W$ (Analysevolumen: 0,24 nm x 19,6 $\left.n m^{2}\right)$. Die bestimmten Grenzfl̈̈chenschärfen liegen an der Fe/W-Grenzfläche bei $0,5 \mathrm{~nm}$, für $\mathrm{Fe} / \mathrm{V}$ und $\mathrm{Pd} / \mathrm{V}$ bei $2 \mathrm{~nm}$. 
groß gewählt $(2,5 \mathrm{~nm})$. Dieser große Radius führt bei der Konzentrationsanalyse der Grenzflächen, die über das gesamte analysierte Volumen in den seltensten Fällen absolut eben sind, zu einer Überschätzung der Durchmischung an der Grenzfläche. Eine eingehende Diskussion der Fehler der dreidimensionalen Volumenrekonstruktion folgt in Abschnitt 5.7.1.

Die mit dem TAP bestimmten, eher zu großen Werte der Grenzflächendicke liegen immer noch um mehr als einen Faktor 2 unterhalb der Auflösung der SIMS-Analysen. Zudem zeigen die gesputterten Schichten keine Durchmischung auf einer Länge von $4 \mathrm{~nm}$, wie sie am laserdeponierten $\mathrm{Pd} / \mathrm{Nb}$-System gemessen wurde (Abb. 4.12).

Zumindest indirekt läßt sich somit die geringere chemische Grenzflächendurchmischung der gesputterten Schichtsysteme im Vergleich zum laserdeponierten System zeigen. Was die Methoden SIMS und TAP angeht, so zeigt das TAP eindeutig die bessere Tiefenauflösung, da es ohne den die Proben durchmischenden Sputterprozeß auskommt. 


\section{Kapitel 5}

\section{Wasserstoffverteilung in metallischen Multischichten}

Die Ergebnisse der Messungen an H- und D-beladenen Schichtsystemen, die mit den hochauflösenden Analyseverfahren SIMS und TAP gewonnen wurden, werden im folgenden Kapitel beschrieben und diskutiert. Schwerpunkte sind zum einen die Untersuchungen der Rahmenparameter, bei denen eine nanoskalige Analyse der H- bzw. D-Verteilung in Multischichtsystemen mit SIMS und TAP möglich wird. Ein besonders kritischer Parameter ist dabei die Meßtemperatur. Zum anderen werden die bei geeigneten Parametern durchgeführten Untersuchungen zur H-Konzentration und lokalen H-Verteilung in den Schichten dargestellt und analysiert. Dabei liegt das Hauptaugenmerk auf dem Bereich der Grenzflächen, in dem die Korrelation zwischen H- und Metallkonzentrationen, Aussagen über Grenzflächeneffekte der H-Löslichkeit zulassen sollte. Zunächst werden kurz die verwendeten Methoden zur H- bzw. D-Beladung der in Kapitel 4 charakterisierten Schichtsysteme beschrieben.

\section{$5.1 \quad$ H-/D-Beladung}

Die H- bzw. D-Beladung der Schichtsysteme wurde mit zwei unterschiedlichen Verfahren durchgeführt.

\subsubsection{D-Beladung aus der Gasphase}

Die für die Feldionenmikroskopie präparierten Schichtsysteme wurden aus der Gasphase beladen. Da das TAP nur sehr eingeschränkt H-Ionen der Masse $1 \mathrm{amu}$ detektieren kann und zudem Wasserstoff auch als Restgas im Vakuum vorhanden ist, wurden die Schichten mit Deuterium beladen. Dazu wurde eine heizbare Sorptionsapparatur verwendet, die bei Wang [59] beschrieben ist. Die Schichten wurden zunächst im Vakuum bei $200^{\circ} \mathrm{C}$ für ca. $12 \mathrm{~h}$ ausgelagert, um den bei der 
Herstellung eingebrachten Wasserstoff auszugasen. Bei der gewählten Temperatur von $200^{\circ} \mathrm{C}$ ist die Interdiffusion der verwendeten Metalle vernachlässigbar. Anschließend wurden die Schichten bei Raumtemperatur ohne Brechen des Vakuums mit D-Gas beladen. Die verwendeten Drücke lagen im Bereich von 2 - 14 mbar $\mathrm{D}_{2}$ und wurden für Zeiten von 0,5 - $72 \mathrm{~h}$ angelegt. Über Druck, Temperatur und Zeit wurde die D-Konzentration in den Systemen variiert.

\subsubsection{Elektrochemische H-Beladung}

Die Proben für die SIMS-Messungen wurden elektrochemisch mit Wasserstoff beladen. Dazu wird die zu beladene Probenoberfläche als Elektrode in eine elektrochemische Zelle eingebaut. Zwischen einer Gegenelektrode und der Probe wird dann eine Gleichspannung angelegt, wobei die Probe die Kathode bildet. Die Protonen werden an der Pd-Probenoberfläche zu atomarem Wasserstoff entladen, der dann im Metall gelöst wird. Maximale Stromdichten von $5 \mathrm{~mA} / \mathrm{cm}^{2}$ gewährleisten die Aufnahme des gesamten Wasserstoffs, solange die Konzentration im Gitter niedrig ist. Bei Kenntnis der geflossenen Ladung $Q$ läßt sich somit sehr präzise die Menge an aufgenommenem Wasserstoff bestimmen. Unter der Voraussetzung, daß der gesamte Wasserstoff in die Probe eindringt, läßt sich die Anzahl der Mole in der Probe $n_{H_{M e}}$ nach der Beladung ermitteln. Nach dem Gesetz von Faraday benötigt man für die Entladung eines Mols eines einwertigen Ions eine Ladungsmenge $F$

$$
n_{H_{M e}}=\frac{Q}{F} \cdot n_{H^{+}} .
$$

Die Molanzahl von Wasserstoff kann zu der Molanzahl der Metallatome der Probe $n_{M e}$, die man aus dem Probenvolumen $V$ und den molaren Volumina $V_{M}$ der beteiligten Metalle ermittelt, ins Verhältnis gesetzt werden. Als Ergebnis ergibt sich die H-Konzentration

$$
\begin{aligned}
c & =\frac{n_{H_{M e}}}{n_{M e}} \\
& =\frac{Q}{F} / \frac{V}{V_{M}} .
\end{aligned}
$$

Die experimentellen Parameter, die in die Berechnung der Konzentration einfließen, sind somit die Ladung und das Probenvolumen.

\subsection{Messung der H-Verteilung bei $300 \mathrm{~K}$ (SIMS- Analysen)}

Pd/Nb-Schichtpakete aus 2 bis 6 Doppelschichten auf Si mit Doppelschichtdicken von 12 bis $50 \mathrm{~nm}$ und Gesamtdicken bis zu $150 \mathrm{~nm}$ wurden mit dem SIMS untersucht. 

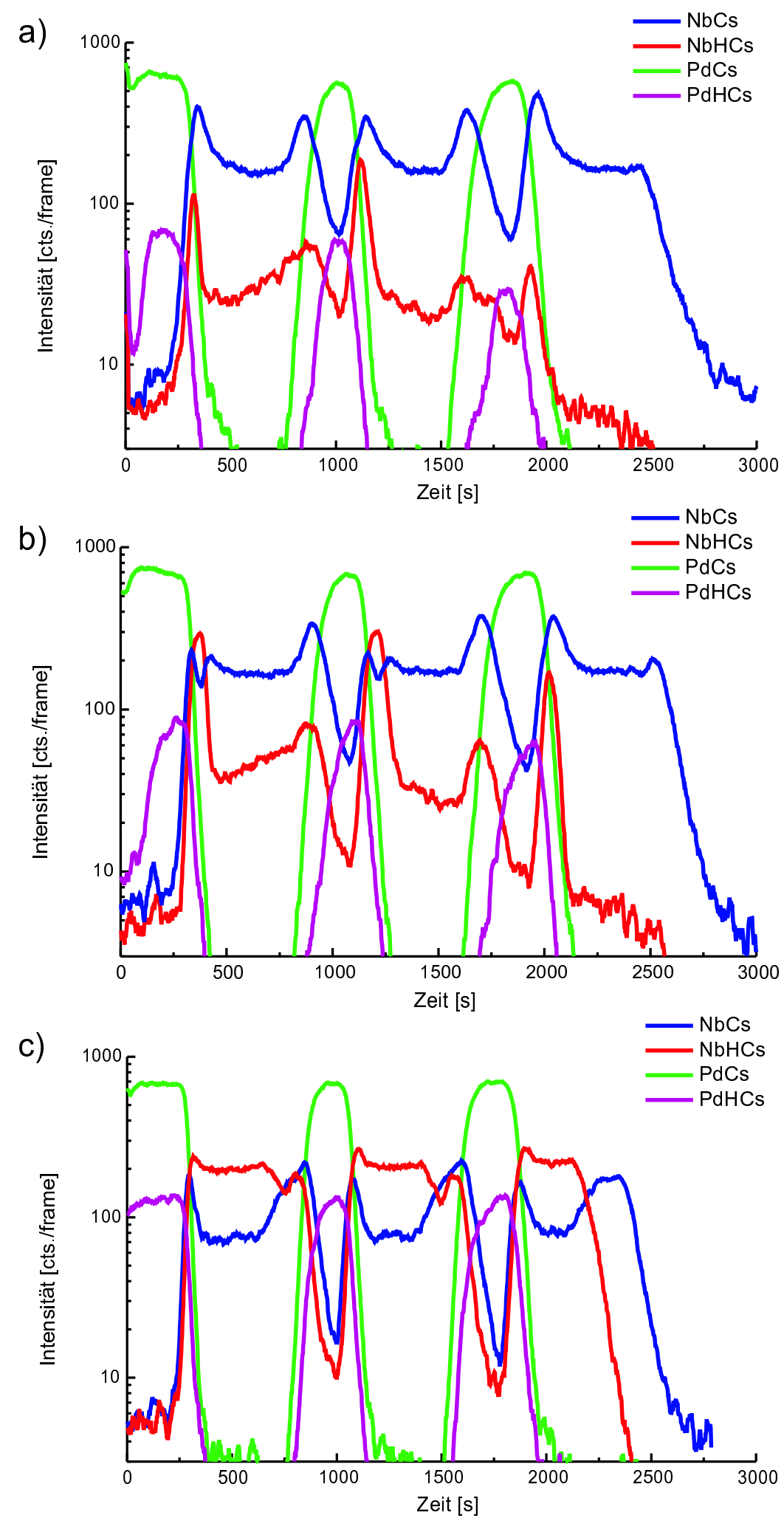

Abbildung 5.1: SIMS-Tiefenprofile einer Pd/Nb-Multischicht aufgenommen nach H-Beladungen von a) $0,04, \boldsymbol{b}) \mathbf{0 , 1 0} \mathrm{H} / \mathrm{Nb}$ und c) vollständiger Beladung. Das System besteht aus 3 Doppellagen mit Einzelschichtdicken von jeweils $25 \mathrm{~nm}$. Aufgetragen sind die Zählraten von PdCs, NbCs, NbHCs, Si und PdHCs. Analysiert wurde auf einer Fläche von (50 x 50) $\mu^{2}$ mit Ga-Ionen. Zum Tiefenabtrag wurde mit Cs-Ionen ein Krater von (200 x 200) $\mu^{2}$ gesputtert. 
Die Schichtsysteme wurden nach den Messungen des Ausgangszustands (beschrieben in Abschnitt 4.3.1) außerhalb des SIMS elektrochemisch mit Wasserstoff beladen und erneut eingebaut und gemessen. Die Meßreihe Abb. 5.1 zeigt typische SIMS-Tiefenprofile eines Schichtpaketes von 3 Doppelschichten Pd/Nb mit Einzelschichtdicken von $25 \mathrm{~nm}$. Das System wurde nacheinander mit 3 unterschiedlichen H-Konzentrationen beladen. Bei einer Beladung sollte durch den hohen Löslichkeitsunterschied zwischen $\mathrm{Nb}$ und $\mathrm{Pd}$ zunächst sämtlicher Wasserstoff im Nb gelöst werden (s.Tab. 2.8). Deswegen sind die folgenden Konzentrationsangaben auf die Nb-Schichten bezogen. Die aus der Ladungsmenge der elektrochemischen Beladung berechneten Konzentrationen betrugen 0,04, 0,1 und 10 H/Nb. Die gewählten Werte entsprechen Konzentrationen aus der $\alpha$-Phase $(0,04 \mathrm{H} / \mathrm{Nb})$, aus dem Zweiphasengebiet $(0,10 \mathrm{H} / \mathrm{Nb})$ und der $\beta$-Phase des Phasendiagramms für das massive Nb-H-System (Abb. 2.3). Die Beladung von $10 \mathrm{H} / \mathrm{Nb}$ entspricht einer vollständigen H-Beladung des Schichtsystems, bei der sowohl das Nb als auch das Pd hydriert wird und überschüssiger Wasserstoff unter $\mathrm{H}_{2}$-Gasentwicklung in den Elektrolyten entweicht. Messungen von Laudahn [7] haben gezeigt, daß dieser Beladungszustand bei Raumtemperatur nicht stabil ist. Der Wasserstoff entweicht innerhalb von Minuten aus den Pd-Schichten. Auch die Nb-Schichten verlieren Wasserstoff, wobei sich jedoch die H-Konzentration am oberen Ende des $\mathrm{Nb}-\mathrm{H}-\mathrm{Z}$ weiphasengebietes stabilisiert. Dies liegt in massivem $\mathrm{Nb}$ bei $0,7 \mathrm{H} / \mathrm{Nb}$. In Nb-Schichten von $50 \mathrm{~nm}$ Dicke wurden jedoch reduzierte Konzentrationen kleiner als $0,5 \mathrm{H} / \mathrm{Nb}$ beobachtet. Für das untersuchte Schichtsystem werden somit $\mathrm{H}$-Konzentrationen unterhalb von $0,5 \mathrm{H} / \mathrm{Nb}$ erwartet. Vor jeder neuen Beladung wurde die Probe unter Ar-Atmosphäre bei $200^{\circ} \mathrm{C}$ für mindestens $8 \mathrm{~h}$ ausgelagert, um den bereits gelösten Wasserstoff wieder zu entfernen. Die Zeit zwischen Beladung und Messung lag bei ca. $2 \mathrm{~h}$. Abbildung 5.1 zeigt auch, daß sich die $\mathrm{Nb} / \mathrm{Pd}$-Schichten durch diese Behandlung nicht verändern. Es wird auf der Meßskala keine Interdiffusion beobachtet.

Alle Tiefenprofile zeigen bezüglich des H-Signals im $\mathrm{Nb}^{1}$ eine charakteristische Gemeinsamkeit. Das H-Signal variiert sehr stark, jedoch nicht über die gesamte Probe gesehen, sondern vielmehr in jeder einzelnen Nb-Schicht. Das Maximum des H-Signals innerhalb einer Nb-Schicht liegt stets bei kurzen Meßzeiten, d.h. an der Oberseite der beim Meßprozeß abgetragenen Schicht. Es fällt in der Regel schnell ab. Für hohe Konzentrationen kommt es lediglich zu einer Verbreiterung dieses hohen Anfangsniveaus, keine Schicht zeigt jedoch ein konstantes H-Signal innerhalb einer Nb-Lage. Hierauf wird später noch genauer eingegangen. Es stellen sich somit folgende Fragen:

- Sind die Intensitäten bezüglich der H-Konzentration in den Schichten quantitativ auswertbar?

- Gibt das gemessene H-Signal die H-Verteilung in den Schichtsystemen nach

\footnotetext{
${ }^{1}$ Wie in Abschnitt 3.2 beschrieben, wird der Wasserstoff als NbHCs gemessen.
} 
der Beladung wieder, oder hat man es mit Nichtgleichgewichtsverteilungen zu tun, die durch die SIMS-Tiefenprofilierung verursacht werden ?

Der ersten Frage soll zunächst nachgegangen werden. Unter der Annahme, daß bei der Detektion der Cäside die Zählraten elementunabhängig sind, können aus der Zählrate Konzentrationen gewonnen werden, insbesondere die des Wasserstoffs. Hierzu wird der Quotient aus H- und Metallsignalen gebildet. Dabei ist zu berücksichtigen, daß als H-Signal das Metallhydridsignal, d.h. NbHCs und PdHCs gezählt wird, das dementsprechend auch dem Metall zugeschlagen werden muß. Diese berechnete,"momentane" H-Konzentration beim Abtrag der Probe ist für die Messungen aus Abb. 5.1 in Abb. 5.2 zusammengefaßt. Die Konzentration ist in $\mathrm{H} / \mathrm{Me}($ tall) angegeben. Die senkrechten Linien markieren die Zeiten, bei denen das Signal des PdCs auf die Hälfte abgesunken ist. Sie wurden zur Markierung der Grenzflächen gewählt. Die Werte der Messung Abb. 5.1 b), die ca. $6 \%$ langsamer war, sind in Abb. 5.2 um diesen Faktor korrigiert, d.h. leicht gestaucht in der Zeitachse, eingezeichnet. Man erkennt innerhalb der Nb-Schichten mit

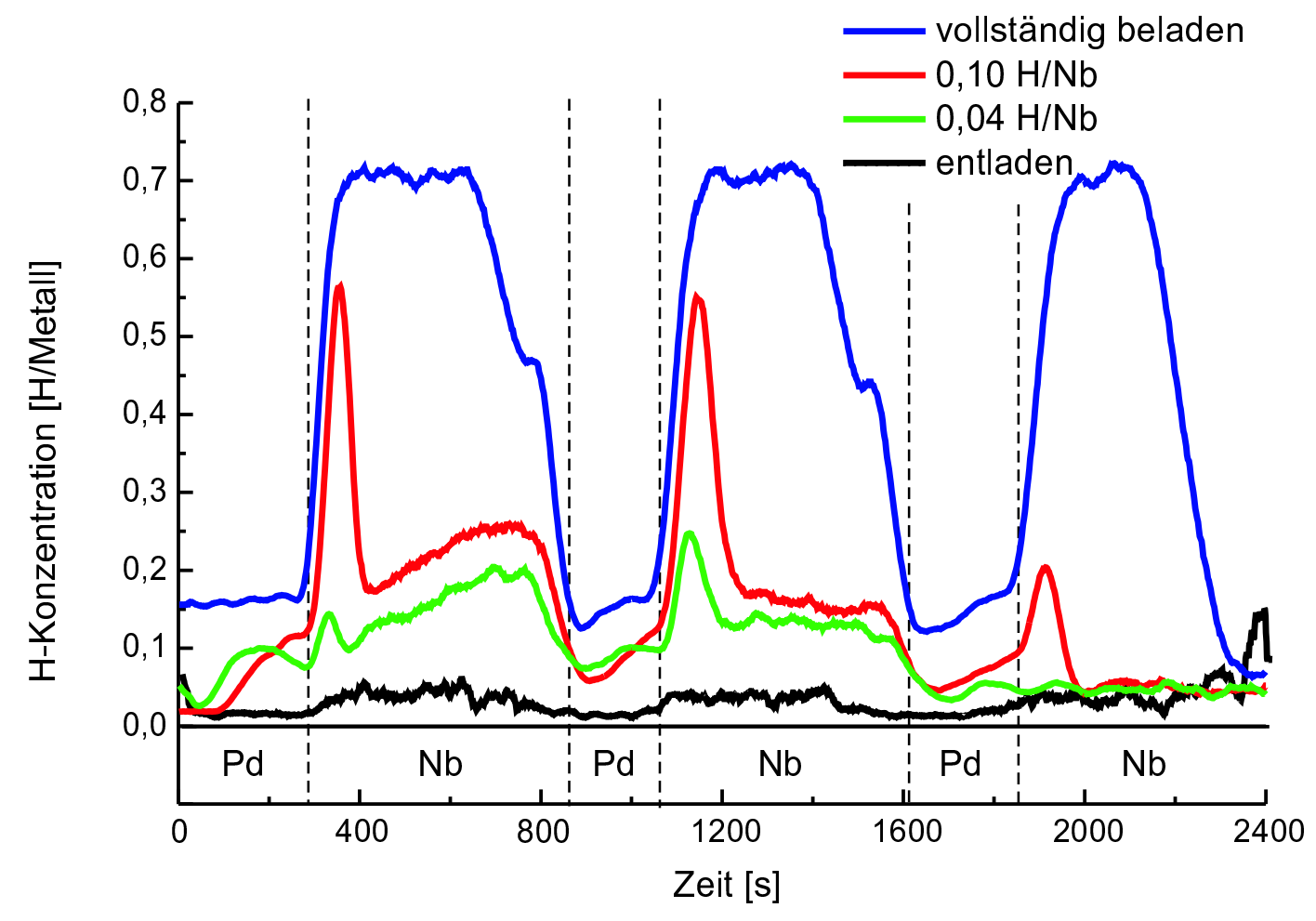

Abbildung 5.2: Aus den SIMS-Messungen (Abbn.5.1a)-c)) berechnete $H$ Konzentrationen (H-/Metall-Signale). Die Kurve der Beladung mit 0,10 H/Nb ist in der Zeitachse um 6\% verkleinert. Eingezeichnet ist ebenfalls die Messung des Schichtsystems im entladenen Zustand.

vollständiger H-Beladung eine Konzentration von ca. 0,7 H/Me, die sich in allen 
drei Nb-Schichten über weite Bereiche der Nb-Schichten ausdehnt. Es verändert sich nur die Breite dieses Niveaus in den drei Schichten. Von der ersten Schicht bis zur letzten Nb-Schicht nimmt die Ausdehnung kontinuierlich ab. Die Messungen mit den geringeren mittleren Konzentrationen zeigen nur einen schmalen Anfangspeak, der im Falle der zehnprozentigen Beladung mit Werten bis zu 0,55 $\mathrm{H} /$ Me deutlich höher ausfällt, als das Maximum von $0,25 \mathrm{H} / \mathrm{Me}$ bei der geringsten Ausgangskonzentration, das erst in der zweiten Nb-Schicht erreicht wird. Auffällig ist zudem, daß die Peaks in der dritten Nb-Schicht jeweils deutlich kleiner ausfallen und daß in der ersten Nb-Schicht nach dem Anfangspeak ein deutlicher Anstieg der Konzentration beobachtet wird.

Auch innerhalb der Pd-Schichten erkennt man ansteigende H-Konzentrationsprofile mit maximalen Werten von $0,17 \mathrm{H} / \mathrm{Me}$ bei der vollständig beladenen Messung und 0,12 H/Me bzw. 0,10 H/Me bei den geringeren Beladungen. Eingezeichnet ist außerdem die Kurve des entladenen Systems. Die Untergrundkonzentration liegt bei 0,02 H/Me im Pd und bis zu 0,04 H/Me im Nb. Die Konzentrationen sind alle in $\mathrm{H} / \mathrm{Me}$ angegeben, im Inneren der Schichten lassen sie sich auch jeweils als $\mathrm{H} / \mathrm{Nb}$ bzw. H/Pd deuten, da hier die Zählraten des jeweils anderen Metalls vernachlässigbar sind. An den Grenzflächen würden die nur auf ein Schichtelement bezogenen Konzentrationen jedoch signifikant höher liegen. So fallen die Anfangspeaks in den gering beladenen Messungen um rund $20 \%$ höher aus, wenn man sie nur auf das Nb bezieht. Da durch die SIMS-Analyse die Metalle an den Grenzflächen auf einer Skala von wenigen nm durchmischt werden (vgl. Abschnitt 4.3.1), ist es gerechtfertigt, die H-Konzentration jeweils auf die Summe von Pd und Nb zu beziehen.

Mittelt man über die Konzentration innerhalb der Nb-Schichten, so erhält man die in Tab. 5.1 angegebenen Konzentrationen, bei denen der Untergrund von 0,04 $\mathrm{H} / \mathrm{Me}$ bereits abgezogen wurde. Für die vollständige Beladung wurde als Ausgangskonzentration der erwartete Wert von $0,5 \mathrm{H} / \mathrm{Nb}$ eingesetzt. Man erkennt, daß die gemittelten Werte die nominellen Beladungswerte recht gut wiedergeben. Der Fehler des nominellen Wertes, d.h. der Beladung, liegt unterhalb von 20\% des angegebenen Wertes, der sich hauptsächlich aus dem Fehler der Schichtdickenbestimmung und in kleinerem Maße auch aus dem Fehler im Beladungsstrom und der elektrochemischen Aufnahme des Wasserstoff zusammensetzt. Die Abweichung der mittleren Konzentration in den Nb-Schichten von der nominellen liegt deutlich unterhalb eines Faktors 2. Die Ergebnisse der Messungen mit geringeren Konzentrationen deuten ferner auf eine systematische Überschätzung der H-Konzentration, was auf eine höhere Detektionswahrscheinlichkeit der Metallhydridionen im Vergleich zu den Metallionen hinweist.

Nach diesen Ergebnissen wird der Wasserstoff in der jeweils ersten Schicht in höheren Konzentrationen gemessen als in den folgenden. Sie deuten auf dynamische Prozesse hin, womit wir bei der Beantwortung der zweiten Frage angelangt sind.

Für die Pd-Schichten erwartet man außer im Fall der vollbeladenen Nb- 
Schichten ein verschwindendes H-Signal, denn $\mathrm{Nb}$ sollte im Vergleich zum $\mathrm{Pd}$ den Wasserstoff in einem Verhältnis von $3 \cdot 10^{4}$ und somit nahezu komplett lösen (s.Tab. 2.8). Jedoch findet man in den Messungen mit geringer Beladung untergrundbereinigte Konzentrationen von bis zu 0,10 H/Pd und in der Messung der vollbeladenen Multischicht ausschließlich Werte größer als 0,14 H/Pd. Damit lägen die gefundenen Konzentrationen im Zweiphasengebiet des massiven Pd-HSystems. Röntgenmessungenan an vergleichbaren Multischichtsystemen [7] haben aber ergeben, daß dies kein stabiler Zustand des Systems ist. Das ist ein weiterer Hinweis darauf, daß das SIMS-Profil nicht den Gleichgewichtszustand wiedergibt.

Um den Nichtgleichgewichtszustand näher zu untersuchen, wurden Messungen mit unterschiedlichen Abtragsraten durchgeführt. Durch die damit verbundene Veränderung der Meßzeit sollte geklärt werden, ob während der Messung Diffusionsprozesse auftreten. Es wurde ein Schichtpaket mit 6 Doppellagen $\mathrm{Pd} / \mathrm{Nb}$ und Einzelschichtdicken von $12,5 \mathrm{~nm}$ mit $0,10 \mathrm{H} / \mathrm{Nb}$ beladen und mit unterschiedlichen Geschwindigkeiten im SIMS gemessen. Die Abtragsgeschwindigkeit in die Tiefe läßt sich durch Variation der Fläche erreichen, auf der der Cs-Krater gesputtert wird. So wird bei größerem Krater die deponierte Energie pro Fläche kleiner und somit auch die Abtragsgeschwindigkeit. Abbildung 5.3 zeigt die Tiefenprofile der Pd/Nb-Multischicht. Man erkennt, daß durch die Vergrößerung des Cs-Kraters die Meßzeit für das gesamte Schichtpaket stark zunimmt, von ca. $3500 \mathrm{~s}$ (a) auf ca. $30000 \mathrm{~s}$ (b) (Faktor 8,85). Die Fläche des Kraters nimmt von $(200 \times 200) \mu m^{2}$ auf $(500 \times 500) \mu m^{2}$ um einen Faktor 6,25 zu.

In Abb. 5.4 ist die aus den Profilen berechnete H-Konzentration bei schneller Messung der bei langsamer gegenübergestellt. Dabei wurde die langsame Messung auf die Messzeit der schnelleren herunterskaliert (Faktor 1/8,85). Eingezeichnet ist auch jeweils die mittlere Konzentration innerhalb der Nb-Schichten. Es zeigt sich eine deutliche Abhängigkeit der H-Konzentrationen von der Meßgeschwindigkeit. Auffälligster Unterschied ist die erhöhte H-Konzentration bei langsamer Messung in den ersten Schichten. Während in der schnellen Messung die mittlere Konzentration verhältnismäßig konstant bleibt und erst in den letzten beiden Schichten abfällt, erkennt man in der langsamen Messung ausgehend von einem hohen Wert in der ersten Schicht einen gleichmäßigen Abfall von Schicht zu Schicht. Dabei sinken die mittleren Werte ab Schicht 4 unter die Werte der schnellen Messung. Die berechneten mittleren Gesamtkonzentrationen von 0,11 H/Nb (schnelle Messung)

\begin{tabular}{|c||c|c|c||c|}
\hline \multicolumn{1}{|c||}{$\mathrm{H} / \mathrm{Nb}$} & \multicolumn{4}{c|}{$\mathrm{H} / \mathrm{Me}$ (SIMS) } \\
nominell & Schicht 1 & Schicht 2 & Schicht 3 & $\overline{\mathrm{c}}$ \\
\hline 0,50 & 0,46 & 0,44 & 0,44 & 0,44 \\
0,10 & 0,19 & 0,18 & 0,07 & 0,15 \\
0,04 & 0,09 & 0,10 & 0,01 & 0,07 \\
\hline
\end{tabular}

Tabelle 5.1: Mittlere H-Konzentrationen für die Nb-Schichten, berechnet aus den Kurven der Abb. 5.2. 

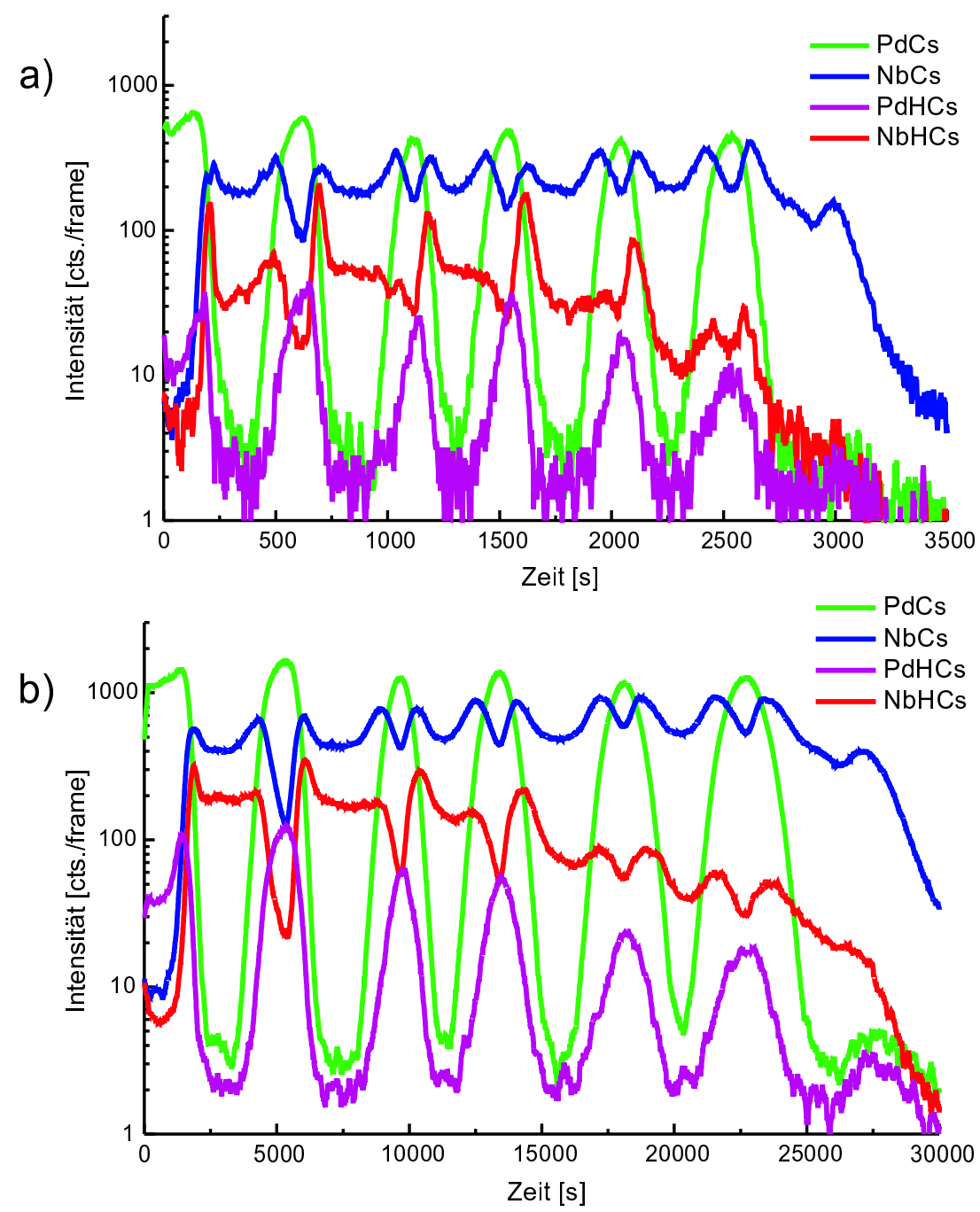

Abbildung 5.3: SIMS-Tiefenprofil einer Pd/Nb-Multischicht aus 6 Doppellagen mit Einzelschichtdicken von jeweils 12,5 nm auf Si. Die Schicht war mit 0,10 $\mathrm{H} / \mathrm{Nb}$ beladen. a) Analysiert wurde auf einer Fläche von (50 x 50) $\mu^{2}$ mit GaIonen. Zum Tiefenabtrag wurde mit Cs-Ionen ein Krater von (200 x 200) $\mu^{2}$ gesputtert. b) Analysiert wurde auf einer Fläche von (300x 300) $\mu^{2}$ mit GaIonen. Zum Tiefenabtrag wurde mit Cs-Ionen ein Krater von (500 x 500) $\mu^{2}$ gesputtert.

und 0,12 H/Nb (langsame Messung) unterscheiden sich innerhalb des Fehlers aus der Berechnung von ca. $20 \%$ nicht.

Betrachtet man die Konzentrationsverläufe innerhalb der einzelnen Nb-Schichten, so erkennt man nur in der schnellen Messung die schon in Abb. 5.2 beschriebenen Konzentrationspeaks am Anfang der Nb-Schichten. Der Vergleich der beiden Kurven zeigt somit für die langsame Messung eine Anreicherung des Wasserstoff 


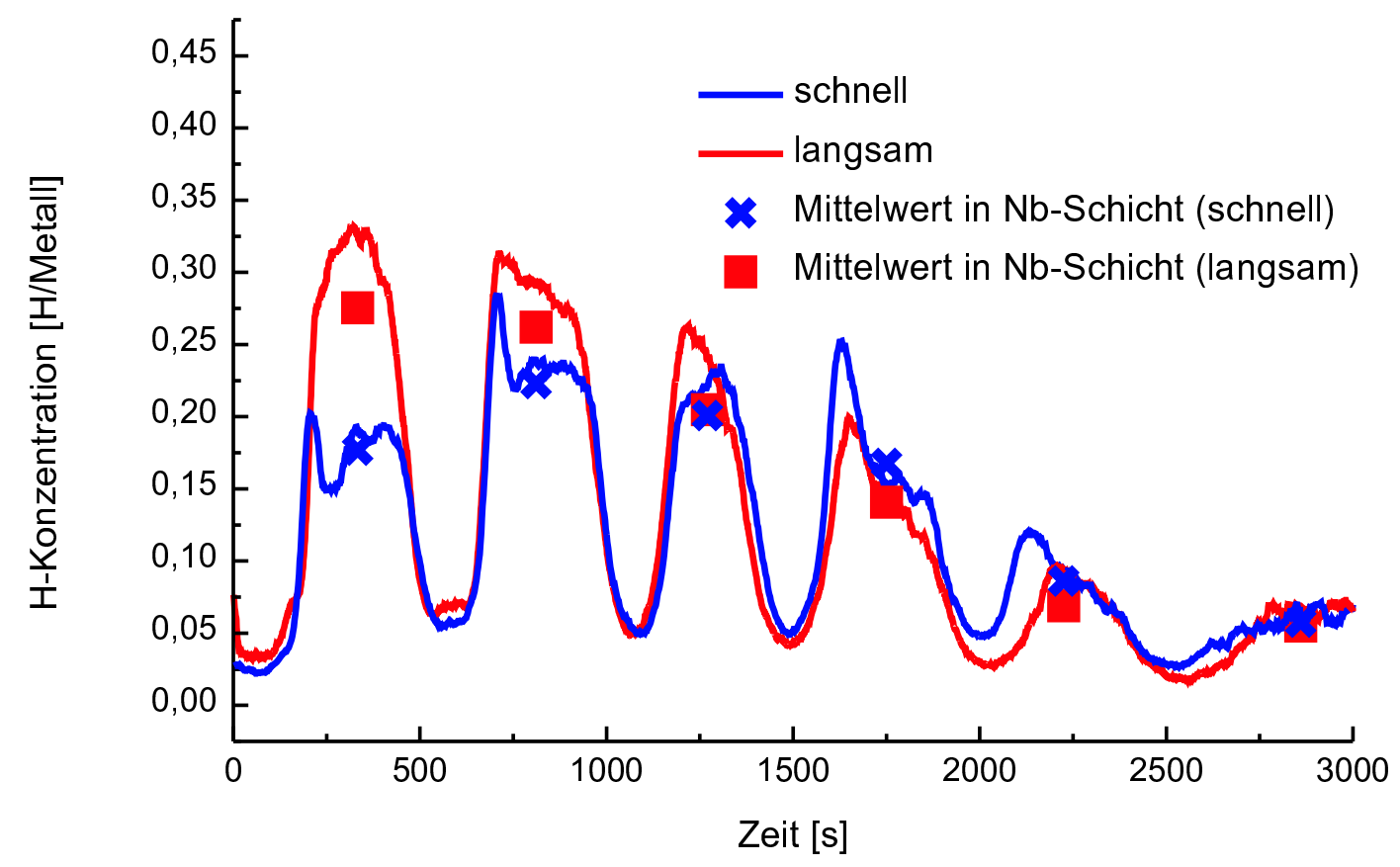

Abbildung 5.4: Berechnete H-Konzentrationen einer schnellen und einer langsamen SIMS-Messung (Abb. 5.3). Das System besteht aus 6 Doppellagen mit Einzelschichtdicken von jeweils 12,5 $\mathrm{nm}$ und war mit 0,10 H/Nb beladen. Die Signale der langsamen Messung wurden an Hand der PdCs-Signale auf die schnelle Messung herunterskaliert (Faktor 1/8,85 in der Zeit). Als Punkte eingezeichnet sind die mittleren H-Konzentrationen innerhalb einer Nb-Schicht. Man erkennt eine deutliche Abhängigkeit der H-Konzentrationen von der Meßgeschwindigkeit.

in den ersten Nb-Schichten bei gleichzeitiger Verarmung in den hinteren Schichten. Dies spricht für einen Diffusionsprozeß, denn bei langsamerer Messung hat der Wasserstoff mehr Zeit, an die momentane Oberfläche zu diffundieren und dort die H-Konzentration hoch zu halten. Daß die H-Konzentrationen in den tieferen Schichten immer mehr abnehmen, spricht für eine Diffusion aus den tieferen NbLagen durch die als Diffusionsbarriere wirkenden Pd-Lagen hindurch. Der Diffusionsprozeß und der Ursprung der für die Diffusion nötigen treibenden Kraft wird im folgenden Abschnitt diskutiert.

\subsection{Diskussion der Ergebnisse der Messungen bei $300 \mathrm{~K}$}

Die Tiefenprofilierung des SIMS (beschrieben in Abschnitt 3.2) beruht auf einer Sequenz von Oberflächenmessungen mit zwischengeschalteten Abtragsschritten. Die gemessenen Signale bilden somit stets die aktuelle Oberfläche ab. Aus dieser 
Sequenz von Oberflächenmessungen quantitative Konzentrationsprofile zu gewinnen, ist eine Hauptschwierigkeit der SIMS-Analyse. Ausgehend von den Arbeiten von Gnaser et al. [49, 50], die quantitative Konzentrationsanalysen der SIMSProfile mit Cs-Komplexen durchführten, sind am Institut Arbeiten zur Quantifizierung der Daten von Bankmann [51] und Hartung [60] durchgeführt worden. Bei den durchgeführten Messungen in dieser Arbeit tritt als zusätzliche Schwierigkeit nun noch die hohe Beweglichkeit einer Komponente hinzu. Mit den in Tab. 2.2 aufgeführten Diffusionskoeffizienten kann bei $300 \mathrm{~K}$ in einer Sekunde mit Diffusionslängen

$$
x \propto \sqrt{D \cdot t}
$$

des Wasserstoff in den untersuchten Pd- und Nb-Schichten von $6 \mu \mathrm{m}$ im Pd und $3 \mathrm{~mm}$ im Nb gerechnet werden. Während der Meßzeit von einigen hundert bis einigen tausend Sekunden werden somit Diffusionslängen erreicht, die die Schichtdicke deutlich übersteigen. Zur Diffusion kommt es jedoch nur, wenn entsprechende Gradienten im chemischen Potential - hier Konzentrationsgradienten in der Probe vorliegen. Die inhomogenen H-Konzentrationsverläufe der SIMSMessungen zeigen, daß dies während der Messung der Fall ist. Man kann deshalb von H-Diffusionprozessen während der Messung ausgehen. Eine Untersuchung der Gleichgewichtsverteilung ist somit im SIMS bei Raumtemperatur nicht möglich. Interessant sind die Ergebnisse jedoch für die Untersuchung der H-Diffusion in metallischen Multischichten. Deswegen soll im folgenden ein Modell zur Beschreibung der Transportvorgänge in den Multischichten während der SIMS-Messung vorgestellt werden. Daraus abgeschätzte Diffusionskoeffizienten sollen anschliessend mit dem Modell zur H-Permeation durch Multischichten von Schmitz et al. [61] verglichen werden.

\subsubsection{Einfluß der H-Diffusion}

Das hohe H-Signal an der Oberflächenseite jeder Nb-Schicht bedeutet, daß der Wasserstoff beim Absputtern der Nb-Oberfläche zunächst in hohen Konzentrationen vorliegt und dann erst nach dem Abtrag einer gewissen Schichtdicke abfällt. Dies läßt sich erklären, wenn man annimmt, daß der zunächst gleichmäßig in der Schicht verteilte $\mathrm{H}$, sobald die Nb-Oberfläche freiliegt, an diese diffundiert, da er hier energetisch günstigere Plätze findet und mit Konzentrationen von bis zu 1 $\mathrm{H} / \mathrm{Nb}$ segregieren kann [62]. Im nachfolgenden Sputterzyklus wird diese Oberfläche oder zumindest Teile der Segregationszone samt Wasserstoff abgetragen und es bieten sich "frische" Oberflächenplätze. Das H-Messsignal wird dann solange erhöht detektiert, bis der Nachfluß des Wasserstoff aus der Nb-Schicht bzw. dem dahinterliegenden Schichtsystem versiegt. Eine alternative Deutung dieses Effektes liefern Züchner et al. [26], die eine erhöhte oberflächennahe Segregation mit durch den Sputterprozeß eingebrachten Defekten erklären. Die Frage, ob die Bildung von frischen Oberflächenplätzen die Ursache der H-Konzentrationsgradienten ist, oder 
die durch den Ionenbeschuß unterhalb der Oberfläche eingebrachten Defekte, wie von Züchner et al. vorgeschlagen, wird in Rahmen der Diskussion (Abschnitt 5.6) erörtert.

Der H-Konzentrationsverlauf innerhalb der Multischichten während der Tiefenprofilierung ist in Abbildung 5.5 schematisch dargestellt. Zur Diskussion des

\title{
a) Gleichgewicht:
}

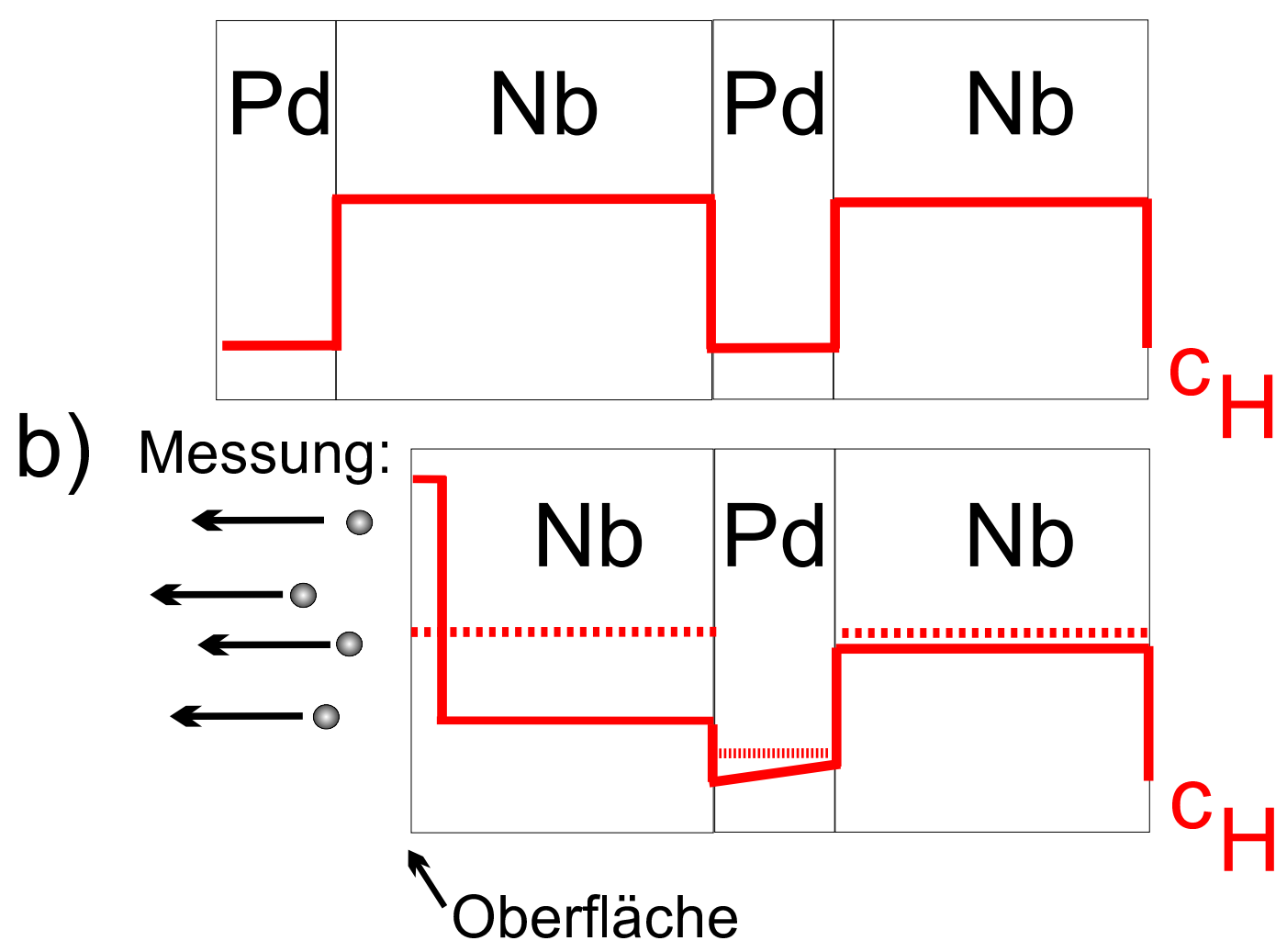

\begin{abstract}
Abbildung 5.5: Schematische Darstellung der H-Konzentration in einem Multischichtsystem a) vor und b) während der Tiefenprofilierung. Die Gleichgewichtsverteilung in a) verändert sich während der Messung zu einer Verteilung mit $H$-Konzentrationsgradienten b), da sich durch energetisch günstige Oberflächenplätze eine erhöhte H-Konzentration an der Oberfläche einstellt.

Einflusses der H-Diffusion auf die gemessenen H-Signale müssen bei der Tiefenprofilierung der Multischichten prinzipiell drei Diffusionsprozesse berücksichtigt werden. Wird eine Nb-Schicht gemessen, so sind dies die folgenden:
\end{abstract}

1. Die H-Diffusion aus dem Inneren der zu messenden Nb-Schicht an die Oberfläche, d.h. senkrecht zur Schichtebene. (Intraschichtdiffusion)

2. Die laterale H-Diffusion aus den Bereichen der Nb-Schicht, die nicht vom Sputterprozeß erfaßt wird. (Laterale Diffusion) 
3. Die H-Diffusion aus dem Pd/Nb-Schichtsystem, das hinter der zu messenden Nb-Schicht liegt. (Interschichtdiffusion)

\section{Intraschichtdiffusion}

Beim ersten Punkt handelt es sich zunächst um ein einfaches Diffusionsproblem in einer Einzelschicht. Durch die extrem schnelle H-Diffusion bei Raumtemperatur innerhalb einer Schicht kann der Konzentrationsverlauf in der vorderen Schicht als Kastenprofil genähert werden. Die Menge an H, die an der Oberfläche segregiert, wird dem hinteren Teil der Schicht entzogen. Durch das Absputtern sinkt die mittlere H-Konzentration, die Schicht wird sukzessive entleert. Ohne einen weiteren H-Antransport aus den Prozessen 2 und 3 erwartet man ein hohes HSignal, das nach der Entleerung der Schicht stark abfällt. Solche Signale zeigen die SIMS-Messungen (Abb. 5.2).

Bei der vollständigen Beladung sprechen sowohl die Höhe, als auch die Konstanz des Niveaus von $0,7 \mathrm{H} / \mathrm{Me}$ in den Nb-Schichten für eine Oberflächenkonzentration. Der vorzeitige Abfall am Ende der Nb-Schichten wird dann durch ein Versiegen des H-Stromes verursacht. In der mit 0,10 H/Nb beladenen Messung beobachtet man eine kurzzeitige Erhöhung der H-Konzentration an der der Oberfläche zugewandten Seite der Nb-Schichten und einen schnellen Abfall auf Konzentrationen, etwas oberhalb der nominellen Beladungskonzentration. Das Maximum der Konzentration erreicht jedoch nicht ganz die in der vollständig beladenen Messung detektierte maximale Konzentration, die dem Modell zu Folge der Oberflächenkonzentration entspricht. Im Fall der auf 0,04 H/Nb beladenen Messung reduziert sich der Anfangspeak nocheinmal deutlich. Durch die geringe Ausgangsmenge von Wasserstoff in den wenig beladenen Schichten reduziert sich der Konzentrationsgradient innerhalb der Schicht, so daß der H-Transport zur Oberfläche nicht mehr groß genug ist, um Konzentrationen um $1 \mathrm{H} / \mathrm{Me}$ zu erreichen.

\section{Laterale Diffusion}

Der zweite Diffusionsprozeß der lateralen Diffusion innerhalb der Nb-Schichten spielt bei den SIMS-Messungen eine vernachlässigbare Rolle. Dies konnte durch die Bestimmung der lateralen H-Konzentrationsverteilung im Sputterkrater beim Abtrag eines Pd/Nb-Schichtpaketes festgestellt werden. Dazu wurde mit der CsQuelle ein Krater gegraben und mit der Ga-Quelle die gesamte Kraterfläche analysiert. Abbildung 5.6 zeigt die laterale Verteilung der NbHCs-Signale in einem Sputterkrater von $(300 \times 300) \mu m^{2}$, aufgenommen mit einem Ga-Krater von ebenfalls $(300 \times 300) \mu m^{2}$.

Abbildung a) zeigt die lokale H-Verteilung an der oberflächenzugewandten Seite einer Nb-Schicht. Man erkennt eine gleichmäßige Konzentration in der Mitte des Kraters, der tiefsten Stelle des Sputterkraters. In Abbildung b) ist der hintere 


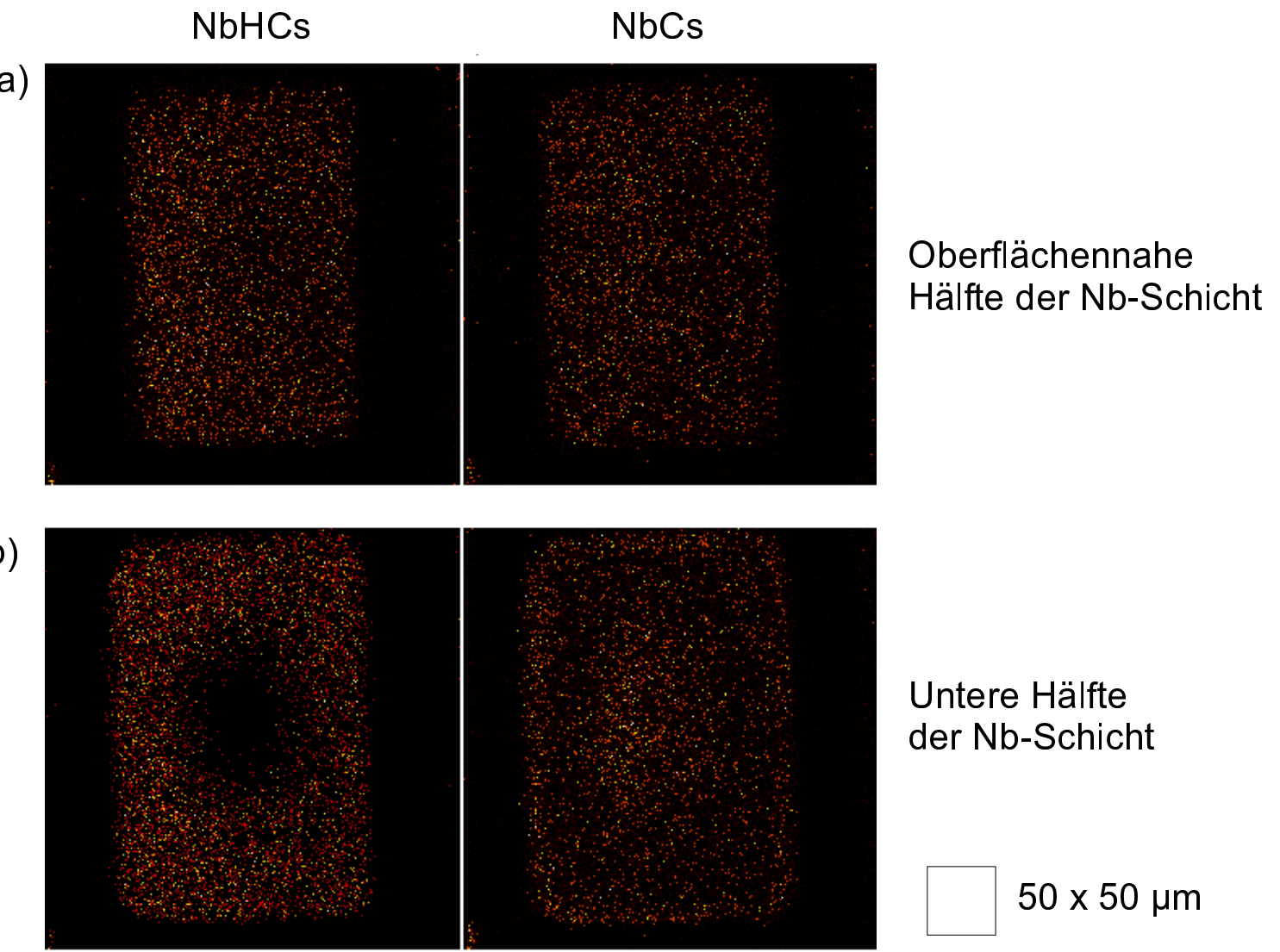

Abbildung 5.6: Laterale Verteilung der H-Konzentration in einer $15 \mathrm{~nm}$ dicken $\mathrm{Nb}$-Schicht während einer SIMS-Tiefenprofilierung in einem Cs-Sputterkrater von (300 x 300) $\mu^{2}$, analysiert auf einer Fläche von (300 x 300) $\mu^{2}$ mit Ga-Ionen. Abgebildet sind zwei aufeinanderfolgende Abschnitte des Tiefenprofils: a) Signale für $\mathrm{NbHCs}$ und $\mathrm{NbCs}$ in der oberflächennahen Hälfte und b) in der unteren Hälfte der Nb-Schicht.

Bereich derselben Nb-Schicht dargestellt. Man erkennt nun, daß in der Mitte des Kraters das H-Signal reduziert ist. In den Bereichen außerhalb, die auf einer anderen Höhe liegen und in denen das Hydrid noch nicht vollständig abgesputtert wurde, ist das H-Signal noch zu detektieren.

Bei Diffusionslängen von ca. $\frac{3 m m}{s}$ sollte ein Ausgleich der H-Konzentrationen in der Nb-Schicht innerhalb der Meßzeit, die im Bereich von mehreren Minuten pro Schicht liegt, stattfinden. Beobachtet wird jedoch eine Verarmung in der Mitte der Schicht. Daraus kann man schließen, daß ein lateraler Ausgleich der H-Konzentration nicht stattfindet. Wegen der durch den Sputterprozeß entstehenden Oberflächenplätze diffundiert der Wasserstoff stattdessen senkrecht zur Schicht an die Oberfläche. Die dafür zurückzulegenden Wege liegen im Schicht- 
dickenbereich, d.h. bei wenigen nm, und sind somit um Größenordnungen kürzer als die Wege in den lateralen Richtungen der Schicht.

Für eine quantitative Analyse der H-Konzentrationen am Rand des Kraters, etwa zur Untersuchung von Grenzflächeneffekten, waren die gefundenen Signalintensitäten zu gering.

\section{Interschichtdiffusion}

Der dritte Diffusionsprozeß betrifft nun den H-Transport von einer Nb-Schicht zur nächsten durch die dazwischenliegende Pd-Schicht. Das gering lösende $\mathrm{Pd}$ transportiert erst dann Wasserstoff von der hinteren Nb-Schicht in die vordere, wenn ein Konzentrationsgradient vorliegt, d.h. die Konzentration an der linken Grenzfläche geringer als die an der rechten Grenzfläche ist. Durch die geringe H-Löslichkeit des Pd im Verhältnis zum Nb (s. Tab. 2.8), fällt jedoch der Konzentrationsunterschied und somit die treibende Kraft der Diffusion entsprechend reduziert aus. Die gering lösende Schicht wirkt also als Diffusionsbarriere, deren Höhe mit steigendem Löslichkeitsunterschied zunimmt.

Das H-Diffusionproblem durch ein Multischichtsystem ist von Schmitz et al. [61] modelliert und unter gewissen Randbedingungen analytisch gelöst worden. Die beschriebene Verzögerung der effektiven H-Diffusion durch ein Multischichtsystem aus Metallen mit stark unterschiedlichen H-Löslichkeiten ist ein Resultat dieses Modells, das im folgenden kurz skizziert werden soll.

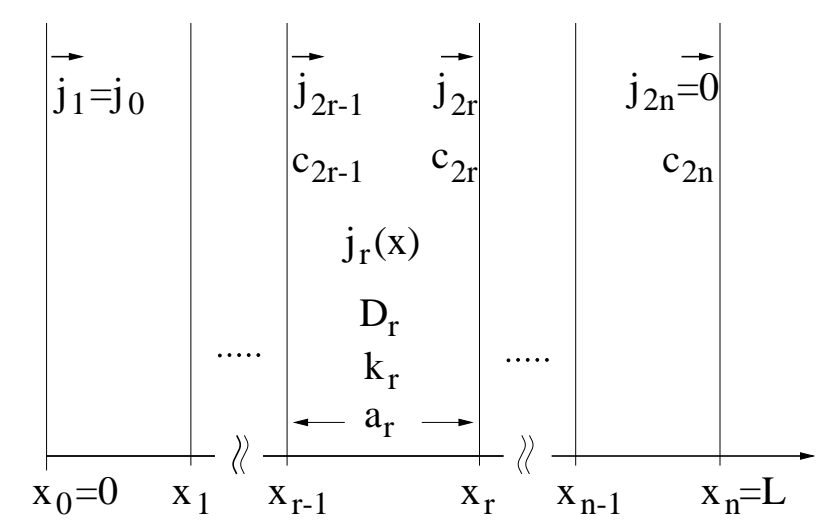

Abbildung 5.7: Schematischer Aufbau eines Multischichtsystems.

Modell zur H-Permeation durch metallische Multischichten In Anlehnung an die Behandlung der Diffusion durch Multischichten unter den Randbedingungen einer konstanten Konzentration, wie sie in [63] durchgeführt worden ist, wurde von Schmitz et al. [61] ein theoretisches Modell für die Diffusion entwickelt, für das man folgende Annahmen macht: 
1. Die Multischichten bestehen aus $n$ Lagen der Komponenten $r$. Die Dicke der Schicht $r$ sei $a_{r}$. Das Substrat habe die Dicke $s$.

2. Man betrachtet die Diffusion senkrecht zu den Multilagen durch eine Einheitsfläche.

3. Die Diffusionskoeffizienten $D_{r}$ für das diffundierende Medium $H$ in den Phasen $r$ seien konzentrationsunabhängig.

4. An den Grenzflächen $\left(x_{r}\right)$ wird für die Konzentrationen Henrysches Verhalten angenommen, was man durch eine Löslichkeitskonstante $k_{r}$ ausdrücken kann:

$$
\frac{c_{2 r}}{k_{2 r}}=\frac{c_{2 r}}{k_{2 r+1}}
$$

Dabei entspricht der Faktor $\frac{k_{r}}{k_{r+1}}$ dem in Gl.2.8 definiereten Löslichkeitsunterschied zwischen den Phasen $k$.

5. Die Randbedingungen sind entweder ein konstanter Strom oder eine konstante Konzentration an der Eintrittsseite

$$
j_{1}=\text { konst } \quad \text { bzw. } \quad c_{1}=\text { konst., }
$$

und ein verschwindener Strom bzw. eine verschwindene Konzentration an der Austrittsseite am Ende des Substrats

$$
j_{2 n}=0 \quad \text { bzw. } \quad c_{2 n}=0 .
$$

Die analytische Lösung der Diffusionsgleichungen für ein beliebiges Multischichtpaket gelingt unter diesen Annahmen. Die für eine Darstellung im Rahmen dieser Arbeit zu komplexe Lösung ist in [61] beschrieben. Hier soll zur Veranschaulichung die Lösung auf ein Pd/Nb-Multischichtsystem auf einem H-permeablem Pd-Substrat angewendet werden. Elektrochemische Permeationmessungen unter Konstantstrombedingungen an $\mathrm{Pd} / \mathrm{Nb}$-Systemen sind ebenfalls in [61] und [64] beschrieben. Weitere Messungen an $\mathrm{Pd} / \mathrm{Nb}-$ Systemen sind im Anhang aufgeführt. Die H-Permeation durch ein Pd/Nb-Multischichtsystem ist in Abb. 5.8 schematisch dargestellt.

Für den Fall eines Systems aus zwei Phasen $P d$ und $N b$ der Dicken $a_{P d}=$ $a_{N b}=a$ mit vielen und identischen Doppelschichten $(n \rightarrow \infty)$, einer Phase als Substrat, die auch als Multischichtphase auftritt $\left(k_{P d}=k_{S}\right)$ und eines hohen Löslichkeitsunterschieds zwischen den beiden auftretenden Phasen $\left(k_{N b} \gg\right.$ $k_{P d} ; k \rightarrow 0$ ) findet man für die Durchbruchzeit durch das Gesamtsystem, die die Verzögerung des Anstieges der H-Konzentration bei der Permeation durch ein Schichtsystem beschreibt,

$$
t_{L}=3 \cdot \frac{s^{2}}{6 \cdot D_{S}}+\frac{n^{2} \cdot a^{2}}{6 \cdot k \cdot D_{P d}} .
$$




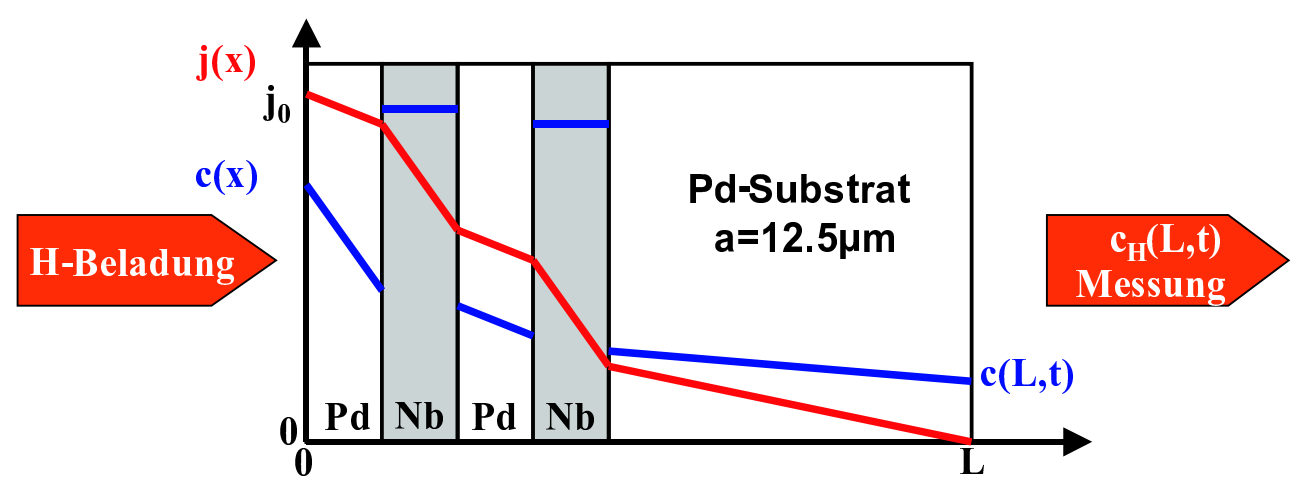

Abbildung 5.8: Schematische Darstellung der H-Permeation durch ein Pd/NbMultischichtsystem auf einem Pd-Substrat (Details im Anhang A).

Man erkennt, daß sich diese Lösung aus zwei Summanden zusammensetzt, von denen der erste der Durchbruchzeit für die Permeation durch das Substrat mal einem Faktor 3 ist

$$
t_{L}=3 \cdot t_{L}^{S u b}+t_{L}^{M L}
$$

mit

$$
t_{L}^{S u b}=\frac{s^{2}}{6 \cdot D_{S}}
$$

Diese stationäre Lösung der Diffusionsgleichung für eine einzelne Schicht ist z.B. in [65] beschrieben.

Den zweiten Summanden kann man als eine Durchbruchzeit für die Multischichten auffassen

$$
t_{L}^{M L}=\frac{n^{2} \cdot a^{2}}{6 \cdot k \cdot D_{P d}} .
$$

Es zeigt sich in der Modellösung, daß die Durchbruchzeit im Multischichtterm neben der Schichtgeometrie vor allem vom Löslichkeitsunterschied $k$ und vom Diffusionskoeffizienten in der gering lösenden Phase $D_{P d}$ abhängt. Für hohe Löslichkeitsunterschiede nimmt der zweite Summand große Werte an. Man erhält somit eine deutliche Verzögerung der Permeation in solchen Multischichtsystemen. Die Pd-Schichten lassen sich somit als Barriere betrachten.

Die Modellierung erlaubt es einem nun, experimentelle Daten für die Durchbruchzeiten mit den modellierten zu vergleichen. Dies ist im Anhang an Pd/NbSchichten mit 4, 8 und 16 Doppelschichten dargestellt. Man erhält aus dem Experiment eine Verzögerung der Permeation, die die modellierten Werte noch übersteigt. Die auftretenden Abweichungen wurden über Modifikationen des Modells auf mögliche Grenzflächeneffekte, wie sie in Abb. 2.1 skizziert sind, untersucht. Dies ist ebenfalls im Anhang beschrieben und wird in Abschnitt 5.7.2 diskutiert. Die Anwendung des Multischichtmodells auf mit dem SIMS untersuchte Schichtsysteme folgt später. 
Faßt man die Pd-Schichten als Barriere auf, ist es auch verständlich, daß der HKonzentrationsverlauf in den einzelnen Nb-Schichten ähnlich verläuft (vgl. Abb. 5.2). In der Messung des mit 0,10 H/Nb beladenen Schichpaketes der Abb. 5.2 erkennt man, daß die H-Konzentration in der ersten Nb-Schicht schnell abnimmt, obwohl in der zweiten Nb-Schicht noch Wasserstoff vorhanden ist, der aber erst beim Abtrag der zweiten Schicht detektiert wird. Der Transport durch das Pd ist somit deutlich langsamer als innerhalb der Nb-Schichten.

Die ansteigenden Konzentrationsprofile in den hinteren Teilen der Einzelschichten und die deutliche Abnahme der Gesamtkonzentration in der letzten Nb-Schicht (s. Tab. 5.1) deuten jedoch auf einen gewissen Transport aus den hinteren Schichten durch das Pd hin. Der Einfluß dieses Transportprozesses wird an den zeitabhängigen SIMS-Messungen am Schichtpaket mit 6 Doppellagen $\mathrm{Pd} / \mathrm{Nb}$ deutlich, die mit unterschiedlichen Abtragsraten durchgeführt wurden (Abb. 5.4). Wie in Abschnitt 5.2 beschrieben, findet man bei der schnelleren Messung, die mit der gleichen Abtragsrate wie die der Messsungen aus Abb. 5.2 durchgeführt wurde, wiederum hohe Anfangskonzentrationen innerhalb der Nb-Schichten, die noch innerhalb der Schicht abfallen. In der um einen Faktor von ca. 9 langsameren Messung ist das H-Signal innerhalb der Nb-Schichten nahezu konstant und in den ersten Schichten deutlich höher als in der schnellen Messung. Dagegen fällt das H-Signal ab der vierten Nb-Schicht unter das der schnellen Messung. Dies läßt sich verstehen, wenn man annimmt, daß der Wasserstoff in der langsamen Messung mehr Zeit hat, durch die Pd-Barrieren in die vorderen Nb-Schichten zu diffundieren, wo er das Meßsignal hoch hält. Erreicht man mit der Tiefenprofilierung die hinteren Schichten, so mißt man wegen der vorher stattgefundenen H-Diffusion in die vorderen Schichten hier eine reduzierte H-Konzentration.

Die maximale Höhe der detektierten H-Konzentration in der langsamen Messung an dem mit 0,10 H/Nb beladenen Schichtpaket liegt bei ca. 0,3 H/Me. Sie liegt unterhalb der Oberflächenkonzentration von $0,7 \mathrm{H} / \mathrm{Me}$, die in der vollständig beladenen Probe (Abb. 5.2c)) gemessen wurde. Die geringere Höhe kann durch den geringeren $\mathrm{H}$-Transport wegen geringerer Konzentrationsgradienten erklärt werden. Die Konzentration spiegelt somit die Höhe des Zuflusses wider. Durch die Abnahme des Zuflusses in den hinteren Schichten nimmt dann auch die gemessene Konzentration langsam ab.

Aus der langsamen und schnellen Messung läßt sich der effektive Diffusionskoeffizient für die Multischichtdiffusion abschätzen. Die beiden Messungen dienen dabei als Grenzfälle. Während in der schnellen Messung die Diffusion innerhalb einer Doppelschicht nicht ungehindert abläuft, ist in der langsamen Messung der Transport des Wasserstoff aus den hinteren Nb-Schichten in die vordere ausreichend, um in der gemessenen Nb-Schicht eine konstant hohe H-Konzentration aufrecht zu erhalten. Als Diffusionsweg kann man als obere Grenze die Gesamtdicke ansetzen. Als zugehörige Zeit kann man die Meßzeit ansetzen, die benötigt wird, um von der Pd/Nb-Grenzfläche in den Bereich der Nb-Schicht vorzudringen, in dem bei der schnellen Messung das hohe Anfangssignal abfällt. Dies ist 
bei ca. 1/5tel der Nb-Schichtdicke der Fall. Bei Annahme der einfachen parabolischen Beziehung zwischen der Diffusionslänge und Zeit (Gl. 5.3) erhält man als Abschätzung für den Diffusionskoeffizienten

$$
D=\frac{x^{2}}{t}
$$

Mit der Dicke von $150 \mathrm{~nm}$ und Meßzeiten von ca. $60 \mathrm{~s}$ für die schnelle und ca. $500 \mathrm{~s}$ für die langsame Messung erhält man die Abschätzung, daß der MultischichtDiffusionskoeffizient - indirekt gemessen mit dem SIMS - zwischen $4 \cdot 10^{-12}$ und $4 \cdot 10^{-13} \frac{\mathrm{cm}^{2}}{\mathrm{~s}}$ liegt.

Nach der exakten Lösung des Permeationsmodelles (s. oben und [61]) erwartet man für ein System aus mehreren Nb/Pd-Schichten mit $25 \mathrm{~nm}$ Doppelschichtdicke und den in den Tabellen 2.1 und 2.2 angegebenen H-Löslichkeiten und Diffusionskoeffizienten einen effektiven Diffusionskoeffizienten

$$
D_{\text {eff }}=\frac{a_{\text {gesamt }}^{2}}{2 \cdot t_{L}}=4 \cdot 10^{-11} \frac{\mathrm{cm}^{2}}{\mathrm{~s}} .
$$

Dabei wurde die mit dem Modell berechnete Gesamt-Durchbruchzeit auf die Gesamtdicke des Schichtpaketes bezogen. Als Randbedingungen sind dabei eine konstante H-Konzentration an der hinteren Nb-Oberfläche und eine verschwindene $\mathrm{H}-$ Konzentration an der vorderen Nb-Grenzfläche angenommen. Diese Randbedingungen sind sicher nicht direkt auf das Diffusionsproblem während der SIMSMessung übertragbar, jedoch ist als Abschätzung die verschwindene Konzentration mit dem Absputtern der NbH-Schicht an der Vorderseite und die konstante Konzentration mit der Konzentration in der hintersten Nb-Schicht vergleichbar. Die Konstanz der Konzentrationen ist sicher nicht gewährleistet, so daß der mit dem Modell berechnete Wert eine obere Grenze darstellt. Die Resultate zeigen somit im Rahmen der groben Näherung eine geringe Abweichung von ca. einer Größenordnung, was als weiterer Hinweis gewertet werden kann, daß das vorgeschlagene Modell der Oberflächensegregation die H-Transportvorgänge während der SIMS-Messung gut beschreibt.

Im nächsten Abschnitt werden nun die Ergebnisse der TAP-Analysen präsentiert. Das Interesse liegt hier insbesondere darin herauszufinden, inwieweit die tieferen Temperaturen bei der TAP-Analyse Messungen der H-Gleichgewichtsverteilungen erlauben.

\subsection{Messung der D-Verteilung bei tiefen Tem- peraturen (TAP-Analysen)}

Die Multischichtsysteme für die TAP-Messungen wurden wie in Kapitel 4 beschrieben auf W-Spitzen aufgebracht und mit Deuterium beladen. Aus den obigen Ergebnissen wird deutlich, daß die Bestimmung der D-Verteilung durch zerstörende, d.h. Oberflächen bildende Analysetechniken nur möglich ist, wenn es 
gelingt die Beweglichkeit des Deuteriums einzuschränken. Am FIM/TAP besteht die Möglichkeit zur Kühlung der Proben auf bis zu etwa $15 \mathrm{~K}$. Zudem ist die Tiefenauflösung des TAP deutlich besser als beim SIMS. TAP-Analysen bei tiefen Temperaturen von $60 \mathrm{~K}$ und $20 \mathrm{~K}$ werden im folgenden beschrieben. Gezeigt wird jeweils die in Abschnitt 3.1.2 erläuterte dreidimensionale Rekonstruktion der Meßergebnisse und ein Konzentrationsprofil, das aus einem zylindrischen Teilvolumen der Rekonstruktion gewonnen wurde. Der Analysezylinder ist in der Rekonstruktion eingezeichnet.

\subsection{1 $\mathrm{Pd} / \mathrm{Nb}-\mathrm{Multischichten}$}

Von den $\mathrm{Pd} / \mathrm{Nb}-\mathrm{Schich}$ tsystemen, die sich schon im unbeladenen Zustand sehr instabil zeigten, konnte im beladenen Zustand nur die letzte Nb-Schicht auf dem W-Substrat gemessen werden. Das Versagen der Schichten an der Pd/NbGrenzfläche wird auf die hohe Gitterfehlpassung von $\mathrm{Pd}$ und Nb zurückgeführt. Die D-Beladung führt zu einer Aufweitung des Nb-Gitters und somit zu zusätzlichen mechanischen Spannungen in den Schichtsystemen, denen offensichtlich die $\mathrm{Nb} / \mathrm{W}$-Grenzfläche mit einer sehr geringen Ausgangsgitterfehlpassung zunächst widersteht (s.Tab. 2.3). Die folgenden Messungen der Nb/W-Grenzfläche stammen deswegen alle von Proben, die ursprünglich als Pd/Nb-Multischichtsysteme präpariert waren. Sie wurden bei Raumtemperatur mit der Laserdeposition hergestellt.

\subsubsection{Nb/W-Grenzfläche}

Die Abbildungen 5.9 bis 5.14 zeigen Messungen solcher Nb-Schichten auf dem WSubstrat, die nach unterschiedlichen D-Beladungen und bei zwei Temperaturen (60 K und $20 \mathrm{~K}$ ) durchgeführt wurden.

\section{Messungen bei $60 \mathrm{~K}$}

Die nominell am stärksten beladene Schicht (13,5 mbar $\mathrm{D}_{2}$ über $\left.25 \mathrm{~h}\right)$ wurde bei $60 \mathrm{~K}$ gemessen. Die Rekonstruktion der Messung zeigt Abb. 5.9. Die detektierten Nb-Atome (blau) und D-Atome (rot) zeigen eine lagenförmige Verteilung. Das Volumen wurde mit einem mittleren Atomvolumen von $9^{3}$ rekonstruiert und beträgt 9,5 x 9,5 x 10,5 $\mathrm{nm}^{3}$. Abbildung 5.10 zeigt die im Zylinder durchgeführte Konzentrationsanalyse. Das Analysevolumen von $0,24 \mathrm{~nm}$ x $28,3 \mathrm{~nm}^{2}$ wurde schrittweise um 0,08 nm verschoben und umfaßt jeweils mehr als 250 Atome. Das Konzentrationsprofil zeigt über ca. $8 \mathrm{~nm}$ eine konstante D-Konzentration von $1 \mathrm{D} / \mathrm{Me}$, die anschliessend über $1 \mathrm{~nm}$ auf unter $0,1 \mathrm{D} / \mathrm{Me}$ abfällt. An das $\mathrm{Nb}$ schließt sich das $\mathrm{W}$ an. Bei der Erhöhung der Spannung auf den Feldverdampfungswert für $\mathrm{W}$ brach die Spitze an der Nb-W-Grenzfläche ab, so daß nur wenige $\mathrm{W}$-Atome detektiert werden konnten. Als waagerechte Linie eingezeichnet 


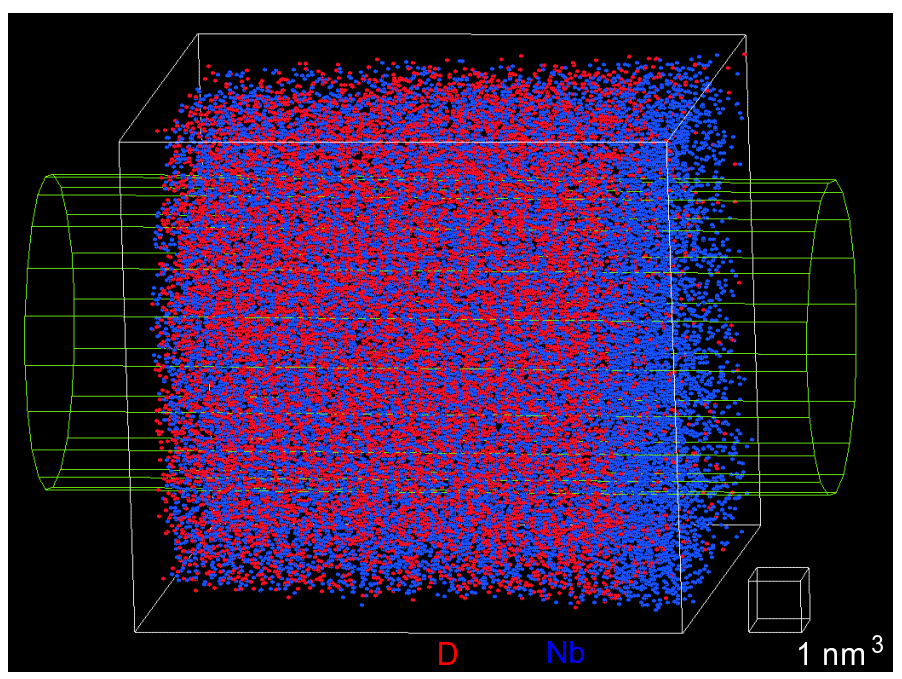

Abbildung 5.9: Rekonstruktion der TAP-Mesung einer Nb-Einzelschicht auf $W$, beladen mit 13,5 mbar $D_{2}$ und gemessen bei $60 \mathrm{~K}$. Man erkennt die ebenenförmige Anordnung der detektierten Nb-Atome (blau) und D-Atome (rot). Das rekonstruierte Volumen beträgt 9,5 x 9,5 x 10,5 $\mathrm{nm}^{3}$. Der eingezeichnete Zylinder markiert das Teilvolumen, in dem die Konzentrationsanalyse durchgeführt wurde (Abb. 5.10). Er hat einen Radius von $3 \mathrm{~nm}$.

ist zusätzlich die mittlere D-Konzentration im Inneren der Nb-Schicht mit 0,9 D/Me. Zur Berechnung wurde die D-Konzentration im Bereich der Schicht ausgewertet, in dem mehr als $90 \%$ der Metallatome als Nb gemessen wurden. Die Breite der Linie entspricht diesem Bereich der Nb-Schicht. Auch in den folgenden Messungen wird diese Definition für das Innere einer Schicht gewählt. Der Fehler in der Konzentrationsangabe liegt bei ca. 0,2 D/Me. Die Schwankungen in der D/Me-Kurve stammen von der lagenförmigen D-Verteilung im Zwischengitter der Nb-Schichten. Die Gitterebenen lassen sich in der Rekonstruktion (Abb. 5.9) erkennen. Das Analysevolumen mit atomarer Tiefe wurde gewählt, um Grenzflächen mit maximaler Auflösung darzustellen.

Abb. 5.11 zeigt eine weitere Nb-Schicht auf $\mathrm{W}$, die ebenfalls bei $60 \mathrm{~K}$ gemessen wurde ${ }^{2}$. Das Volumen wurde mit einem mittleren Atomvolumen von 14 ${ }^{3}$ rekonstruiert und beträgt $12 \times 12 \times 13 \mathrm{~nm}^{3}$. Die D-Beladung lag mit 2,5 mbar über $70 \mathrm{~h}$ niedriger, allerdings sollten nach den Druck-Konzentration-Isothermen für das Nb-D-System [31] die angewendeten Drücke beide um Größenordnungen über dem Druck zur Bildung der Nb-D- $\beta$-Phase liegen. Abbildung 5.12 zeigt die im Zylinder durchgeführte Konzentrationsanalyse. Das Analysevolumen von $0,24 \times 28,3 \mathrm{~nm}^{3}$ wurde schrittweise um $0,08 \mathrm{~nm}$ verschoben und umfaßt jeweils mehr als 160 Atome. Die D-Konzentration zeigt eine deutlich breitere DVerarmung, die auf die letzten $4 \mathrm{~nm}$ vor der Grenzfläche ausgedehnt ist.

\footnotetext{
${ }^{2}$ Auch hier brach die Spitze nach Messung der Nb-Schicht an der Nb/W-Grenzfläche ab.
} 


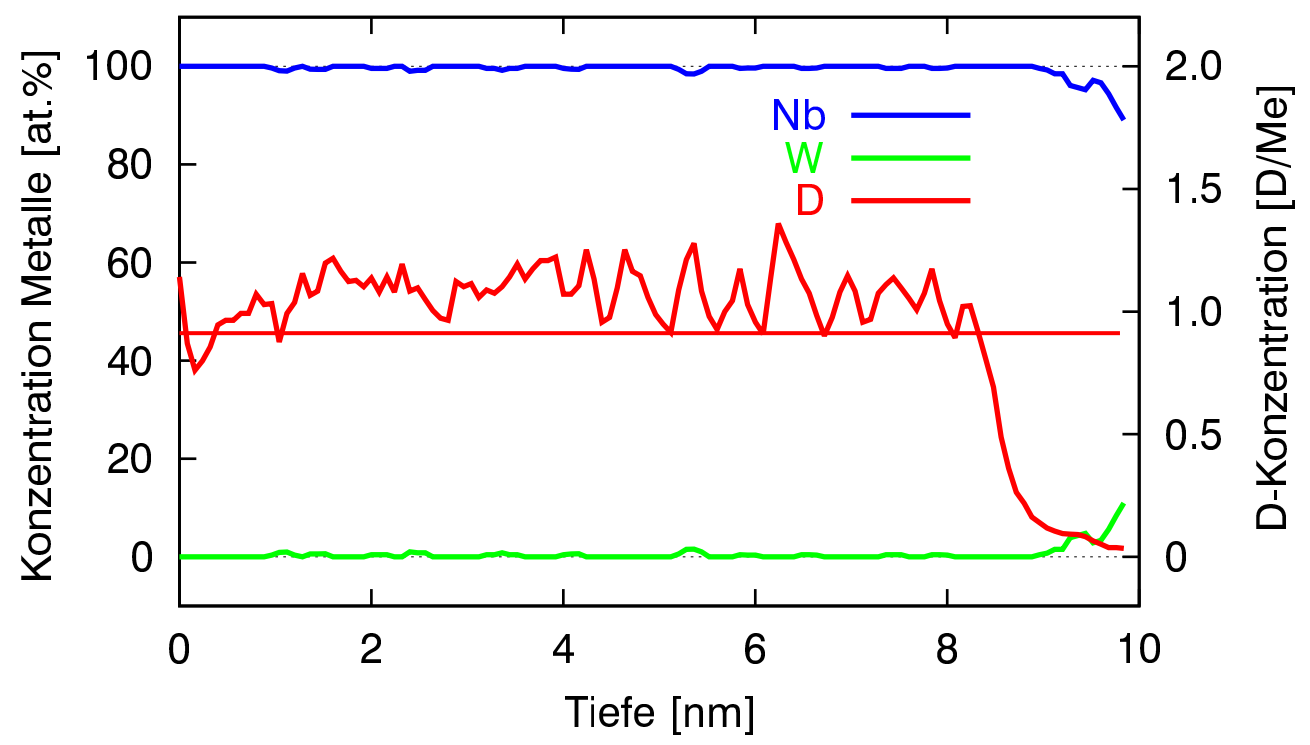

Abbildung 5.10: Konzentrationsprofil der Messung aus Abb. 5.9. Gemessen wurde von links nach rechts. An der linken Ordinate aufgetragen sind die Konzentrationen der Metalle, an der rechten das Verhältnis D/Metallatome (Analysevolumen: 0,24 $\mathrm{nm} x$ 28,3 $\mathrm{nm}^{2}$ ). Als waagerechte Linie eingezeichnet ist die mittlere D-Konzentration von 0,9 D/Me im Inneren der Nb-Schicht. Die D-Konzentration ist über fast die gesamte $\mathrm{Nb}$-Schicht konstant und fällt erst ca. $1 \mathrm{~nm}$ vor der $\mathrm{Nb} / \mathrm{W}$-Grenzfläche.

Die beiden Schichten zeigen also eine D-Verarmung vor der Grenzfläche, deren Ausdehnung anscheinend von der D-Beladungsstärke abhängt. Abweichend von der ersten Schicht liegt die D-Konzentration am Anfang der Schicht nur bei ca. $0,3 \mathrm{D} / \mathrm{Me}$ und steigt bis zu $0,8 \mathrm{D} / \mathrm{Me}$ in einer Tiefe von ca. $6 \mathrm{~nm}$. Über $1 \mathrm{~nm}$ fällt sie dann stark ab und verschwindet nahezu auf den letzten $4 \mathrm{~nm}$ vor der Grenzfläche. Die mittlere D-Konzentration im Inneren der Nb-Schicht beträgt 0,26 D/Me und liegt somit deutlich unterhalb der Konzentration in der ersten Schicht. Diese Abweichungen werden später diskutiert. 


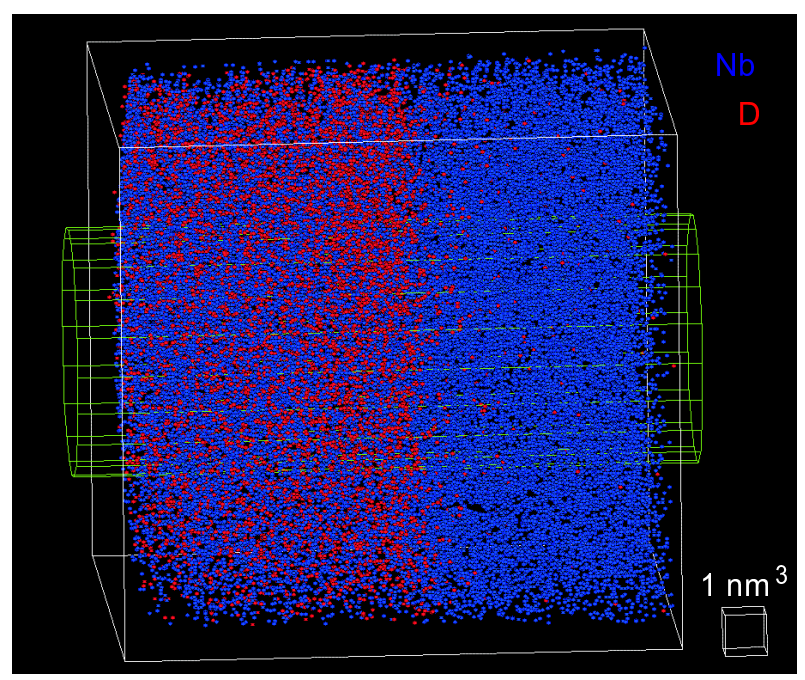

Abbildung 5.11: Rekonstruktion der TAP-Mesung einer Nb-Einzelschicht auf $W$, beladen mit 2,5 mbar $D_{2}$ und gemessen bei $60 \mathrm{~K}$. Die D-Atome (rot) werden in einem Teilbereich der Nb-Schicht (blau) weit weniger detektiert. Das rekonstruierte Volumen beträgt $12 \times 12 \times 13 \mathrm{~nm}^{3}$. Der eingezeichnete Zylinder markiert das Teilvolumen, in dem die Konzentrationsanalyse durchgeführt wurde (Abb. 5.12). Er hat einen Radius von $3 \mathrm{~nm}$.

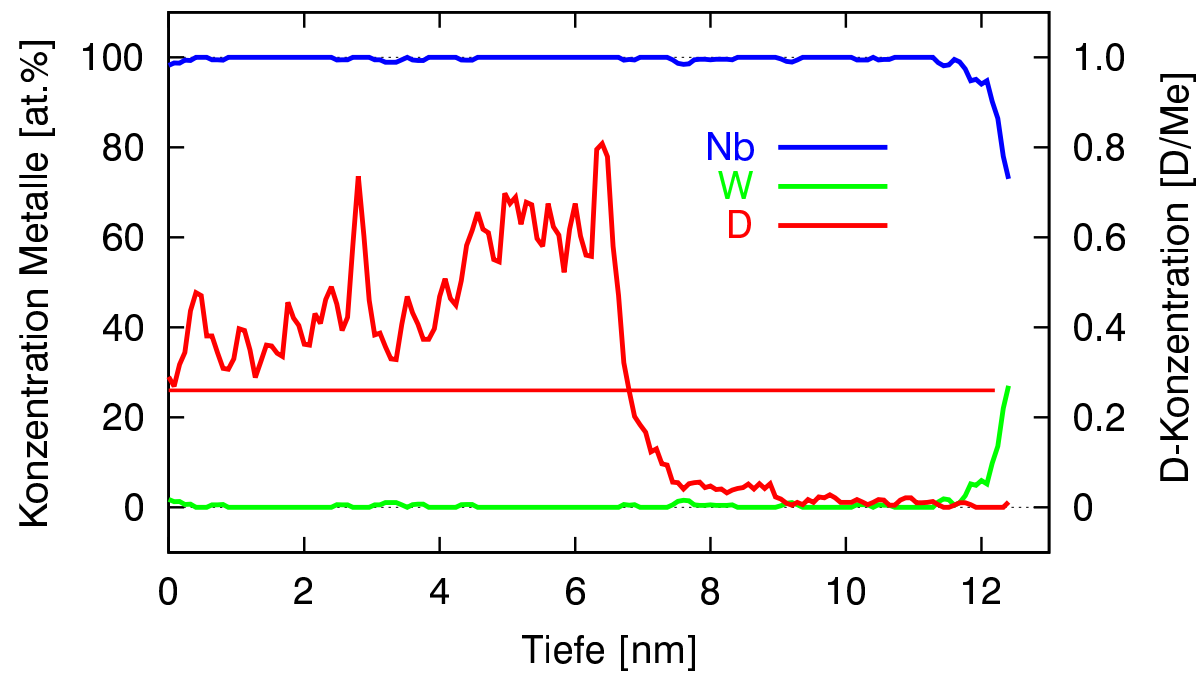

Abbildung 5.12: Konzentrationsprofil der Messung aus Abb. 5.11. Gemessen wurde von links nach rechts. An der linken Ordinate aufgetragen sind die Konzentrationen der Metalle, an der rechten das Verhältnis D/Metallatome (Analysevolumen: 0,24 $\mathrm{nm} x$ 28,3 $\mathrm{nm}^{2}$ ). Als waagerechte Linie eingezeichnet ist die mittlere D-Konzentration von 0,26 D/Me im Inneren der Nb-Schicht. Es wird eine ausgedehnte $D$-Verarmung von ca. $4 \mathrm{~nm}$ vor der $\mathrm{Nb} / W$-Grenzfläche gemessen. Ausserdem erkennt man einen Anstieg der D-Konzentration auf den ersten $6 \mathrm{~nm}$ der Nb-Schicht. 


\title{
Messungen bei $20 \mathrm{~K}$
}

Eine bei 1,9 mbar $\mathrm{D}_{2}$ über $23 \mathrm{~h}$ beladene Nb-Schicht auf $\mathrm{W}$ wurde bei $20 \mathrm{~K}$ gemessen. Die Rekonstruktion der Messung zeigt Abb. 5.13. Man erkennt neben den detektierten Nb-Atomen (blau) und D-Atomen (rot), die Gitterebenen des W-Substrates (grün). Das Volumen wurde wegen der geringen D-Konzentration mit dem Atomvolumen von $\mathrm{Nb}\left(18^{3}\right)$ rekonstruiert und beträgt $13 \times 13 \times 6$ $\mathrm{nm}^{3}$. Abbildung 5.14 zeigt die im Zylinder durchgeführte Konzentrationsanalyse. Das Analysevolumen von 0,21 nm x 28,3 $\mathrm{nm}^{2}$ wurde schrittweise um 0,07 nm verschoben und umfaßt jeweils mehr als 100 Atome.

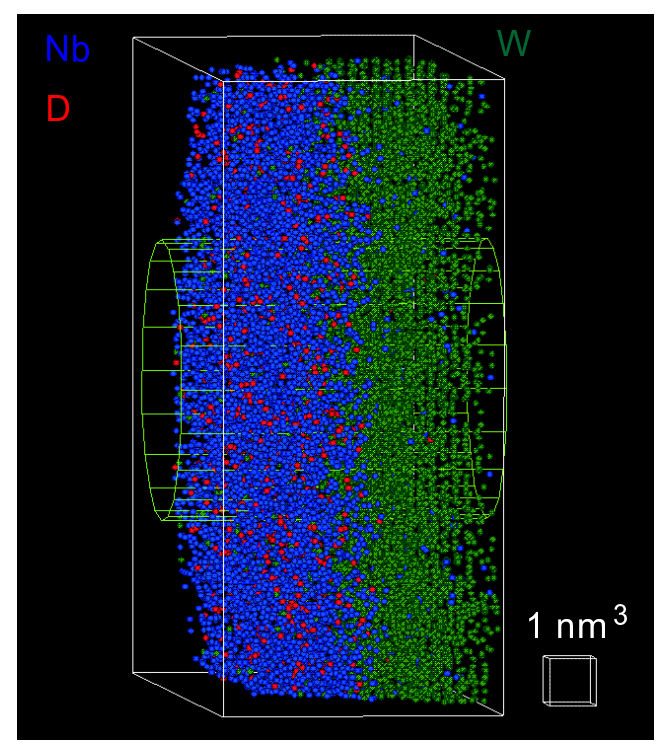

\begin{abstract}
Abbildung 5.13: Rekonstruktion der TAP-Mesung einer Nb-Einzelschicht auf $W$, beladen mit 1,9 mbar $D_{2}$ und gemessen bei $20 \mathrm{~K}$. Das rekonstruierte Volumen beträgt $13 \times 13 \times 6 \mathrm{~nm}^{3}$. Dargestellt ist die Nb-Schicht (blau) mit gelöstem Deuterium (rot) auf dem W-Substrat (grün). Zu erkennen sind die Gitterebenen des W. Der eingezeichnete Zylinder markiert das Teilvolumen, in dem die Konzentrationsanalyse durchgeführt wurde (Abb. 5.14). Er hat einen Radius von $3 \mathrm{~nm}$.
\end{abstract}

Vergleicht man diese Messung mit den Ergebnissen der Messungen bei $60 \mathrm{~K}$, so ergibt sich der folgende Unterschied. Die mittlere D-Konzentration liegt mit $0,14 \mathrm{D} / \mathrm{Me}$ deutlich unterhalb der oben gemessenen, reicht aber bis auf einige an die $\mathrm{Nb} / \mathrm{W}$-Grenzfläche heran. Dabei ist die Beladung bis auf die Dauer mit der von Abb. 5.11 vergleichbar. Der Beginn der Nb/W-Grenzfläche wird durch das Ende der waagerechten Linie gekennzeichet. Hier fällt die Nb-Konzentration unter 90 at.\% und das W-Signal steigt entsprechend an. Eine ausführliche Diskussion der Konzentrationsverläufe folgt in der Interpretation der Messungen in Abschnitt 5.7.2. Es zeigt sich somit eine homogenere D-Verteilung bei tieferen 


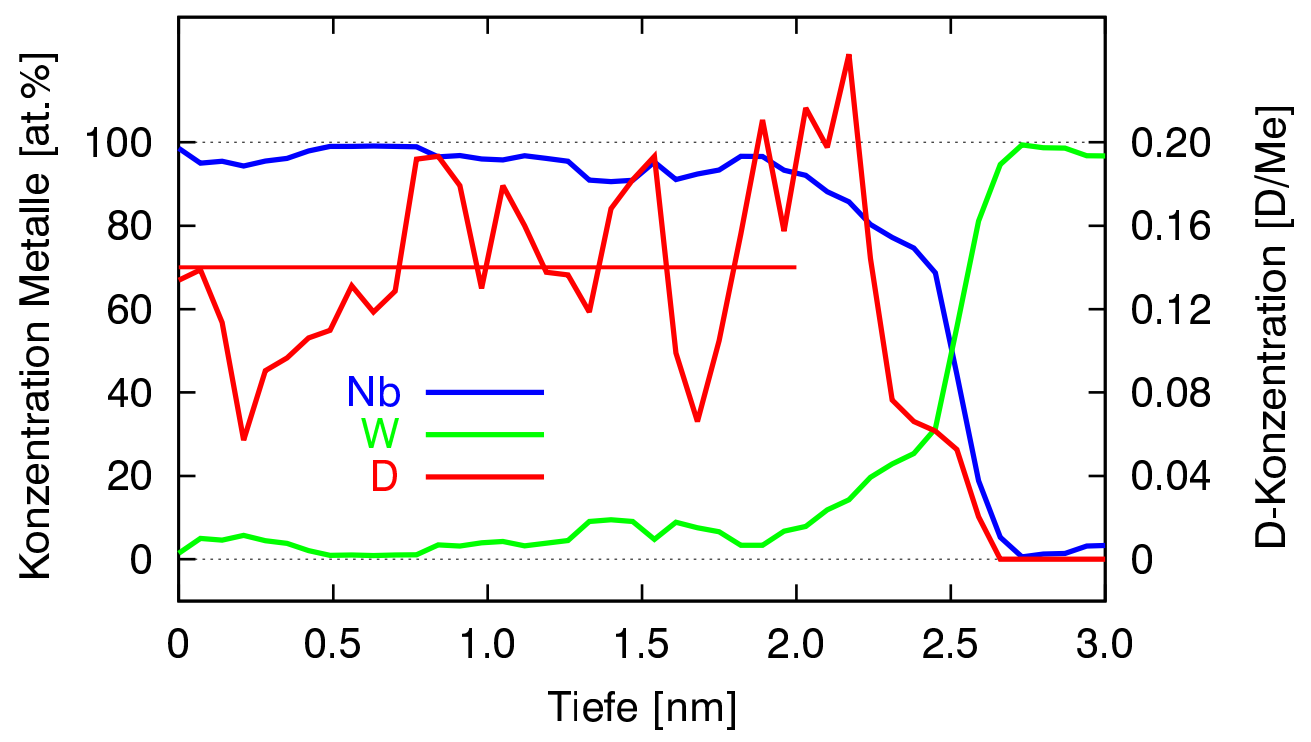

Abbildung 5.14: Konzentrationsprofil der Messung aus Abb. 5.13. Gemessen wurde von links nach rechts. An der linken Ordinate aufgetragen sind die Konzentrationen der Metalle, an der rechten das Verhältnis D/Metallatome (Analysevolumen: 0,21 $\mathrm{nm} *$ 28,3 $\left.\mathrm{nm}^{2}\right)$. Als waagerechte Linie eingezeichnet ist die mittlere D-Konzentration von 0,14 D/Me im Inneren der Nb-Schicht. Die DKonzentration wird bis zur $\mathrm{Nb} / W$-Grenzfläche mit ihrem mittleren Wert gemessen und fällt erst beim Anstieg der W-Konzentration.

Temperaturen, die bis an die Grenzfläche reicht. Dies wird auch deutlich, wenn man sich die nun folgenden Ergebnisse der Fe/V-Schichtsysteme ansieht.

\subsubsection{Fe/V-Schichtsysteme}

Auch die TAP-Analysen der D-beladenen Fe/V-Schichtsysteme wurden bei $60 \mathrm{~K}$ und $20 \mathrm{~K}$ durchgeführt. Die untersuchten Schichtsysteme wurden alle gesputtert, um eine bessere Vergleichbarkeit mit den von Hjörvarsson untersuchten Schichtpaketen zu erlangen.

\section{Messungen bei $60 \mathrm{~K}$}

Abbildung 5.15 zeigt die Rekonstruktion einer Messung eines beladenen Fe/VSchichtsystems bei $60 \mathrm{~K}$. Die Schichten wurden bei RT hergestellt und bei 2,5 mbar $\mathrm{D}_{2}$ für 90 min beladen. Das Volumen wurde mit einem mittleren Atomvolumen von $13,2^{3}$ rekonstruiert und beträgt $6 \times 6 \times 35 \mathrm{~nm}^{3}$. Man erkennt zwei $\mathrm{V} /$ Fe-Doppelschichten (blau/grau) mit einer Pd-Deckschicht (gelb). Deuterium (rot) ist fast ausschließlich in V gelöst, wie es der große Löslichkeitsunterschied im Vergleich zum Fe und auch zum Pd erwarten läßt (vgl. Tab. 2.4). 


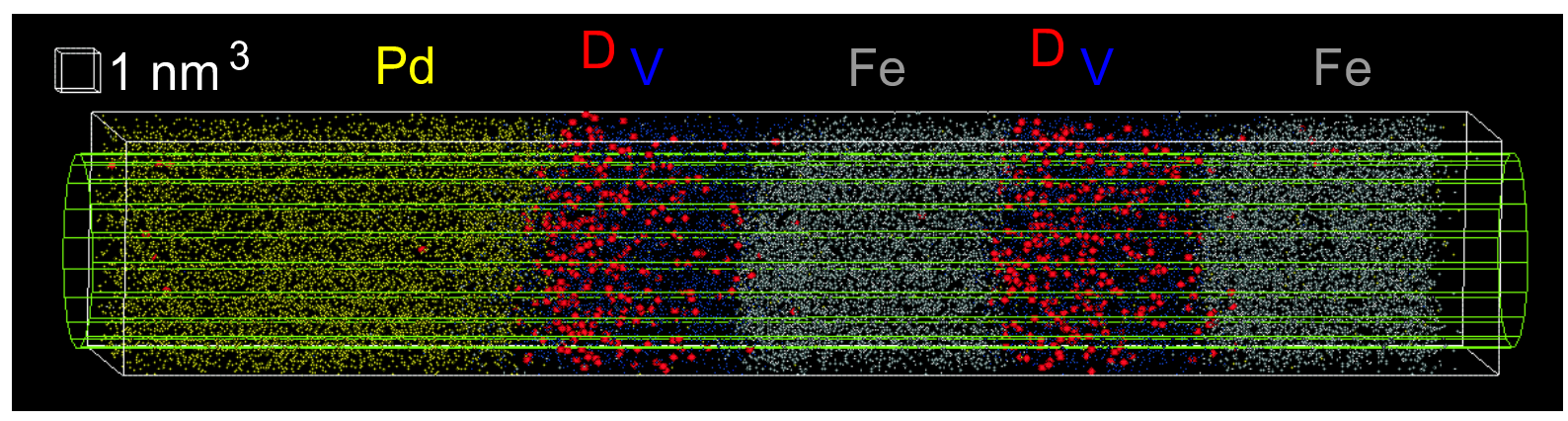

Abbildung 5.15: Rekonstruktion der TAP-Mesung von 2 Doppelschichten $V / F e$ auf $W$ mit Pd-Deckschicht, beladen mit 2,5 mbar $D_{2}$ und gemessen bei $60 \mathrm{~K}$. Dargestellt sind folgende Atomsorten: D (rot), V (blau), Fe(grau) und Pd (gelb). Das rekonstruierte Volumen beträgt $6 \times 6 \times 35 \mathrm{~nm}^{3}$. Der eingezeichnete Zylinder markiert das Teilvolumen, in dem die Konzentrationsanalyse durchgeführt wurde (Abb. 5.16). Er hat einen Radius von 2,5 nm. Man erkennt, daß das Deuterium fast ausschließlich in den V-Schichten gelöst ist.

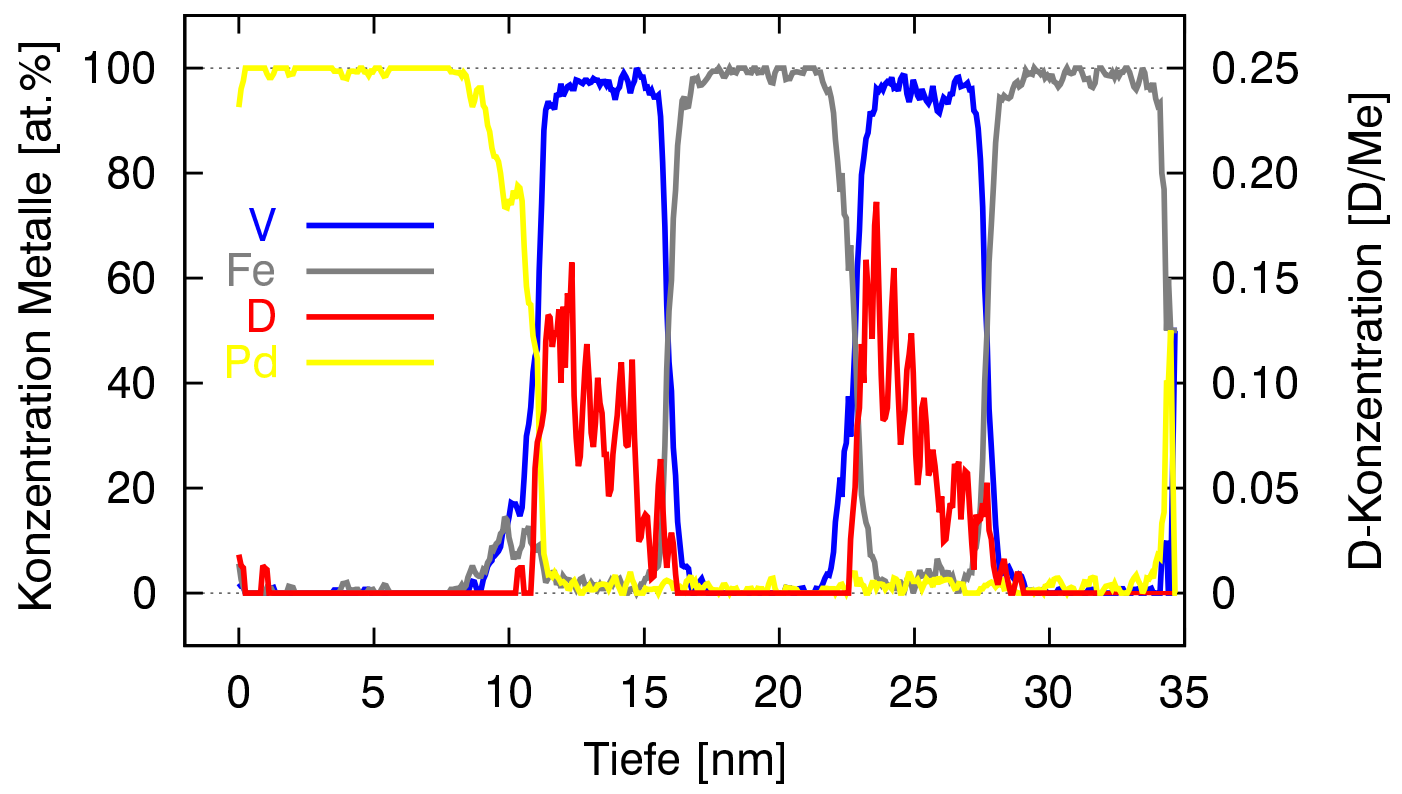

Abbildung 5.16: Konzentrationsprofil des Fe/V-Schichtpakets bei $60 \mathrm{~K}$ (vgl. Abb. 5.15). Gemessen wurde von links nach rechts. An der linken Ordinate aufgetragen sind die Konzentrationen der Metalle, an der rechten das Verhältnis D/Metallatome (Analysevolumen: 0,24 $\mathrm{nm} x$ 19,6 $\mathrm{nm}^{2}$ ). In beiden V-Schichten zeigt die D-Konzentration eine vergleichbare Höhe und einen ähnlichen Verlauf innerhalb der Schicht. Das D-Profil steigt in beiden Fällen innerhalb der VSchichten zur Meßseite an. 
Der im eingezeichneten Zylinder bestimmte Konzentrationsverlauf ist in Abb. 5.16 dargestellt. Das Analysevolumen (0,24 nm x 19,6 nm²) wurde schrittweise um 0,08 nm verschoben und umfaßt jeweils mehr als 100 Atome. Der Konzentrationsverlauf bestätigt, daß das Deuterium fast ausschließlich in den V-Schichten gelöst ist. Die D-Verteilung in beiden V-Schichten zeigt einen hohen Anfangswert (bei geringer Tiefe in Abb. 5.16) von ca. 0,15 D/Me, der jeweils zur hinteren Grenzfläche auf Werte unterhalb von 0,05 D/Me abfällt.

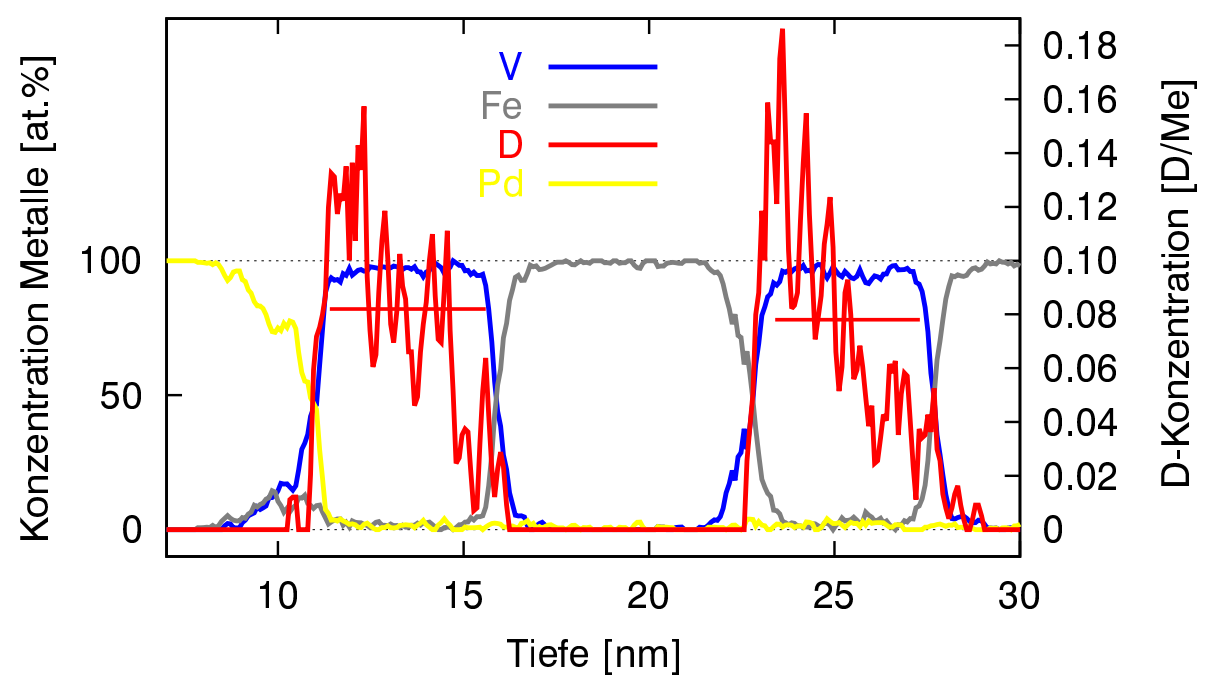

\begin{abstract}
Abbildung 5.17: Ausschnitt des Konzentrationsprofils des Fe/V-Schichtpakets bei $60 \mathrm{~K}$ (vgl. Abb. 5.16). Die D-Konzentration ist zum besseren Vergleich mit den Metallkonzentrationen hochskaliert. Als Balken eingezeichnet sind die mittleren D-Konzentrationen von 0,078 und 0,082 D/Me im Inneren der V-Schichten, d.h. in Bereichen, in denen die $V$-Konzentration $>0,9 \mathrm{~V} / \mathrm{Me}$ ist. Die DKonzentration nimmt an den Grenzfächen simultan mit der V-Konzentration $a b$.
\end{abstract}

In Abbildung 5.17 ist die D-Konzentration zum besseren Vergleich mit den Metallkonzentrationen hochskaliert. Der statistische Fehler der Konzentrationsbestimmung (s. Gl. 3.4) liegt etwas unterhalb der Schwankungen im Konzentrationsprofil. Eingezeichnet sind als waagerechte Linien die mittleren D-Konzentrationen von 0,078 und 0,082 D/Me im Inneren der V-Schichten. Es ist deutlich zu erkennen, daß die Abnahme des D-Signals an den Grenzflächen simultan mit der Abnahme der V-Konzentration verläuft. An der hinteren Grenzfläche ist lediglich die Höhe des Signals reduziert.

\title{
Messungen bei $20 \mathrm{~K}$
}

Abbildung 5.18 zeigt eine Messung, die bei $20 \mathrm{~K}$ an einem Doppelschichtsystem durchgeführt wurde, das bei Temperaturen von über $600^{\circ} \mathrm{C}$ hergestellt und mit 
6 mbar $\mathrm{D}_{2}$ für 20 min beladen wurde. Das Volumen wurde mit einem mittleren Atomvolumen von $13,2^{3}$ rekonstruiert und beträgt $6 \times 6 \times 35 \mathrm{~nm}^{3}$.

Die D-Konzentration (5.19) wurde im eingezeichneten Zylinder bestimmt. Das Analysevolumen von $0,24 \mathrm{~nm} \times 19,6 \mathrm{~nm}^{2}$ wurde schrittweise um 0,08 nm verschoben und umfaßt jeweils mehr als 100 Atome. Das D-Profil zeigt einen von den Messungen bei $60 \mathrm{~K}$ abweichenden Verlauf. Innerhalb der V-Schicht erkennt man eine konstante D-Konzentration, die sich bis zur V/Fe-Grenzfläche erstreckt. In Abbildung 5.20 ist die D-Konzentration zum besseren Vergleich mit den Metallkonzentrationen hochskaliert. Eingezeichnet ist als waagerechte Linie die mittlere D-Konzentration von 0,09 D/Me im Inneren der V-Schicht. Die Abnahme des D-Signals an der $\mathrm{V} / \mathrm{Fe}-$ Grenzfläche verläuft wiederum simultan mit der Abnahme der V-Konzentration. An der Pd/V-Grenzfläche hingegen erkennt man einen im Verhältnis zum V-Signal um ca. 2 nm verzögerten Anstieg des D-Signals. Die Diskussion dieser Abweichungen und eine genauere Analyse der Grenzflächenkonzentrationen folgen im Rahmen der Interpretation der Ergebnisse in Abschnitt 5.7 .2

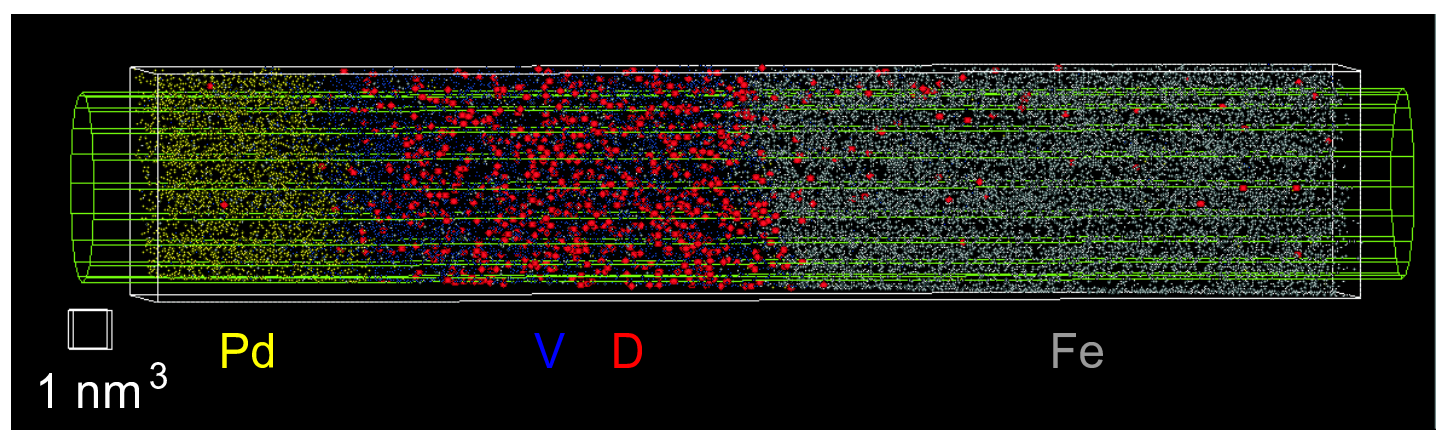

Abbildung 5.18: Rekonstruktion der TAP-Mesung einer Fe/V-Doppelschicht auf $W$ mit Pd-Deckschicht, beladen mit 6 mbar $D_{2}$ und gemessen bei $20 \mathrm{~K}$. Dargestellt sind folgende Atomsorten: D (rot), V (blau), Fe(grau) und Pd (gelb). Das rekonstruierte Volumen beträgt $6 \times 6 \times 35 \mathrm{~nm}^{3}$. Der eingezeichnete Zylinder markiert das Teilvolumen, in dem die Konzentrationsanalyse durchgeführt wurde (Abb. 5.16). Er hat einen Radius von 2,5 nm. Man erkennt, daß das Deuterium fast ausschließlich in der V-Schicht gelöst ist.

Die Ergebnisse der TAP-Messungen werden nocheinmal zusammengefaßt.

- Es ist gelungen stabile, metallische Multischichtsysteme für die analytische Feldionenmikroskopie zu präparieren, die im Fall des Nb/W-Systems mit Grenzflächenschärfen unter $1 \mathrm{~nm}$ gemessen werden können. Bei den anderen Systemen liegt die Grenzflächenschärfe je nach Depositionsmethode und Herstellungstemperatur bei 1-3 $\mathrm{nm}$.

- Über eine Gasphasenbeladung konnten die Systeme mit Deuterium beladen werden. Die D-Verteilung konnte mit dem TAP bestimmt werden. 


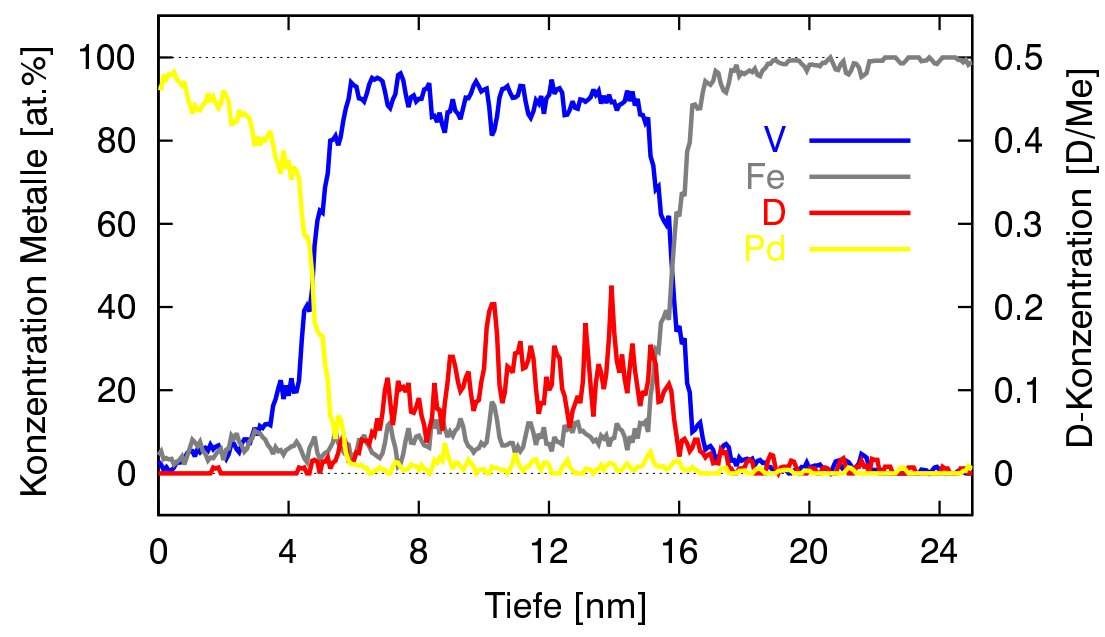

Abbildung 5.19: Konzentrationsprofil der Messung der Fe/V-Doppelschicht auf $W$ mit Pd-Deckschicht bei $20 \mathrm{~K}$ (vgl. Abb. 5.18). Gemessen wurde von links nach rechts. An der linken Ordinate aufgetragen sind die Konzentrationen der Metalle, an der rechten das Verhältnis D/Metallatome (Analysevolumen: 0,24 $\mathrm{nm} x$ 19,6 $\left.\mathrm{nm}^{2}\right)$. Innerhalb der $V$-Schicht wird eine konstante D-Konzentration bis an die $V / F e-G r e n z f l a ̈ c h e ~ b e o b a c h t e t$. An der Pd/V-Grenzfläche beobachtet man einen im Vergleich zum V-Signal verzögerten Anstieg der D-Konzentration.

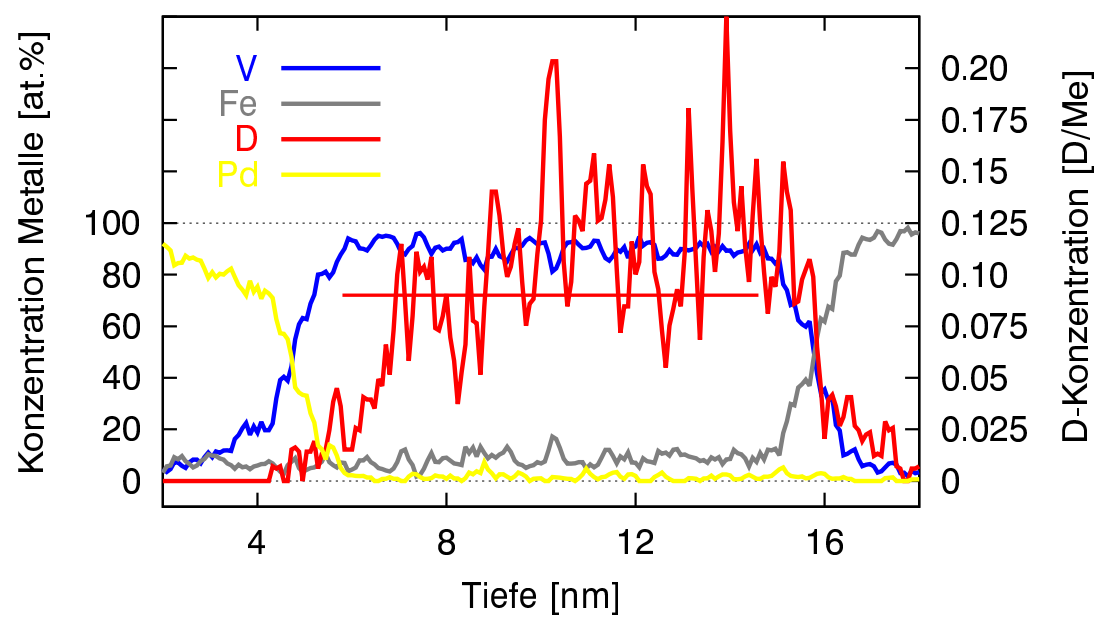

Abbildung 5.20: Ausschnitt des Konzentrationsprofils der Fe/V-Doppelschicht mit Pd-Deckschicht bei $20 \mathrm{~K}$ (vgl. Abb. 5.19). Die D-Konzentration ist zum besseren Vergleich mit den Metallkonzentrationen hochskaliert. Als Balken eingezeichnet ist die mittlere D-Konzentration im $V$ in dem Bereich, in dem die $V$-Konzentration > 0,9 V/Me ist. Die D-Konzentration nimmt an der $\mathrm{V} / \mathrm{Fe}$ Grenzfläche simultan mit der $V$-Konzentration ab. An der Pd/V-Grenzfläche beobachtet man einen im Vergleich zum $V$-Signal um ca. 2 nm verzögerten Anstieg der D-Konzentration. 
- Für Temperaturen oberhalb von $20 \mathrm{~K}$ wurden sowohl im Nb als auch im V D-Profile gefunden, die zur Meßrichtung hin ansteigen.

- In den Nb-Schichten haben die ansteigenden Profile der Messungen bei $60 \mathrm{~K}$ Stufencharakter, d.h. man findet anfangs hohe D-Signale und einen schnellen Abfall auf kleine D-Konzentrationen mit D-Verarmungsbereichen von bis zu $4 \mathrm{~nm}$.

- Bei Temperaturen von $20 \mathrm{~K}$ mißt man D-Profile, die innerhalb der Nb- und V-Schichten einen konstanten Verlauf zeigen.

- An der Nb/W- und V/Fe-Grenzfläche wird bei $20 \mathrm{~K}$ ein Abfall der DKonzentration simultan mit der Abnahme des Nb- bzw. V-Signals gemessen.

- An der bei über $600^{\circ} \mathrm{C}$ hergestellten $\mathrm{Pd} / \mathrm{V}$-Grenzfläche wird bei $20 \mathrm{~K}$ ein im Verhältnis zum V-Signal um ca. 2 nm verzögerter Anstieg des D-Signals gemessen.

\subsection{Diskussion der Ergebnisse der Messungen bei $60 \mathrm{~K}$}

Die Untersuchungen zur lokalen H-Verteilung in metallischen Multischichten bei $300 \mathrm{~K}$ (Abschnitt 5.2) haben verdeutlicht, daß Wasserstoff als kleinstes Element mit seiner hohen Beweglichkeit auf den Zwischengitterplätzen der Metalle sehr empfindlich auf Veränderungen der Proben reagiert. So beobachtet man im SIMS bei Raumtemperatur abhängig von der Meßzeit unterschiedliche HKonzentrationsprofile. Wie in Abschnitt 5.3 gezeigt, können diese auf die Segregation von Wasserstoff an den Probenoberflächen während der Messungen zurückgeführt werden. Am TAP dagegen besteht die Möglichkeit, die Proben bis auf $15 \mathrm{~K}$ zu kühlen. Messungen bei 20 und $60 \mathrm{~K}$ wurden im vorigen Abschnitt vorgestellt. Die folgende Diskussion beschäftigt sich nun mit der Frage, ob die Diffusion bei den gewählten Temperaturen unterdrückt ist.

\subsubsection{Einfluß der D-Diffusion bei $60 \mathrm{~K}$}

Auch in den dargestellten TAP-Analysen an Nb/W- und Fe/V-Schichtsystemen bei $60 \mathrm{~K}$ finden sich Hinweise auf Diffusionsprozesse des Deuteriums während der Messsung. In den Nb/W-Systemen erkennt man, ähnlich wie beim SIMS, bei kleinen Meßzeiten hohe D-Konzentrationen, die später abfallen. Der Abfall des D-Signals in den Nb-Schichten hat jeweils Stufencharakter, d.h. die Konzentration sinkt von hohen Werten sehr schnell innerhalb von $1 \mathrm{~nm}$ auf niedrige Konzentrationen ab. 
Die inhomogene D-Verteilung in der geringer beladenen Schicht (Abb. 5.12) ist der stärkste Hinweis, daß auch bei $60 \mathrm{~K}$ im TAP ein Diffusionsprozeß beobachtet wird. Während in der stärker beladenen Schicht (Abb. 5.10) die Abnahme der D-Konzentration erst kurz vor Erreichen der Grenzfläche einsetzt (und deshalb mit einem Grenzflächeneffekt fehlinterpretiert werden könnte), werden in der geringer beladenen Schicht die letzten $4 \mathrm{~nm}$ der Nb-Schicht mit sehr geringen D-Konzentrationen gemessen. Diese große Ausdehnung schließt einen Grenzflächeneffekt als Ursache aus.

Geht man wie bei den SIMS-Ergebnissen von einem Intraschicht-Diffusionprozeß aus, so kann die Verarmung am Ende der Schicht dadurch erklärt werden, daß es wieder durch die Diffusion des Wasserstoffs zur jeweils freigelegten Oberfläche zu einer sukzessiven Entleerung der Schicht beim Abtrag der Probe kommt. Denn auch die TAP-Analyse bietet dem Wasserstoff nach dem Abtrag der obersten Atomlage jeweils wieder neue Oberflächenplätze. Die Ausdehnung der Verarmungszone muß dann von der Ausgangskonzentration in der Schicht abhängen. So wird für stärker beladene Schicht eine deutlich geringere Ausdehnung gemessen als in der mit niedriger Konzentration.

Dem Segregationsmodell zufolge würde gleichzeitig die Konzentration am Anfang der Schicht erhöht detektiert werden. In der einen Schicht werden nahezu $1 \mathrm{D} / \mathrm{Nb}$ über fast die gesamte Schicht gemessen. In diesem Fall ist die mittlere Konzentration mit 0,9 D/Me auch nur unwesentlich kleiner. Der analysierte Teil der Nb-Schicht befindet sich der hohen mittleren Konzentration zufolge somit in der $\beta$-Phase des Nb-D-Systems.

Diese Messung zeigt zudem, daß das Deuterium mit vergleichbarer Detektionswahrscheinlichkeit wie die Metallatome nachgewiesen wird. Konzentrationen von maximal $1 \mathrm{D} / \mathrm{Nb}$ entsprechen den erwarteten Werten.

In der geringer beladenen Schicht (Abb. 5.12) findet man zunächst mittlere Konzentrationswerte von 0,3 D/Me, die zur Mitte der Schicht auf maximale Konzentrationen von ca. 0,8 D/Me ansteigen. Daß das Maximum der Konzentration nicht am Anfang der Schicht gemessen wird, kann mit den Vorstellungen über die Oberflächensegregation nur in Einklang gebracht werden, wenn die DKonzentration in der Nb-Schicht schon vor der Messung keinen konstanten Wert über die gesamte Breite der Schicht besessen hat. Die Ursache hierfür könnte die Existenz einer Ausscheidung der Hydridphase sein, die sich nicht über das gesamte analysierte Volumen von $12 \times 12 \times 13 \mathrm{~nm}^{3}$ erstreckt. Die geringe mittlere D-Konzentration von 0,26 D/Me zeigt deutlich, daß die Probe nicht vollständig hydriert ist und liegt sehr deutlich unterhalb des Konzentrationswertes von 0,9 $\mathrm{D} / \mathrm{Me}$, der in der ersten Schicht gefunden wird. Demzufolge muß sich das System noch im Zweiphasengebiet des Nb-D-Systems befinden. Die Konzentrationswerte von ca. 0,3 D/Me am Anfang der Schicht würden dann den D-Transport von der Ausscheidung an die Oberfläche widerspiegeln. Die höhere Konzentration in der Mitte der Schicht von ca. 0,7 D/Me gäben dann die Restkonzentration der Ausscheidung an. 
Der große Unterschied der mittleren D-Konzentrationen in den beiden Schichten (Abbn. 5.10 und 5.12) läßt sich entweder damit erklären, daß im Falle der ersten Schicht die Ausscheidung lateral vollständig im analysierten Volumen lag, während bei der zweiten Schicht das Zentrum der Aussscheidung nicht im Zentrum des analysierten Volumen gelegen hat. Andererseits kann aber auch der höhere Beladungsdruck (13,5 mbar im Vergleich zu 2,5 mbar bei der Beladung der zweiten Schicht) zu einer deutlich höheren Konzentration in der ersten NbSchicht geführt haben, so daß die Schicht vollständig hydriert, während die zweite Schicht nur einen kleinen Volumenbruchteil an Hydrid besitzt. Da es sich um Dünnschichtsysteme handelt, geben die Druck-Konzentration-Isothermen für das massive Nb-D-System [31] darüber keinen Aufschluß. Der Einfluß der Schichtdicke auf die Phasenbildung soll in Abschnitt 5.7.2 näher diskutiert werden.

Im Fe/V-Schichtsystem, das bei $60 \mathrm{~K}$ gemessen wurde (Abb. 5.17), verläuft der Anstieg der D-Konzentration zur Meßseite hin annähernd linear. Ein stufenförmiges D-Konzentrationsprofil wie in den Nb-Schichten wird nicht beobachtet. Das läßt bei der gewählten Temperatur von $60 \mathrm{~K}$ auf eine langsamere D-Diffusion im V als im Nb schließen. Dies steht im Widersruch zu experimentellen Daten bei höheren Temperaturen (s. Abb. 5.21), die das Gegenteil finden. Das lineare D-Profil im V zeigt, daß Deuterium im Verlauf der Messung nicht schnell genug zur Oberfläche diffundieren kann, um den hinteren Teil der Schicht vollständig zu entleeren. Dies läßt sich für eine grobe Abschätzung des Diffusionskoeffizienten auswerten. Die obere Grenze der D-Diffusion ist somit ein Diffundieren durch die gesamte Schichtdicke von $5 \mathrm{~nm}$ in der Meßzeit, die bei ca. $300 \mathrm{~s}$ lag. Mit Gl. 5.10 folgt

$$
D_{D, V}(60 K)<8 \cdot 10^{-16} \frac{\mathrm{cm}^{2}}{\mathrm{~s}} \text {. }
$$

Die D-Diffusion in $\mathrm{Nb}$ ist den TAP-Messungen zufolge schneller, da sich in der Nb-Schicht ein stufenförmiges Profil ausbildet. Das Deuterium ist somit schnell genug, um in der Meßzeit die gesamte Breite der Nb-Schicht zu durchqueren. Hier gelingt somit nur eine untere Abschätzung. Bei einer Schichtdicke von $12 \mathrm{~nm}$ und einer Meßzeit von ca. 2000 s ergibt sich

$$
D_{D, N b}(60 K)>7 \cdot 10^{-16} \frac{\mathrm{cm}^{2}}{\mathrm{~s}} \text {. }
$$

Abbildung 5.21 zeigt die Diffusionskoeffizienten von Deuterium in V und Nb. Die durchgezogenen Linien im Bereich von $200 \mathrm{~K}$ bis $300 \mathrm{~K}$ sind der Literatur entnommen [66]. Als gestrichelte Linien eingezeichnet sind die einfachen linearen Extrapolationen des Hochtemperaturbereichs über viele Größenordnungen. Messungen und mögliche Diffusionsmechanismen bei tiefen Temperaturen sind in [33] beschrieben. Experimentelle Daten liegen insbesondere für Tantal vor und deuten auf um Größenordnungen höhere Diffusionskoeffizienten als die der linearen Extrapolation. Jedoch zeigen die mit verschiedenen Methoden bestimmten Diffusionskoeffizienten ebenfalls Abweichungen um mehrere Größenordnungen. Zum 
Vergleich eingezeichnet sind auch die oben gemachten Abschätzungen für die DDiffusonskoeffizienten in $\mathrm{V}$ und $\mathrm{Nb}$, die auf einen gemeinsamen Punkt fallen.

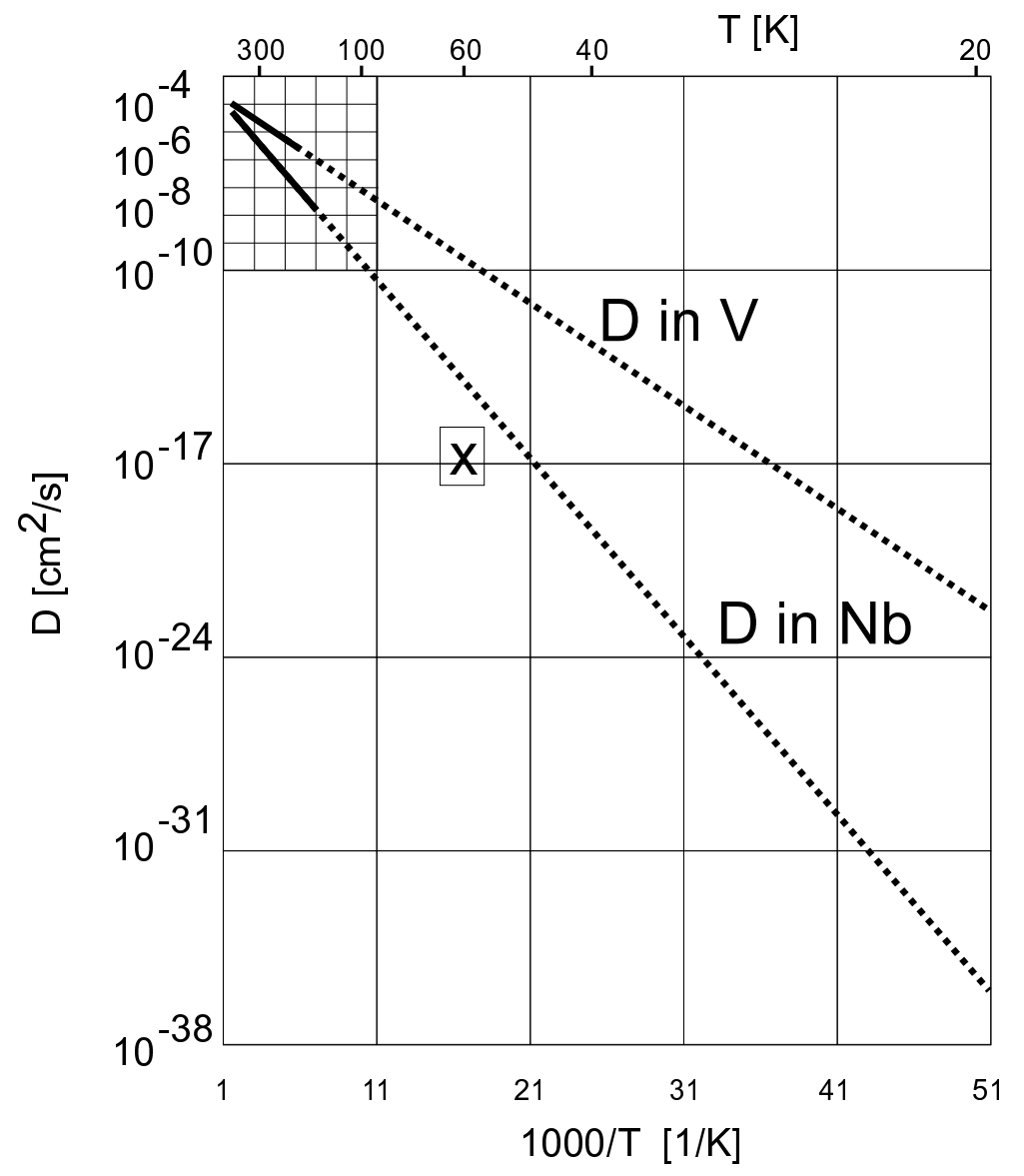

Abbildung 5.21: Diffusionskoeffizienten von Deuterium in V und Nb. Der durchgezogene Bereich der Kurven ist der Literatur entnommen [66]. Gestrichelt dargestellt ist die einfache Extrapolation dieser Daten zu Temperaturen von $20 \mathrm{~K}$. Als Kreuz eingezeichnet sind die Abschätzungen der Diffusionskoeffizienten aus den TAP-Messungen.

Entgegen den erwarteten höheren Werten im Vergleich zur Extrapolation, liegen die Punkte der einfachen Abschätzungen unterhalb dieser Linien. Daraus wird gefolgert, daß Diffusionskoeffizienten bei tieferen Temperaturen durch diese einfache Abschätzung nicht direkt zugänglich sind. Weitere zeitabhängige Messungen, wie sie am SIMS durchgeführt wurden (s. Abschnitt 5.2), könnten hier weiteren Aufschluß geben.

Festzuhalten ist, daß die gemessenen D-Profile bei $20 \mathrm{~K}$ keinen Einfluß einer Diffusion zeigen, so daß die Gleichgewichts-D-Verteilung detektiert werden kann. 


\subsection{Vergleich der H-/D-Segregation mit dem Mo- dell von Züchner}

Sowohl in den mit dem SIMS gemessenen H-Verteilungen, als auch in den mit dem TAP gewonnenen D-Verteilungen bei Temperaturen oberhalb von $20 \mathrm{~K}$ läßt sich ein Einfluß der H- bzw. D-Diffusion beobachten. Die Profile konnten qualitativ mit einem Modell beschrieben werden, das von einer Segregation des Wasserstoffs an den während der Messung geschaffenen Oberflächen ausgeht. In SIMSAnalysen an D-beladenen V-Folien finden Züchner et al. [26] ebenfalls eine erhöhte D-Konzentration in Oberflächenbereichen der Probe. Sie gehen von einer ausgedehnten Oberflächenphase aus, die durch den Ionenbeschuß der Probe während der SIMS-Messungen verursacht wird. Die in die Probe eindringenden Ionen führen demnach zu einer Aufweitung des Metallgitters in der Nähe der Oberfläche, so daß der Wasserstoff hier energetisch günstigere Plätze findet und eine H-reiche oberflächennahe Phase bildet. Die Ausdehnung dieser Oberflächenphase wird nicht angegeben.

Zwei Ergebnisse dieser Arbeit sprechen für die Annahme, daß der Einfluß der einfachen Besetzung freier Oberflächenplätze und sogenannter Subsurface-Sites $[67,68]$ höher ist, als der Einfluß der durch den Ionenbeschuß erhöhten Defektdichte unterhalb der Oberfläche.

1. In den TAP-Messungen bei $60 \mathrm{~K}$ wurde ebenfalls ein Anstieg der D-Konzentration zur Meßseite hin beobachtet (s. Abschnitt 5.5). Da bei der TAPAnalyse die Proben feldionisiert werden und nicht unter Ionenbeschuß stehen, fällt hier eine erhöhte Defektdichte unterhalb der Oberfläche als Ursache aus.

2. Die SIMS-Messungen an Pd/Nb-Schichten mit unterschiedlicher Abtragsgeschwindigkeit zeigen eine dem Modell von Züchner gegenläufige Oberflächensegregation (s. Abbn. 5.3 und 5.4). Die auf die Probe auftreffenden Ionen besitzen in der langsamen Messung eine geringere Ionenenergie als in der schnellen Messung, dadurch wird die geringere Abtragsrate erreicht. Die gemessenen Oberflächenkonzentrationen liegen jedoch höher als in der schnellen Messung, bei der durch die energiereicheren Ionen eine höhere Defektdichte auftreten sollte.

Diese Ergebnisse schliessen den Einfluß von Defektstrukturen auf die H-Verteilung in SIMS-Messungen nicht aus, jedoch zeigen sie, daß die gemachte Annahme einer erhöhten Belegung der Oberfläche ausreichend ist, um die gemessenen H- und DProfile zu verstehen. 


\subsection{Interpretation der bei $20 \mathrm{~K}$ gemessenen $\mathrm{D}$ - Verteilung}

In diesem Abschnitt sollen die dargestellten Ergebnisse der chemischen Tiefenprofilierung bei $20 \mathrm{~K}$ interpretiert werden. An den schon in Abschnitt 3 und 5.4.3 dargestellten Ergebnissen zeigte sich eine gleichmäßige D-Verteilung innerhalb der hochlösenden Schichten, die einer Gleichgewichtsverteilung zugeordnet wird. Um die Aussagekraft der gewonnenen Daten beurteilen zu können, soll zunächst die Diskussion der Fehlerquellen der dreidimensionalen Rekonstruktion der TAPDaten und der daraus gewonnenen Konzentrationsverläufe vorangestellt werden.

\subsubsection{Dreidimensionale Rekonstruktion der TAP-Daten und Konzentrationsbestimmung}

Die folgenden Analysen der TAP-Daten stützen sich auf die dreidimensionale Rekonstruktion der mit dem TAP gemessenen Daten. Wie in Abschnitt 3.1 beschrieben, liefert die TAP-Analyse sowohl das $\frac{e}{m}$-Verhältnis, als auch die laterale Position der detektierten Ionen. Die Sequenz der detektierten Ionen wird anschließend über geeignete Algorithmen in eine dreidimensionale Koordinate umgerechnet.

Alle Daten können nur mit einem gewissen Fehler angegeben werden. Der Fehler in der Massenbestimmung führt direkt zu einer Verbreiterung der detektierten Massen um die tatsächliche Masse. Die realisierte Massenauflösung $\frac{\Delta m}{m}$ liegt im Bereich von 0,01. Sie ist ausreichend, um die betrachteten Elemente zu unterscheiden. Die einzige Überschneidung ergab sich für $\mathrm{N}_{2}$ und das doppelt ionisierte Fe, die beide bei $\frac{e}{m}=28$ amu liegen. Da die $\mathrm{N}_{2}$-Konzentration in den anderen Elementen jedoch nicht über ein Prozent stieg, wurde sie auch im Fe vernachlässigt. Zudem konnte das Auftreten von Fe an Hand seiner Isotope stets zweifelsfrei erkannt werden.

In den Messungen der Nb-Schichten wurde auch NbD detektiert. Für die dreidimensionale Darstellung und die Bestimmung der D/Me-Konzentrationen wurden diese Signale in ein Nb- und ein D-Atom aufgeteilt. Als Position des Nb-Atoms wurde die gemessene Position des NbD-Ergeignisses beibehalten. Das D-Atom wurde im Abstand von einem per Zufallszahl auf die Kugelschale um diese Position platziert.

Die Positionsbestimmung besitzt jedoch durch die Kombination von Detektion und Rekonstruktion weitaus mehr Fehlerquellen [48]. Die Tiefenauflösung ist im Fall günstig orientierter Proben, bei denen die abgetragenen Kristallebenen senkrecht zur Meßrichtung liegen, gerade der Abstand der Ebenen. Die Auflösung ist somit atomar und wird auch erreicht wie Messungen an geordenten Legierungen zeigen. Beispiele hierfür sind auch die dargestellten Ebenen einer W-Spitze (Abb. 3.2) und die Nb- und D-Ebenen der beladenen Nb-Schicht (Abb. 5.9). Sind die Ebenen aus dieser idealen Orientierung verkippt, so sinkt die Tiefenauflösung, 
da die Atome nicht mehr lagenweise feldionisiert werden.

Während die Bestimmung des Auftreffortes auf dem Anodenfeld mit einer Genauigkeit von $0,5 \mathrm{~mm}$ gelingt, was bei Vergrößerungen von $10^{6}-10^{7} \mathrm{zu}$ theoretischen Auflösungen bis zu 0,5 auf der Probe führt, ist die laterale Projektion des Auftreffortes auf die Spitzenoberfläche weitaus unsicherer. Die für die Rekonstruktion verwendeten Projektionsmodelle gehen dazu von einem halbkugelförmigen Spitzenapex aus, an dem ein gleichmäßiges elektrisches Feld anliegt. An komplexen Proben wie Systemen mit Ausscheidungen und Schichtsystemen kann das lokale Feld jedoch beträchtlich von der zu Grunde liegenden Kugeloberfläche abweichen. Das liegt meist an den unterschiedlichen Feldverdampfungsfeldstärken verschiedener Phasen einer Probe. Eine Ausscheidung höherer Verdampfungsfeldstärke als die der umgebenden Matrix, wie z.B. MgO in einer Ag-Matrix [69] führt in der Spitze zu einem bevorzugten Abtrag der Matrix, was die Ausscheidung als Ort höherer Krümmung zurückläßt. Die verdampften Atome in diesem Bereich verlassen die Probe in einem größeren Winkelbereich, die Atome werden somit weiter gestreut und in der Rekonstruktion erscheint die Ausscheidung mit geringerer Dichte, da viele Atome außerhalb des Ausscheidungsvolumens detektiert werden.

Untersucht man Schichtsysteme, so tritt das Problem unterschiedlicher Verdampfungsfeldstärken an den Grenzflächen auf. Liegen die Schichten nicht senkrecht zur Meßrichtung, so führt die bevorzugte Verdampfung einer Komponente zu Verzerrungen in der Rekonstruktion. Trifft man beim Abtrag einer Schicht lokal auf die Grenzfläche zu einer Schicht mit geringerer Feldverdampfungsfeldstärke, so wird dieses zweite Element bevorzugt abgetragen. Der Rest der ersten Schicht wird erst später feldionisiert. Da der Rekonstruktionsalgorithmus von einem gleichmäßigem Ebenenabtrag und homogener Dichte auch über die Schichtgrenzen hinaus ausgeht und jedem detektiertem Atom einen festen Tiefenschritt zuordnet, führt die bevorzugte lokale Verdampfung zu hohen lokalen Dichten in der Rekonstruktion, während die restlichen Atome der ersten Schicht, die erst später detektiert werden, zu weit in die Tiefe platziert werden. Dies verringert die Tiefenauflösung im Bereich schrägliegender Grenzflächen.

Neben den unterschiedlichen Verdampfungsfeldstärken ist in den Schichtsystemen auch die Dichte der Metalle unterschiedlich. Dies ist die nächste Fehlerquelle, die aber nur für die dreidimensionale Darstellung der Daten und somit die Längenskalierung die entscheidene Rolle spielt. Während bei gleichmäßiger Verdampfung und senkrecht zur Schicht liegenden Schichtsystemen die relative Position der Atome gut rekonstruiert werden kann, wie man an den Beispielen erkennt, bei denen die Ebenen dargestellt werden können, hängt die absolute Längenskala von der angesetzten Dichte der Probe ab. Rekonstruiert man massive Proben, wie z.B. W, so macht man sicher keinen großen Fehler, wenn man für die Dichte den Literaturwert einsetzt. Im Rekonstruktionsalgorithmus kann bislang nur eine Dichte angeben werden, so daß auch bei der Rekonstruktion eines Schichtsystems alle Komponenten mit einer Dichte dargestellt werden. Es lassen 
sich unterschiedliche Komponenten somit nur einzeln mit der korrekten Dichte oder insgesamt mit einer mittleren Dichte darstellen. Die Dichte wird in Form des Atomvolumens in den Rekonstruktionsalgorithmus eingesetzt und bestimmt zusammen mit der Spitzengeometrie das rekonstruierte Volumen. Dies wirkt sich jedoch nicht auf die relative Auflösung im Bereich der Grenzflächen aus.

Alle Längenskalen hängen dadurch auch vom gewählten Atomvolumen ab. Bei den in dieser Arbeit durchgeführten Rekonstruktionen, die insbesondere der Untersuchung der Grenzflächen dienen sollen, wurde meist der Mittelwert der in Tabelle 3.1 angegebenen Atomvolumina eingesetzt. Für die beladenen Metallsysteme ergibt sich noch zusätzlich das Problem, daß das interstitiell gelöste Deuterium die Dichte verändert. In den Nb-Schichten, in denen eine hohe aber inhomogene D-Verteilung gemessen wurde, führt das zu unterschiedlichen Volumenrekonstruktionen im Hoch- und Tiefkonzentrationsbereich. Die Dichten wurden hier so gewählt, daß das Atomvolumen entsprechend der D-Konzentration reduziert wurde. So wurde in der Probe mit ca. $1 \mathrm{D} / \mathrm{Nb}$ die Hälfte des Wertes für $\mathrm{Nb}$ eingesetzt, da pro Nb-Zelle nun die doppelte Anzahl an Atomen detektiert wurde. Die zusätzliche Dehnung der Nb-Schichten durch die Beladung wurde in der angesetzten Dichte nicht berücksichtigt.

$\mathrm{Zu}$ diesen Faktoren kommt noch die in Abschnitt 3.1 erläuterte Bestimmung der Spitzengeometrie, die ebenfalls direkt in die Längenskala eingeht und eine Fehlerquelle darstellt, die mit der Dichtebestimmung vergleichbar ist. Insgesamt kann man die Qualität der dreidimensionalen Rekonstruktion der TAP-Daten und somit auch der daraus gewonnenen Konzentrationsanalysen wie folgt zusammenfassen:

- Die Auflösung der relativen Positionen der Atome zueinander ist in Bereichen gleichmäßiger Feldverdampfung nahezu atomar scharf, insbesondere gilt das für die Tiefenaufösung.

- In Schichtsystemen sind über die gesamte Messung solche guten Bedingungen selten gegeben. Die Rekonstruktion muß dann auf Teilbereiche der Messung abgestimmt werden, in denen die Grenzfläche senkrecht liegt. So kann auch hier eine nahezu atomare Schärfe erzielt werden.

- Die absolute Längenskala der Rekonstruktion ist durch die zahlreichen Fehlerquellen, wie Dichte und Geometrie der Probe, sehr unsicher. Es muß für die untersuchten Schichtsysteme mit einem relativen Fehler der rekonstruierten Längen von bis zu 50\% ausgegangen werden. Dies wirkt sich jedoch nicht auf die relative Auflösung im Bereich der Grenzflächen aus.

Da das TAP eine noch sehr junge Methode ist und die verwendeten Algorithmen zur Rekonstruktion insbesondere für Schichtsysteme sicher noch verbessert werden können, wird die heute schon einzigartige Ortsauflösung bei der chemischen Analyse von nanoskopischen Proben zukünftig sicher auch mit einer verbesserten absoluten Längenskalierung möglich sein. 
Nach der Fehlerbetrachtung der TAP-Analysen sollen nun die Ergebnisse der Messungen bei $20 \mathrm{~K}$ diskutiert werden.

\subsubsection{Diskussion der bei $20 \mathrm{~K}$ gemessenen D-Gleichge- wichtsverteilung}

Im Gegensatz zu den Messungen bei $60 \mathrm{~K}$ beobachtet man bei $20 \mathrm{~K}$ eine konstante D-Verteilung im Inneren der Nb- bzw. V-Schicht (s. Abbn. 5.14 und 5.20). Dies spricht für eine Unterdrückung des D-Transports zur Oberfläche des gemessenen Systems bei der gewählten Temperatur von $20 \mathrm{~K}$.

\section{Mittlere D-Konzentration in Nb- und V-Schichten}

Betrachtet man die gemessenen D-Konzentrationen, so fällt auf, daß sie mit 0,15 $\mathrm{D} / \mathrm{Nb}$ in der Nb-Schicht (Abb. 5.14) und mit ca. 0,09 D/V in der V-Schicht (Abb. 5.20) deutlich unterhalb der Werte liegen, die man bei einer Gasbeladung der massiven Metalle mit Drücken von 1,9 bzw. 6 mbar erwartet. Nach den (p,c)-Isothermen [31] erwartet man bei Raumtemperatur eine Beladung bis in die $\beta$-Phase der D-Metall-Systeme. Bei den entsprechenden tiefen Temperaturen entnimmt man dem Nb- bzw. V-H-Phasendiagramm (vgl. Abbn. 2.3 und 2.2), daß Hydridphasen mit Konzentrationen von mindestens $0,7 \mathrm{H} / \mathrm{Nb}$ bzw. 0,5 $\mathrm{H} / \mathrm{V}$ erwartet werden. Die Phasendiagramme der D-Metall-Systeme [40, 35] zeigen zwar leichte Abweichungen, die Isotopieeffekte können aber bei der Unsicherheit der Phasendiagramme für tiefe Temperaturen im Rahmen der oben gemachten Abschätzung vernachlässigt werden. Zudem werden für Dünnschichtsysteme und Multischichten Änderungen der Randlöslichkeiten und der Phasenbildung im Vergleich zu den massiven Metall-H-Systemen beobachtet, was im folgenden dargestellt werden soll.

Die D-Konzentrationen in den V-Systemen der Messung aus Abb. 5.20 von 0,09 D/V bei ca. $10 \mathrm{~nm}$ Schichtdicke und auch der Messung bei $60 \mathrm{~K}$ (Abb. 5.17), bei der Konzentrationen von ca. 0,08 D/V in ca. $5 \mathrm{~nm}$ dicken Schichten gemessen wurden, kann man mit Ergebnissen von Andersson et al. [17](Abb. 4a)) vergleichen, die an noch feiner modulierten Schichtpaketen gewonnen wurden. Sie finden in Fe/V-Übergittern mit V-Schichtdicken von 1,6 nm bei einer Beladung mit weniger als 5 mbar $\mathrm{H}_{2}$-Gaspartialdruck $\mathrm{H}$-Konzentrationen, die unterhalb von 0,2 $\mathrm{H} / \mathrm{V}$ liegen. Desweiteren messen sie eine Absenkung der kritischen Temperatur zur Bildung der $\beta-\mathrm{H}-\mathrm{V}$-Phase unter $25^{\circ} \mathrm{C}$. Zwar sind die in dieser Arbeit untersuchten Schichtdicken größer, die gefundenen D/V-Konzentrationen liegen aber im Bereich der zitierten Werten. Berücksichtigt man, daß die verwendeten Drücke geringer waren und auch, wie unten angesprochen, ein D-Verlust an die Gasphase nicht ausgeschloßsen werden kann, so wird die Übereinstimmung eher besser.

Es ist nicht auszuschließen, daß die untersuchten Schichtsysteme im Verlauf des Transfers von der Beladungskammer ins TAP und innerhalb der UHV- 
Kammer Deuterium an die Gasphase abgeben und dadurch niedrigere Konzentrationen aufweisen, als es dem Beladungsdruck entspräche. Durch die Verwendung von Pd als Deckschicht ist ein D-Austausch mit der Gasphase der Umgebung möglich, da eine Oxidschicht nicht gebildet werden kann. Die Abhängigkeit der D-Konzentration von der Beladung und der Schichtdicke kann somit nicht exakt bestimmt werden. Festzuhalten ist jedoch, daß durch die gemessenen DKonzentrationen ein vollständiges Ausgasen der Proben ausgeschlossen werden kann.

Dem konstanten Verlauf der D-Konzentration zufolge werden in den V-Schichten bei der Abkühlung der Proben keine Anzeichen für eine Entmischung beobachtet. Auch in der Messung bei $60 \mathrm{~K}$ (Abb. 5.17) wird im Gegensatz zu den Messungen der Nb-Schichten (s. Abschnitt 5.5) kein Bereich erhöhter DKonzentration gemessen, der einer $\beta$-Phase zugeordnet werden könnte. Die am Anfang der Schichten erhöhte Konzentration von ca. 0,15 D/Me liegt weit unterhalb der Hydridkonzentrationen. Der gleichmäßige Abfall innerhalb der Schichten wird demzufolge der Diffusion zugerechnet.

Als Ursache dafür, daß die Entmischung nicht beobachtet wird, wäre einerseits denkbar, daß die kritische Temperatur in den dünnen Schichten weit unterhalb von $25^{\circ} \mathrm{C}$ läge. Dagegen spricht, daß die Schichtdicke von $10 \mathrm{~nm}$ der Schicht, die bei $20 \mathrm{~K}$ gemessen wurde, eher groß ist und ein starker Dünnschichteffekt auf die kritische Temperatur eigentlich nicht erwartet wird. Eine zweite Möglichkeit wäre, daß die Bildung der Hydridphase bei tiefen Temperaturen kinetisch unterdrückt wäre. Drittens könnten Spannungen in den Schichten die Randlöslichkeit des Systems erhöhen, wie von Laudahn [7] in Nb-Schichtsystemen beobachtet wurde. Eine weitere Möglichkeit wäre eine geringere Detektionswahrscheinlichkeit der D-Ionen im TAP im Vergleich zu der der V-Ionen. Dagegen spricht jedoch die Detektion der maximalen Konzentrationen von 1 D/Nb im Nb-D-System (Abb. $5.9)$.

Als Ergebnis läst sich für die V-Schichten festhalten: Sowohl die ca. $10 \mathrm{~nm}$ dicke V-Schicht als auch die $5 \mathrm{~nm}$ dicken V-Schichten befinden sich bei detektierten Konzentrationen von 0,09 bzw. 0,08 D/V in der $\alpha$-Phase des V-DDünnschichtsystems. Es wird somit bei Temperaturen von bis zu $20 \mathrm{~K}$ eine homogene Löslichkeit von 0,09 D/Me gemessen. Ob dies ein Effekt der Spannungen in dünnen Schichten oder eine kinetische Unterdrückung der Phasenbildung ist, könnte durch weitere Messungen an V-Schichten unterschiedlicher Schichtdicken bei $20 \mathrm{~K}$ untersucht werden.

Die gemessene D-Konzentration in der Nb-Schicht von 0,15 D/Nb (Abb. 5.14) liegt ebenfalls deutlich unterhalb der Konzentration des Nb-Hydrides. Auch in dünnen Nb-Schichten findet man eine Absenkung der kritischen Temperatur. So erwarten Song et al. [4] eine Absenkung der kritischen Temperatur auf 0 K unterhalb einer Schichtdicke von $4 \mathrm{~nm}$. Eine Entmischung innerhalb der Nb-Schicht wäre für ein solches Schichtsystem nicht mehr zu erwarten.

Die Absenkung der kritischen Temperatur führt gleichzeitig zu einer Erhöhung 
der Randlöslichkeit von Wasserstoff bzw. Deuterium in Nb. So findet Laudahn [7] sowohl in laserdeponierten Nb-Einzel- als auch Pd/Nb-Multischichtsystemen Randlöslichkeiten von bis zu 0,26 H/Nb. Allerdings führt Laudahn dies auf den Einfluß von Spannungen zurück, da epitaktische Schichten, die leicht plastisch verformen und geringe Spannungen aufweisen, die H-Löslichkeit des massiven $\mathrm{Nb}$ aufweisen. Da die gemessene Nb-Schicht eine sehr geringe Dicke von ca. 2,5 $\mathrm{nm}$ hatte, sind alle angesprochenen Effekte auf die Löslichkeit denkbar. Dies führt zu dem Schluß, daß auch der mit dem TAP an der Nb-Schicht bei $20 \mathrm{~K}$ gemessene Volumenbereich sich wie die V-Schichten in der $\alpha$-Phase des D-MeSystems befand.

Die Ergebisse der Messungen der Nb-Schichten, die bei $60 \mathrm{~K}$ durchgeführt wurden und bei denen zumindest in Teilbereichen der Schicht das Hydrid gemessen werden konnte, schließen jedoch nicht aus, daß sich auch die Nb-Schicht mit 0,15 D/Nb im Zweiphasengebiet befunden hat. So könnte eine Hydridbildung in Bereichen der Probe stattgefunden haben, die mit der Analysefläche des TAP nicht erfaßt wurden.

\section{D-Konzentration an der Grenzfläche}

Die vorhergehenden Betrachtungen bezogen sich alle auf die D-Konzentrationen innerhalb der hochlösenden $\mathrm{Nb}$ - und V-Schichten. Sie ergaben, daß für die Messungen bei $20 \mathrm{~K}$ von einer Abbildung der Gleichgewichtsverteilung ausgegangen werden kann. Deswegen soll im folgenden an den entsprechenden Profilen (Abbn. 5.14 und 5.20) auf den Verlauf der D-Konzentration an den Grenzflächen eingegangen werden. Hinzuziehen läßt sich außerdem die Messung des Fe/V-Systems bei $60 \mathrm{~K}$ (Abb. 5.17), die ein abfallendes D-Profil innerhalb der V-Schichten zeigt. Die D-Konzentrationen an den hinteren V/Fe-Grenzflächen liegen zwar durch die D-Diffusion an die Oberfläche unterhalb des mittleren Wertes, jedoch kann der Abfall der reduzierten Konzentration von ca. $0,04 \mathrm{D} / \mathrm{V}$ im $\mathrm{V}$ bis auf $0 \mathrm{D} / \mathrm{V}$ im Fe ebenfalls Aussagen liefern.

Wie in Abschnitt 2.2 dargestellt, sind für das Lösungsverhalten von Wasserstoff an metallischen Grenzflächen mehrere Varianten denkbar (vgl. Abb. 2.1). Diese folgen direkt aus der Struktur der Grenzfläche der Metalle. An Metallgrenzflächen mit atomar scharfer Grenzfläche werden elektronische Übergangsstrukturen und ein Elektronenübertritt ins elektronegativere Metall erwartet [20, 16], der sich durch eine reduzierte H-Löslichkeit an der Grenzfläche in der H-affineren Komponente auswirken sollte (Abb. 2.1b)). Auf der anderen Seite ist aber eine H-Verarmung an der Grenzfläche auch zu erwarten, wenn es an der Grenzfläche zu einer chemischen Durchmischung kommt (Abb. 2.1c)), da die H-Löslichkeit in binären Legierungen i.A. mittlere Werte der Reinelemente annimmt, wie aus Bandstrukturrechnungen von Griessen et al. [32] hervorgeht. Die durchgeführten TAP-Messungen erlauben eine gleichzeitige Bestimmung der Konzentrationsverläufe der Metalle und des Deuteriums an der Grenzfläche [70]. 
Die gefundenen Verläufe an $\mathrm{Nb} / \mathrm{W}$ und $\mathrm{V} / \mathrm{Fe}-$ Grenzflächen zeigen das folgende einheitliche Bild: Die innerhalb der hochlösenden Nb- bzw. V-Schichten hohen D-Konzentrationen nehmen über die Grenzfläche kontinuierlich ab und verschwinden in der gering lösenden Komponente W bzw. Fe. Dabei wird eine simultane Abnahme der Konzentrationen des Deuteriums und des hochlösenden Metalls gemessen.

Einzig an der Pd/V-Grenzfläche der Messung bei $20 \mathrm{~K}$ (Abb. 5.20) zeigt sich ein Abfall der D-Konzentration schon innerhalb der V-Schicht. Diese Verarmung wird später diskutiert. Es folgt zunächst die Interpretation der Ergebnisse an den Grenzflächen Nb/W und V/Fe, die sich durch einen sehr großen H-Löslichkeitsunterschied auszeichnen.

\section{$\mathrm{Nb} / \mathrm{W}$ - und V/Fe-Grenzflächen}

Zur Beurteilung, ob die Abnahmen auf elektronische oder chemische Effekte zurückgeführt werden können, muß man den Verlauf der Metallkonzentrationen näher untersuchen. Dazu wurde für die Nb- und V-Schichten der Bereich bestimmt, in dem Nb bzw. V einen Anteil von über 90\% am Metall besitzen. Dieser Bereich ist in den Abbildungen der Konzentrationsprofile durch die waagerechte rote Linie gekennzeichnet, die gleichzeitig auch die mittlere D-Konzentration in diesem Bereich anzeigt. Als Breite der Durchmischungszone wird der Bereich der Grenzfläche angenommen, in dem keines der angrenzenden Metalle mit einer Konzentration über $90 \%$ detektiert wird. In diesem Bereich kann von einer meßbaren chemischen Durchmischung ausgegangen werden. Eine D-Verarmung durch elektronische Effekte sollte sich auch innerhalb der Schicht zeigen. Bis auf die angesprochene $\mathrm{Pd} / \mathrm{V}$-Grenzfläche zeigen alle D-Profile im Bereich der reinen Nbbzw. V-Schichten konstant hohe Werte. Erst in den durchmischten Bereichen sinken die D-Konzentrationen und zwar simultan mit der Nb- bzw. V-Konzentration. Die D-Verarmung wird somit auf der gleichen Längenskala beobachtet, auf der auch die detektierte Grenzflächenschärfe der Metalle liegt.

Die Durchmischung an der Grenzfläche nimmt an der Nb/W-Grenzfläche eine Breite von ca. 0,6 nm ein (Abb. 5.14). Bei den V/Fe- Grenzflächen wird eine chemische Unschärfe von ca. $1 \mathrm{~nm}$ in der Messung bei $60 \mathrm{~K}$ (Abb. 5.17) und von knapp $2 \mathrm{~nm}$ in der Messung bei $20 \mathrm{~K}$ (Abb. 5.20) beobachtet. Die absoluten Längen sind wegen der in Abschnitt 5.7.1 angesprochenen Dichtemittelung der Rekonstruktion eher zu hoch angegeben. Als Aussage läßt sich jedoch festhalten, daß das D-Konzentrationsprofil simultan zu den Änderungen der Nb- bzw. VKonzentration verläuft. An der Nb/W- und der V/Fe-Grenzfläche kann somit eine D-Verarmung, die von elektronischen Grenzflächeneffekten stammt, nicht von der gefundenen Verarmung im Durchmischungsgebiet getrennt werden.

$\mathrm{Zu}$ beachten ist jedoch, daß die gefundenen Durchmischungszonen von 0,6 - 2 $\mathrm{nm}$ breiter sind als die von Andersson et al. [17] an Fe/V-Schichten gefundenen Verarmungszonen. Sie berichten von einer Ausdehnung der Dead Layer über 3 Mo- 
nolagen, was bei den untersuchten (100)-Fe/V-Übergittern auf MgO-Einkristallen mit Schichtdicken von 0,9 - 2,0 nm einer Breite von 0,45 nm entspräche. Gleichzeitig geben sie für die Grenzflächendurchmischung eine Breite von ca. 0,2 nm an, die mit Klein- und Großwinkel-Röntgendiffraktometrie bestimmt wurde [39]. Für Schichtdicken von 0,9 nm finden sie eine verschwindene H-Konzentration, die Schicht wird ihrem Modell zufolge von den zwei Dead-Layern mit Einzelbreiten von $0,45 \mathrm{~nm}$ ausgefüllt.

Betrachtet man die angegebene Grenzflächendurchmischung von $0,2 \mathrm{~nm}$ so entspricht auch diese Zone einer Breite von 0,4 nm. Die ungestörte Schicht ist somit nur noch $0,5 \mathrm{~nm}$ breit. Ein Einfluß dieses auch nicht vernachlässigbaren Bereichs der Grenzfläche der Fe/V-Schichten auf die Löslichkeit wird aber nicht diskutiert.

Da absolute Längenangaben der mit dem TAP untersuchten Schichten nicht möglich sind, ist auch eine absolute Quantifizierung der Grenzflächenschärfe nicht möglich. Insbesondere im Fall der Nb/W-Grenzfläche, die mit 4,4\% eine geringere Gitterfehlpassung besitzt als das System Fe/V (5,5\%), ist aber davon auszugehen, daß die Qualität der Grenzfläche mit denen der epitaktischen Übergitter von Andersson et al. vergleichbar ist. Dafür sprechen die Ergebnisse der Feldionenmikroskopie an der Nb/W-Grenzfläche (s. Abb 4.6), die zeigen, daß die Nb-Schicht lokal epitaktisch auf dem W-Substrat aufwächst. Zudem zeigen die Konzentrationsanalysen eine Durchmischungszone von ca $0,6 \mathrm{~nm}$, die somit in den Bereich von 2 Monolagen im (110)-Nb kommt.

Da das System Nb/W für die massiven Metalle einen um fast 15 Größenordnungen größeren Unterschied in der H-Löslichkeit zeigt als V/Fe (vgl. Tab. 2.4), sollte ein elektronischer Grenzflächeneffekt hier noch deutlicher ausfallen. Daß dieser nicht beobachtet wird, ist ein weiterer Hinweis auf einen geringeren Einfluß elektronischer Effekte auf die H-Löslichkeit an metallischen Grenzflächen als er von Hjörvarsson et al. vorgeschlagen wird.

Ein weiterer Punkt, der in den Arbeiten von Hjörvarsson et al. nicht diskutiert wird, ist der Einfluß der kernphysikalischen $\mathrm{N}^{15}$-Untersuchungsmethode auf die detektierten H-Konzentrationen in den Multischichtsystemen. Auch bei der $\mathrm{N}^{15}$-Methode wird die Probe mit hochenergetischen Ionen beschossen $(>6$ $\mathrm{MeV}$ ). Auftretende Absputtereffekte an der Probenoberfläche könnten je nach Ausmaß durch den in Abschnitt 5.3 beschriebenen Diffusionsmechanismus signifikante Wasserstoffmengen an der Oberfläche binden. Dies würde dann zu Messungen von reduzierten H-Konzentrationen innerhalb der Probe führen. Da die Breite der Dead-Layers aus der detektierten mittleren H-Konzentration berechnet wird [16], könnte die Breite der Dead-Layers überschätzt werden.

Untersuchungen mit dem TAP an den von Hjörvarsson hergestellten planaren Schichtsystemen könnten zur weiteren Klärung des Einflusses elektronischer Effekte beitragen. 


\section{Pd/V- und Pd/Nb-Grenzflächen}

Bei den untersuchten $\mathrm{Pd} / \mathrm{V}$ - und $\mathrm{Pd} / \mathrm{Nb}$-Grenzflächen muß das kubisch flächenzentrierte Pd auf eine kubisch raumzentrierte Struktur aufwachsen. Dies und der große Gitterparameter von Pd führen zu einer starken Gitterfehlpassung der beteiligten Metallgitter (s. Tab. 2.3). In der Ebene muß sich das Pd mit seinen $\left[\frac{1}{2} \frac{1}{2} 0\right]$-Richtungen an den [100]- und den $\left[\frac{1}{2} \frac{1}{2} \frac{1}{2}\right]$-Vektor im krz-Metall anpassen. Nach den Daten der massiven Metalle ergeben sich im Fall der V/Pd-Grenze 10 und $-4,7 \%$ und beim Nb/Pd 20 und $4 \%$ Fehlpassung. Beim Aufwachsen von Pd auf $\mathrm{V}$ muß eine Richtung gedehnt und eine gestaucht werden, wobei die $10 \%$ ige Fehlpassung durch eine Dehnung des Pd-Gitters in der Grenzfläche ausgeglichen werden muß. Die V-Schichten sind demzufolge in der Schichtebene gestaucht.

Ein Aufwachsen von Pd auf V oder Nb sollte demnach zum Einbau von Versetzungen zum Ausgleich dieser Fehlpassung führen. So finden Isberg et al. [39] mit Kleinwinkel-Röntgendiffraktometrie Hinweise auf eine hohe Versetzungsdichte an der Pd/V-Grenzflächen.

Ein weiterer Hinweis auf entsprechend hohe Versetzungsdichten an der Grenzfläche liefert die Instabilität der $\mathrm{Pd} / \mathrm{Nb}$-Grenzflächen bei den feldionenmikroskopischen Untersuchungen. Während die Nb/W-Grenzfläche den starken Hochspannungsfeldern zunächst standhielten, rissen die Pd/Nb-Schichtsysteme an der Grenzfläche ab, insbesondere wenn durch eine D-Beladung der Nb-Schichten, die mechanischen Spannungen in der Schicht noch erhöht waren.

Den dritten Hinweis auf eine erhöhte Defektdichte an den Pd/Nb-Grenzflächen liefern die im Anhang A beschriebenen Ergebnisse der H-Permeationsmessungen an $\mathrm{Pd} / \mathrm{Nb}-\mathrm{Multischichten.} \mathrm{Sie} \mathrm{zeigen} \mathrm{eine} \mathrm{sehr} \mathrm{stark} \mathrm{verzögerte} \mathrm{H-Permeation} \mathrm{im}$ Vergleich zur Verzögerung, die man nach dem Permeationsmodell [61] für ein periodisches Schichtsystem ohne Grenzflächeneffekte und mit den Diffusions- und Löslichkeitseigenschaften von Wasserstoff in massiven Metallen erwartet. Diese Verzögerung kann durch Modifikation des Modells auf Dünnschicht- und Grenzflächeneffekte hin untersucht werden.

So kann die experimentell bestimmte Verzögerung im Modell einerseits simuliert werden, wenn mit abnehmender Schichtdicke die Löslichkeit in den NbSchichten ansteigen würde. Für die untersuchten Nb-Schichten mit Dicken von $33,5 \mathrm{~nm}, 13 \mathrm{~nm}$ und 8,5 nm steigen die gefundenen Löslichkeitsunterschiede bezogen auf $\mathrm{Pd}$ (vgl. Abb. A.4) von ca. $2 \cdot 10^{6}$ für 33,5 nm dicke Nb-Schichten in der Probe mit 4 Doppelschichten auf $6 \cdot 10^{6}$ für $8,5 \mathrm{~nm}$ dicke Nb-Schichten in der Probe mit 16 Doppelschichten. Damit liegen die Werte um ca. 3 Größenordnungen über dem Wert von $3 \cdot 10^{4}$ für massives $\mathrm{Nb}$ (s. Tab. 2.4).

Andererseits kann die experimentell bestimmte Verzögerung durch die Einführung einer Zwischenschicht zwischen den Pd- und Nb-Schichten simuliert werden, wenn diese Zwischenschichten eine höhere H-Löslichkeit besitzen als die NbSchichten (s. Abb. A.6). Eine $1 \mathrm{~nm}$ dicke Zwischenschicht an allen Grenzflächen kann im Modell die Permeation simulieren, wenn die Zwischenschicht einen Lös- 
lichkeitsunterschied von ca. $4 \cdot 10^{7}$ bezogen auf Pd besitzt. Durch die Einführung dieser Grenzflächenmodifikation, die die Eigenschaften innerhalb der Pd- und Nb-Schichten unverändert läßt, liefert das Modell mit einem für alle 3 Proben gemeinsamen Wert für die Löslichkeit in der Zwischenschicht eine Übereinstimmung mit dem Experiment.

Dies spricht für einen Grenzflächeneffekt in den $\mathrm{Pd} / \mathrm{Nb}$-Schichten, der mit einer erhöhten H-Löslichkeit an der Grenzfläche verbunden ist. Nach den Überlegungen zu möglichen Grenzflächeneffekten (s. Abb. 2.1) führen sowohl die von Hjörvarsson vorgeschlagenen elektronischen Effekte als auch Effekte chemischer Durchmischung zu verringerter H-Löslichkeit an der Grenzfläche. Eine erhöhte Defektdichte an den Grenzflächen hingegen erhöht die H-Löslichkeit. Somit sind die Ergebnisse der H-Permeation ein weiteres Indiz für eine erhöhte Defektdichte insbesondere durch den Einbau von Anpassungsversetzungen an den $\mathrm{Pd} / \mathrm{Nb}$ Grenzflächen zum Ausgleich der hohen Gitterfehlpassung.

Vor dem Hintergrund, daß die Pd/V-Grenzfläche strukturell von den Nb/Wund $\mathrm{Fe} / \mathrm{V}$-Grenzflächen abweicht, soll die in der Messung am $\mathrm{Pd} / \mathrm{V} / \mathrm{Fe}-\mathrm{Schich}$ paket beobachtete D-Verarmung an der Pd/V-Grenzfläche (s. Abb. 5.20) im folgenden erörtert werden.

Der Anstieg der D-Konzentration erfolgt im Vergleich zum Anstieg der VKonzentration um ca. $2 \mathrm{~nm}$ verzögert. Dies könnte als elektronischer Effekt gedeutet werden. Gegen diese Annahme spricht jedoch die Tatsache, daß gleichzeitig an der auf die $\mathrm{Pd} / \mathrm{V}$-Grenze folgenden V/Fe-Grenzfläche keine D-Verarmung beobachtet wird. Sind elektronische Effekte für die D-Verarmung ausschlaggebend, so sollte sich an der V/Fe-Grenzfläche, die einen um sechs Größenordnungen höheren Löslichkeitsunterschied besitzt (vgl. Tab. 2.4), ein weit grösserer Effekt einstellen. Da exakt das Gegenteil beobachtet wird, muß diese D-Verarmung eine andere Ursache haben.

Eine mögliche Erklärung könnte die angesprochene Struktur der Grenzfläche liefern. So führt die oben angesprochene Anpassung des Pd- an das V-Gitter zu einer Stauchung des V-Gitters in der Grenzfläche. Dies könnte zu einer Reduzierung der Platzvolumina im Zwischengitter des V in der Nähe der Grenzfläche führen. Somit würden diese Plätze für Deuterium energetisch ungünstiger, was zu einer D-Verarmung an der Grenzfläche führt.

Dem entgegenwirken kann jedoch der Einbau von Versetzungen an der Grenzfläche zum Abbau der Anpassungsspannungen. Zum einen relaxiert das Gitter, so daß im V wieder mehr Deuterium in der Nähe der Grenzfläche gelöst werden kann, und zum anderen führen die Versetzungen auch selbst zu Veränderungen der Platzenergieverteilungen für D. Wie in Abb. 2.1d) skizziert, würde das Dilatationsfeld von Versetzungen, wenn es in die V-Schicht reicht, zu einer bevorzugten Besetzung dieser Grenzfächenplätze führen. Im umgekehrten Fall ist aber auch denkbar, daß Versetzungen in der Grenzfläche so orientiert sind, daß ihr Kompressionsfeld in die V-Schicht gerichtet ist. In diesem Bereich wäre das Gitter verengt und die Löslichkeit würde absinken. 
Einen bislang ungeklärten Einfluß auf die Grenzflächenstruktur, könnte auch die Depositionstemperatur haben. Die betrachtete Pd/V-Schicht wurde bei Temperaturen weit oberhalb von $320^{\circ} \mathrm{C}$ hergestellt. Dies kann man in der Konzentrationsanalyse (Abb. 5.20) an den Grenzflächen erkennen, die mit einer Durchmischungszone von ca. $2 \mathrm{~nm}$ durch einsetzende Interdiffusion unschärfer als bei den bei RT hergestellten Systemen waren.

Eine sichere Aussage, welcher Mechanismus die Ursache für die beobachtete D-Verarmung ist, kann zum jetzigen Zeitpunkt nicht gegeben werden. Weitere Messungen zur Bestimmung der Grenzflächenstruktur (z.B. mittels HREM) und der mechanischen Spannungen von Pd/V-Schichten könnten weitere Informationen liefern. 


\section{Kapitel 6}

\section{Zusammenfassung}

Ausgangspunkt für die durchgeführten Untersuchungen war die Frage nach der lokalen Wasserstoffverteilung in metallischen Multischichtsystemen, angestoßen durch ein von Hjörvarsson et al. [16] gefundenes Grenzflächenphänomen, den sogenannten Dead-Layers. Ziel war es, hierfür die zur Verfügung stehenden hochauflösenden Methoden der tomographischen Atomsonde (TAP) und der Sekundärionenmassenspektrometrie (SIMS) zur chemischen Analyse der Metall-H-Systeme auf Nanometerskala einzusetzen. Dabei sollte einerseits geklärt werden, ob die Methoden eine quantitative chemische Analyse der Metall-H-Systeme auf Nanometerskala zulassen. Andererseits sollte bei einem erfolgreichem Einsatz der Methoden untersucht werden, ob eine H-Verarmung an Grenzflächen von Schichten mit stark unterschiedlicher H-Löslichkeit auch lokal gemessen werden kann. Bisher war mit Hilfe der kernphysikalischen $\mathrm{N}^{15}$-Methode nur eine Bestimmung der HKonzentration als Mittelwert über mehrere Schichten unter Einbeziehung der geringlösenden Schichten möglich.

Die Ergebnisse der Untersuchungen mit den hochauflösenden Meßmethoden und ihre Interpretation in Bezug auf die lokale Wasserstoffverteilung in metallischen Multischichtsystemen lassen sich wie folgt zusammenfassen:

Es wurden $\mathrm{Pd} / \mathrm{Nb}$ - und Fe/V-Schichtsysteme auf W und Si mit Ionenstrahlsputterdeposition und gepulster Laserdeposition hergestellt.

Sowohl mit dem SIMS, als auch mit dem TAP lassen sich die metallischen Multischichtsysteme mit Schichtdicken im Nanometerbereich chemisch analysieren. Durch die hohe Tiefenauflösung von ca. 0,5 nm gelingt es mit dem TAP, den Bereich der Grenzflächen in seiner chemischen Zusammensetzung im Subnanometerberech zu analysieren. Die geringere Auflösung des SIMS unter den für die Detektion der Metallcäside optimierten Analysebedingungen und die durch die Primärionen ausgelöste Durchmischung der Schichten erlaubt hingegen nur eine chemische Analyse der Grenzflächen mit einigen Nanometern Genauigkeit.

Die Wasserstoff- bzw. Deuterium-Konzentration in den Multischichten konnte quantitativ bestimmt werden. Es konnte gezeigt werden, daß Wasserstoff bzw. Deuterium selbst bei $60 \mathrm{~K}$ noch so mobil ist, daß die gemessenen Konzentra- 
tionsprofile auf Diffusionsprozesse von Wasserstoff während der Messung zurückzuführen sind. Dies läßt sich wie folgt interpretieren: Da bei den Analysemethoden während der Messung die Oberfläche sukzessive abgetragen wird, bieten sich dem Wasserstoff an der jeweils "frischen" Oberfläche energetisch günstigere Plätze, sogenannte Surface- und Subsurface-Plätze, deren Besetzung einen H-Fluß zur Oberfläche nach sich zieht. Die Berücksichtigung einer erhöhten Defektdichte an der Oberfläche, wie sie von Züchner für SIMS-Messungen als Erklärung herangezogen wird [26], ist zur Beschreibung des Effektes nicht notwendig. Daß der Effekt auch an TAP-Messungen gefunden werden konnte, bei denen die Proben nicht unter Ionenbeschuß stehen und somit keine erhöhten Defektdichten existieren, zeigt den wesentlichen Einfluß der Oberflächenplätze. Die lokale WasserstoffGleichgewichtsverteilung in den Multischichten ohne den Einfluß der Wasserstoffdiffusion ist damit erst bei tiefsten Temperaturen von ca. $20 \mathrm{~K}$ meßbar.

Mit dem TAP konnten D-beladene Nb/W- und V/Fe-Grenzflächen bei $20 \mathrm{~K}$ analysiert werden. Es wurde in allen Fällen eine Abnahme der D-Konzentration vom $\mathrm{Nb}$ bzw. $\mathrm{V}$ zur Grenzfläche hin gemessen, die simultan mit der Abnahme der Nb- bzw. V-Konzentration verläuft. Eine D-Verarmung wird somit nur als Folge der chemischen Durchmischung der Grenzfläche festgestellt. Ein DeadLayer als Konsequenz eines Ladungsübertrages zwischen den Schichten, d.h. eine D-Verarmung, die auch in den Teil der Nb- bzw. V-Schichten hereinreicht, der chemisch nicht durchmischt ist, wurde nicht gefunden.

Das wird besonders deutlich an den Ergebnissen der Nb/W-Grenzfläche, die lokal epitaktisch hergestellt werden konnte und an der keine D-Verarmung detektiert wurde. Der H-Löslichkeitsunterschied liegt hier um viele Größenordnungen über dem des V/Fe-Systems. Somit sollte der von Hjörvarsson et al. [16] vorgeschlagene elektronische Effekt noch deutlicher als im V/Fe-System auftreten.

Das Resultat schließt jedoch die Existenz der Dead-Layers in Fe/V-Schichten nicht abschließend aus. Die untersuchten Fe/V-Schichtsysteme ließen sich aufgrund der geringen Schichtdicken im FIM nicht bezüglich Ihrer kristallographischen Orientierung und Korngrößen untersuchen. Ein direkter Vergleich der Schichtqualität mit den von Andersson et al. [17] untersuchten Fe/V-Schichten, bei denen Dead-Layers von drei Monolagen Dicke gefunden wurden, ist somit nicht möglich.

Untersuchungen an $\mathrm{Pd} / \mathrm{Nb}$ - und $\mathrm{Pd} / \mathrm{V}$-Grenzflächen deuten auf Versetzungsstrukturen hin, die an der Grenzfläche eingebaut werden, um die im Vergleich zu den $\mathrm{Nb} / \mathrm{W}$ - und V/Fe-Grenzflächen weit größere Gitterfehlpassung der Metalle auszugleichen. So zeigen Messungen an Pd/Nb-Schichten mit dem TAP eine hohe mechanische Instabilität. Dies wird durch die Ergebnisse der elektrochemischen Permeationsmessungen und deren Modellierung unterstützt. Die stark verzögerte H-Permeation durch Pd/Nb-Multischichten kann mit dem Permeationsmodell [61] gut simuliert werden, wenn Zwischenschichten hoher H-Löslichkeit, wie sie durch eine erhöhte Defektdichte an den Grenzflächen verursacht wird, eingeführt werden.

Zusammenfassend läßt sich sagen, daß die lokale Wasserstoffverteilung in me- 
tallischen Multischichten mit der tomographischen Atomsonde sowie mit der Sekundärionenmassenspektrometrie quantitativ meßbar ist. Eine Korrelation mit der lokalen Verteilung in den Multischichten ohne den Einfluß von Diffusionsprozessen des Wasserstoffs ist bei den oberflächenabtragenden Methoden aber nur möglich, wenn die Beweglichkeit des Wasserstoffs eingefroren ist. Hierzu werden abhängig vom untersuchten Metall-Wasserstoff-System tiefe Temperaturen von ca. $20 \mathrm{~K}$ benötigt. 


\section{Anhang A}

\section{Elektrochemische Bestimmumg der H-Permeation durch Pd/Nb-Multischichten}

Im folgenden soll kurz die elektrochemische Bestimmung der H-Permeation beschrieben und anschließend Ergebnisse an $\mathrm{Pd} / \mathrm{Nb}-\mathrm{Multischichten}$ dargestellt werden. Die Messungen wurden als Fortsetzung der in [64, 61] beschriebenen Experimente durchgeführt, um den Einfluß der Grenzflächen auf die H-Permeation in Multischichten näher zu untersuchen.

Durch das in Abschnitt 5.3.1 beschriebene Modell zur H-Permeation durch Multischichten [61] ist es möglich, experimentelle Daten mit modellierten zu vergleichen. In das Modell gehen neben den Schichtdicken $a_{r}$ die Diffusionskoeffizienten innerhalb der Einzelschichten $D_{r}$ sowie die H-Löslichkeiten der Metalle $k_{r}$ ein. Wie in Abschnitt 5.3.1 beschrieben, führt die abwechselnde Anordnung von Metallen mit großem H-Löslichkeitsunterschied $\frac{k_{r}}{k_{r+1}}$ zu großen Verzögerungen in der H-Permeation. Dies erhält man schon aus der Modellierung mit den Literaturdaten für D und k (s. Tabn. 2.2 und 2.4) und der Annahme, daß die Schichten periodisch und ohne Grenzflächeneffekte aufgebaut sind (vgl. Abb. 2.1 Fall a).

In realen Schichtsystemen auftretende Grenzflächeneffekte als Folge elektronischer Effekte, chemischer Durchmischung oder erhöhten Defektdichten an den Grenzflächen (vgl. Abb. 2.1 Fall b, c und d) sollten zusätzlichen Einfluß auf die H-Permeation haben.

Diese Grenzflächeneffekte lassen sich mit dem Modell ebenfalls beschreiben, indem man Modifikationen am Modell vornimmt, wie z.B. die Berücksichtigung von Zwischenschichten mit veränderten Löslichkeiten oder Diffusionskoeffizienten.

Im nächsten Abschnitt wird zunächst kurz der Meßaufbau vorgestellt. Danach folgt die Darstellung und Diskussion der Ergebnisse. 


\section{A.1 Elektrochemische Doppelzelle}

Neben der Möglichkeit der präzisen H-Beladung, die in Abschnitt 5.1.2 beschrieben ist, ist mit dem elektrochemischen Verfahren über die Bestimmung der elektromotorischen Kraft auch eine Konzentrationsbestimmung möglich. Dadurch eignet sich das Verfahren sehr gut für ein Permeationsexperiment zur Bestimmung der H-Diffusion.

Experimentell umgesetzt wird dies in einer elektrochemischen Doppelzelle, wie sie in Abb. A.1 dargestellt ist. Diese elektrochemische Methode zur Bestimmung der Wasserstofföslichkeit und zur Bestimmung des Diffusionskoeffizienten von Wasserstoff in Metallen wurde zuerst von Devanathan und Stachurski 1962 bei der Untersuchung des Palladium-Wasserstoff-Systems angewandt [71] und später von zahlreichen Autoren weiterentwickelt [72, 73].

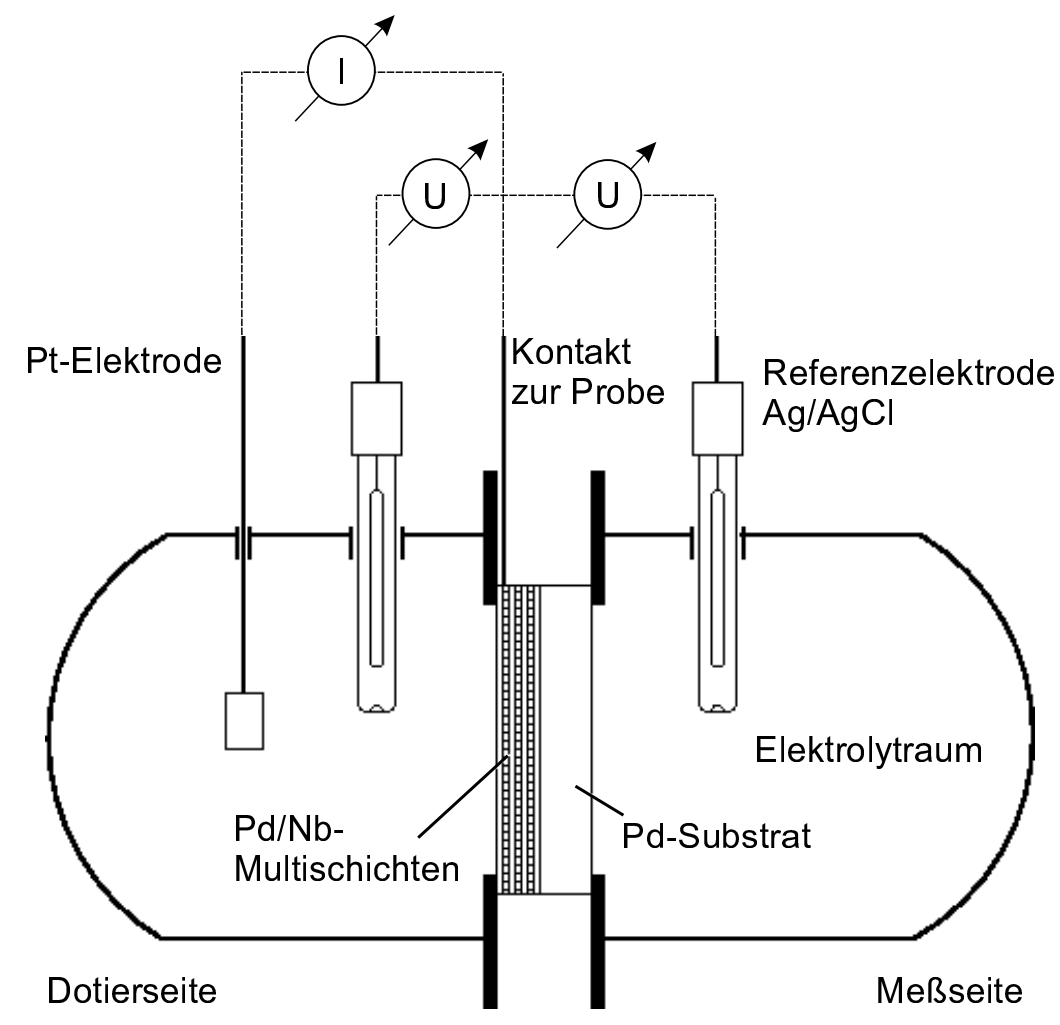

Abbildung A.1: Schematische Skizze der verwendeten elektrochemischen Doppelzelle.

Mit Hilfe der Doppelzelle gelingt es, einer der Probenoberflächen (Dotierseite) Wasserstoff anzubieten und gleichzeitig Änderungen der Wasserstoffkonzentration an der Rückseite der Probe (Meßseite) zu messen. Unter der Annahme von idealer H-Lösung und ausgehend von einer anfänglichen H-Konzentration $c_{0}$ in der Probe, läßt sich die Konzentrationsänderung $\Delta c$ an der Gegenseite über die 
Änderung der elektrochemischen Kraft $\Delta U$ bestimmen [29]:

$$
\Delta U=\frac{R T}{F} \cdot \ln \left(1+\frac{\Delta c}{c_{0}}\right) \approx \frac{R T}{F} \cdot \frac{\Delta c}{c_{0}}, \quad \text { mit } \quad c=\frac{n_{H}}{n_{M e}} .
$$

Die verwendete Apparatur ist detailliert in [65, 29, 64] beschrieben.

Auf der Rückseite der Probe wird sich die Wasserstoffkonzentration zeitlich verzögert ändern. Ist die Geometrie der Probe bekannt, so liefert die Bestimmung dieser zeitlichen Konzentrationsänderung die für die Diffusion des Wasserstoffes in der Probe charakteristischen Informationen.

\section{A.2 H-Permeation durch Multischichten}

Die Lösung der Diffusionsgleichungen in Einzel- und Multischichten für Konstantstrombedingungen ist in [61] hergeleitet und in Abschnitt 5.3.1 beschrieben. Als stationäre Lösung für die Permeation durch das gesamte Schichtpaket für lange Zeiten ergibt sich an der der Beladung abgewandten Seite des Schichtpaketes $L$ ein linearer Anstieg der Wasserstoffkonzentration mit der Beladungsdauer

$$
\Delta c(L, t)=\text { konst. } \cdot t
$$

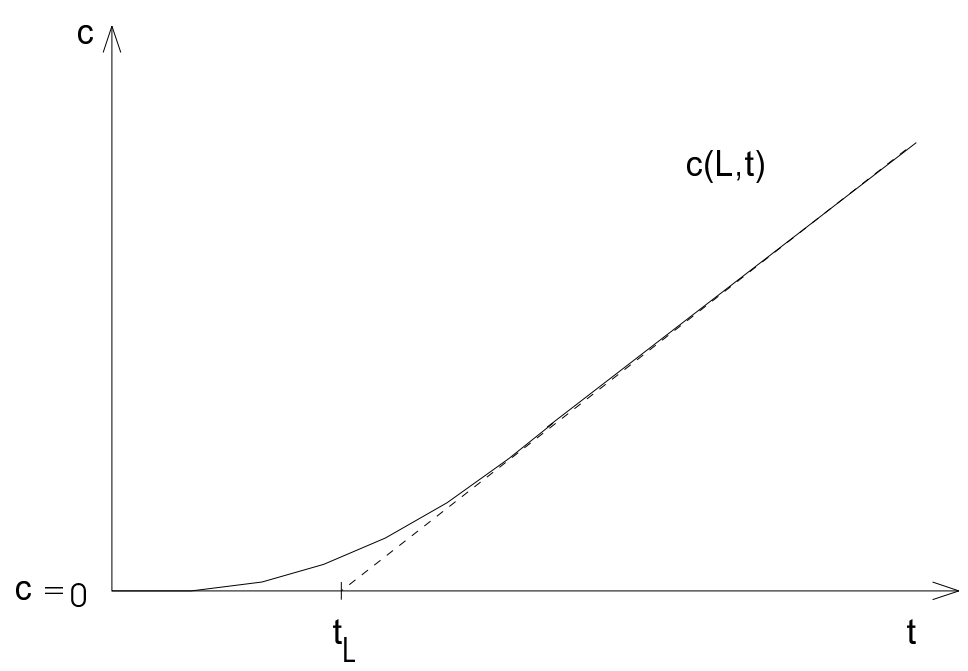

Abbildung A.2: Bestimmung der Durchbruchzeit $t_{L}$ aus dem linearen Anstieg der Konzentration an der Meßseite der Probe.

Extrapoliert man diesen linearen Anstieg der Konzentration für lange Zeiten auf die Ausgangskonzentration vor der Beladung, so erhält man mit dem Schnittpunkt zwischen der Grundlinie und der extrapolierten Geraden die charakteristische Durchbruchzeit, aus der der Diffusionskoeffizient ermittelt werden 
kann. Die exakte Lösung ist in [61] beschrieben. Eine Näherung für $\mathrm{Pd} / \mathrm{Nb}-$ Multischichsysteme auf Pd-Substraten ist in Abschnitt 5.3.1 skizziert

$$
t_{L}=3 \cdot t_{L}^{S u b}+t_{L}^{M L}
$$

wobei $t_{L}^{S u b}$ die Durchbruchzeit für das Pd-Substrat und $t_{L}^{M L}$ den Beitrag durch das Multischichsystem beschreibt.

Der Multischichterm $t_{L}^{M L}$ nimmt für hohe Löslichkeitsunterschiede und langsame Diffusion in dem Metall, das den Wasserstoff geringer löst, große Werte an

$$
t_{L}^{M L}=\frac{n^{2} \cdot a^{2}}{6 \cdot k \cdot D_{P d}} .
$$

Die H-Permeation durch Multischichten wird im Vergleich zur Permeation durch eine homogene Legierung der gleichen Gesamtdicke, die keine Lagenstruktur sondern einen mittleren Diffusionskoeffizienten und eine mittlere H-Löslichkeit besitzt, um mehrere Größenordnungen verzögert.

Die in Abschnitt 5.3.1 skizzierte Näherungslösung des Modells diente der Veranschaulichung des Verzögerungseffektes. Das Modell ist eine analytische Lösung des Permeationsproblems und erlaubt somit auch die Modellierung beliebiger Schichtsysteme ohne die gemachten Näherungen. Die exakte Lösung entnimmt man [61]. Sie wird im folgenden bei der Diskussion der Permeationsexperimente an $\mathrm{Pd} / \mathrm{Nb}-$ Schichtsystemen angewendet.

Elektrochemische Permeationsexperimente wurden an $\mathrm{Pd} / \mathrm{Nb}-\mathrm{Schichten}$ mit 4, 8 und 16 Doppelschichten durchgeführt. Als Substrat für die Multischichten wurde Pd-Folie der Firma Goodfellow mit einer Dicke von 12,5 $\mu \mathrm{m}$ und Flächen von ca. $1 \mathrm{~cm} \mathrm{x} 1 \mathrm{~cm}$ verwendet. Die mit Hilfe der gepulsten Laserseposition (s. Abschnitt 4.1.1) hergestellten Proben sind in Tabelle A.1 aufgeführt. Die Schichtdicken wurden wie in Kapitel 4 mit EDX und Profilometer bestimmt.

\begin{tabular}{|c|c|c|c|c|c|}
\hline \multicolumn{2}{|c|}{ Probe Nr. } & $\begin{array}{c}\text { Doppel- } \\
\text { lagen }\end{array}$ & $\begin{array}{c}\text { Doppelschicht- } \\
\text { dicke }[\mathrm{nm}]\end{array}$ & $\begin{array}{c}\text { Einzelschicht- } \\
\text { dicke Pd/Nb [nm] }\end{array}$ & $\begin{array}{c}\text { Gesamt- } \\
\text { dicke }[\mathrm{nm}]\end{array}$ \\
\hline \hline $\mathrm{Pd} / \mathrm{Nb}$ & 1 & 4 & 42,5 & $9 / 33,5$ & 170 \\
$\mathrm{Pd} / \mathrm{Nb}$ & 2 & 8 & 16 & $3 / 13$ & 128 \\
$\mathrm{Pd} / \mathrm{Nb}$ & 3 & 16 & 11,5 & $3 / 8,5$ & 184 \\
\hline
\end{tabular}

Tabelle A.1: Hergestellte Pd/Nb-Schichtsysteme auf Pd-Substraten.

Abbildung A.3 zeigt die gemessenen Durchbruchzeiten durch das Gesamtsystem einschließlich Pd-Substrat, die während der schrittweisen Beladung der Proben gewonnen wurden. Die H-Konzentration ist auf die Nb-Menge in den Proben bezogen, da bei kleinen H-Konzentrationen auf Grund des hohen Löslichkeitsunterschieds nahezu der gesamte Wasserstoff im Nb gelöst sein sollte. Man erkennt in allen Kurven bei geringen Konzentrationen sehr große Durchbruchzeiten, die auf eine sehr geringe H-Permeation schließen lassen. 
Diese großen Durchbruchzeiten bei geringen H-Konzentrationen können auf eine starke Bindung des Wasserstoffs an Defekte und Korngrenzen der Probe zurückgeführt werden, so daß dieser Wasserstoff nicht zur langreichweitigen Diffusion beitragen kann und die Permeation verzögert ist [29].

Sind diese tiefenergetischen Plätze abgesättigt, kommt es zu geringeren und von der H-Konzentration nahezu unabhängigen Durchbruchzeiten im Konzentrationsbereich von ca. 0,01-0,05 H/Nb. Dieser Bereich liegt in der $\alpha$-Phase des Nb-H-Systems (s. Abb. 2.3).

Für höhere Konzentrationen sinken die Durchbruchzeiten. In den Nb-Schichten bildet sich das Nb-Hydrid und die Permeation wird schneller. Dieser Konzentrationsbereich kann vom Modell nicht mehr beschrieben werden, weil die Löslichkeitsunterschiede an den Grenzflächen und die Diffusionskoeffizienten innerhalb der Schichten während der Phasenbildung nicht mehr bekannt sind.

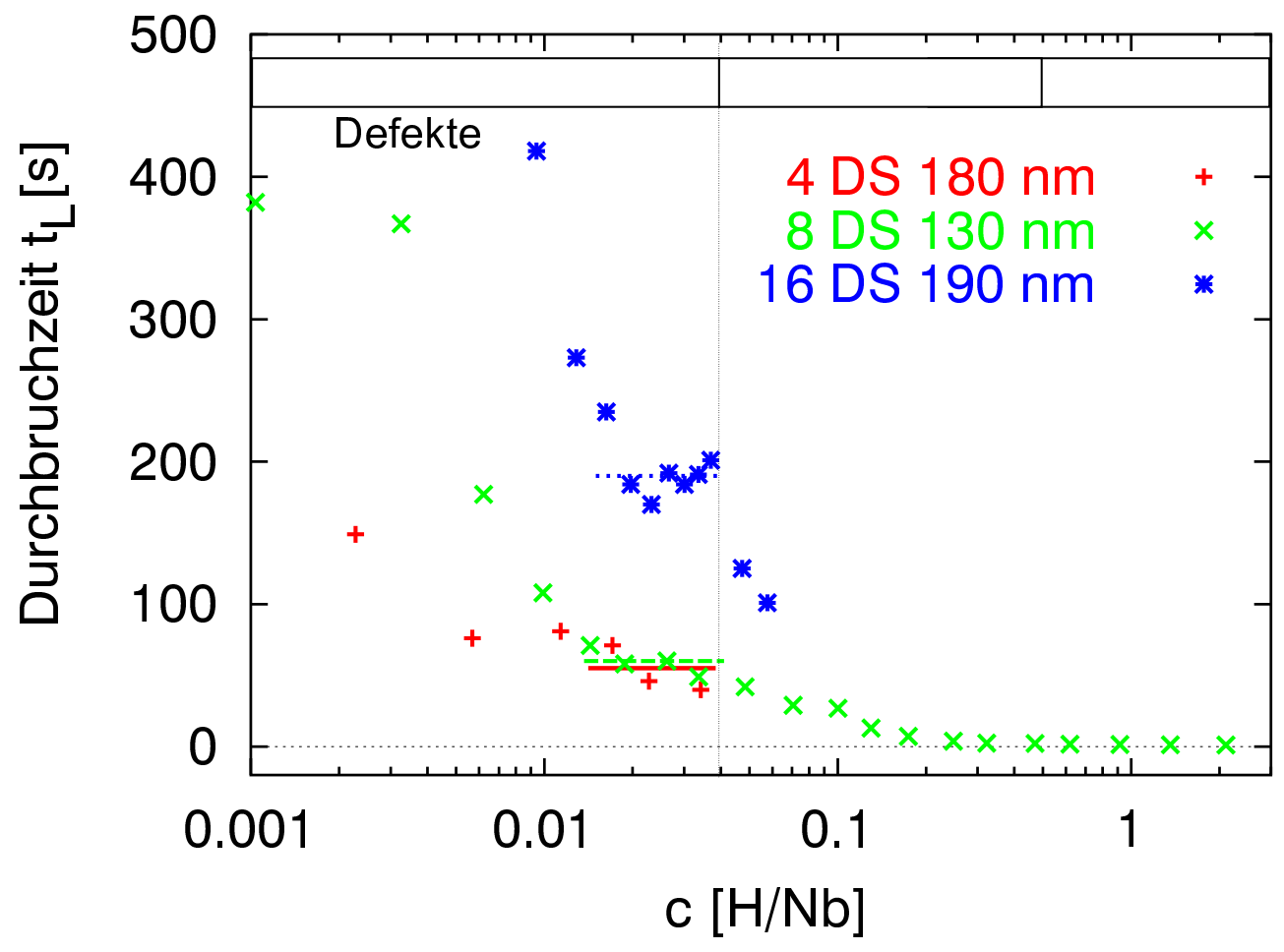

\begin{abstract}
Abbildung A.3: Mit der elektrochemischen Doppelzelle bestimmte Durchbruchzeiten $t_{L}$ der $H$-Permeation durch $\mathrm{Pd} / \mathrm{Nb}$-Multischichtsysteme auf Pd-Substrat aufgetragen gegen die H-Konzentration in den Nb-Schichten. Dargestellt sind die Meßwerte von Systemen mit 4, 8 und 16 Doppelschichten (DS) und die Gesamtdicke der Multischichtsysteme. Die Schichtgeometrien sind in Tab. A.1 angegeben.
\end{abstract}




\section{A.3 Diskussion der Ergebnisse}

Der Mittelwert der jeweiligen Durchbruchzeiten $t_{L}$ (experimentell), die im Bereich der $\alpha$-Phase des $\mathrm{Nb}-\mathrm{H}$-Systems gemessen wurden, sind in Abb. A.3 als waagerechte Linien eingezeichnet und in Tab. A.2 aufgeführt. Ebenfalls in Tab. A.2 angegeben sind die mit dem Modell berechneten Durchbruchzeiten $t_{L}$ (Modell), die man erwartet, wenn sich die H-Permeation durch die Diffusions- und Löslichkeitseigenschaften von Wasserstoff in massiven Metallen (s. Tabellen 2.2 und 2.4) beschreiben läßt. Die hierbei verwendeten Dicken sind in Tab. A.1 aufgeführt. Die Durchbruchzeiten liegen um mehr als eine Größenordnung unter den experimentellen Daten. Die H-Permeation zeigt somit im Experiment eine nochmals erheblich höhere Verzögerung als es das Modell mit den Literaturdaten vorhersagt.

\begin{tabular}{|c|c|c|c|c|}
\hline \multicolumn{2}{|c|}{ Probe Nr. } & $\begin{array}{c}\text { Doppel- } \\
\text { lagen }\end{array}$ & $\begin{array}{c}\left.t_{L} \text { (experimentell }\right) \\
\text { in } \alpha \text {-Nb-H-Phase }\end{array}$ & $\begin{array}{c}t_{L}(\text { Modell) mit Literaturdaten } \\
\text { für D und k (s. Tabn. 2.2 und 2.4) }\end{array}$ \\
\hline \hline $\mathrm{Pd} / \mathrm{Nb}$ & 1 & 4 & $55 \mathrm{~s}$ & $3,1 \mathrm{~s}$ \\
$\mathrm{Pd} / \mathrm{Nb}$ & 2 & 8 & $60 \mathrm{~s}$ & $2,8 \mathrm{~s}$ \\
$\mathrm{Pd} / \mathrm{Nb}$ & 3 & 16 & $190 \mathrm{~s}$ & $3,5 \mathrm{~s}$ \\
\hline
\end{tabular}

Tabelle A.2: Gegenüberstellung der Durchbruchzeiten $t_{L}$ aus dem Permeationsexperiment und aus dem Modell. Die experimentellen Werte sind im $\mathrm{H}$ Konzentrationsbereich bestimmt, der in Abb. A.3 mit den waagerechten Linien eingezeichnet ist ( $\alpha-\mathrm{Nb}$-H-Phase). Mit dem Permeationsmodell [61] wurde die Durchbruchzeit ausgerechnet. Man erkennt eine deutliche Verzögerung der $\mathrm{H}$ Permeation im Experiment im Vergleich zu den modellierten Daten.

Um die Ursache dieser zusätzlichen Verzögerung zu untersuchen, wurden Modifikationen am Modell vorgenommen, die den Einfluß von Schichtdicken- und Grenzflächeneffekten simulieren sollten. Dabei wurde jeweils ein Parameter des Modells verändert und die restlichen Schichtdicken, Diffusionskoeffizienten und H-Löslichkeiten konstant gehalten.

Zunächst wurde im Modell der H-Löslichkeitsunterschied zwischen $\mathrm{Nb}$ und Pd erhöht. Abbildung A.4 zeigt die Löslichkeitsunterschiede, die man im Modell einsetzen muß, um zu einer Übereinstimmung der Durchbruchzeiten mit den experimentellen Daten zu gelangen.

Man erkennt, daß mit zunehmender Anzahl der Doppelschichten die gemessene Verzögerung nur simuliert werden kann, wenn sich auch die Löslichkeit in den Nb-Schichten mit der Anzahl der Schichten erhöhen würde. Da die Einzelschichdicken in den untersuchten Schichtsystemen ebenfalls mit zunehmender Schichtanzahl von 33,5 nm im System mit 4 Doppelschichten auf 8,5 nm bei 16 Doppelschichten abnehmen, könnte dies ein Effekt der Nb-Schichtdicke sein. Das würde bedeuten, daß eine Abnahme der Schichtdicke die Löslichkeit in der Nb-Schicht erhöht und somit die H-Permeation verlangsamt. 


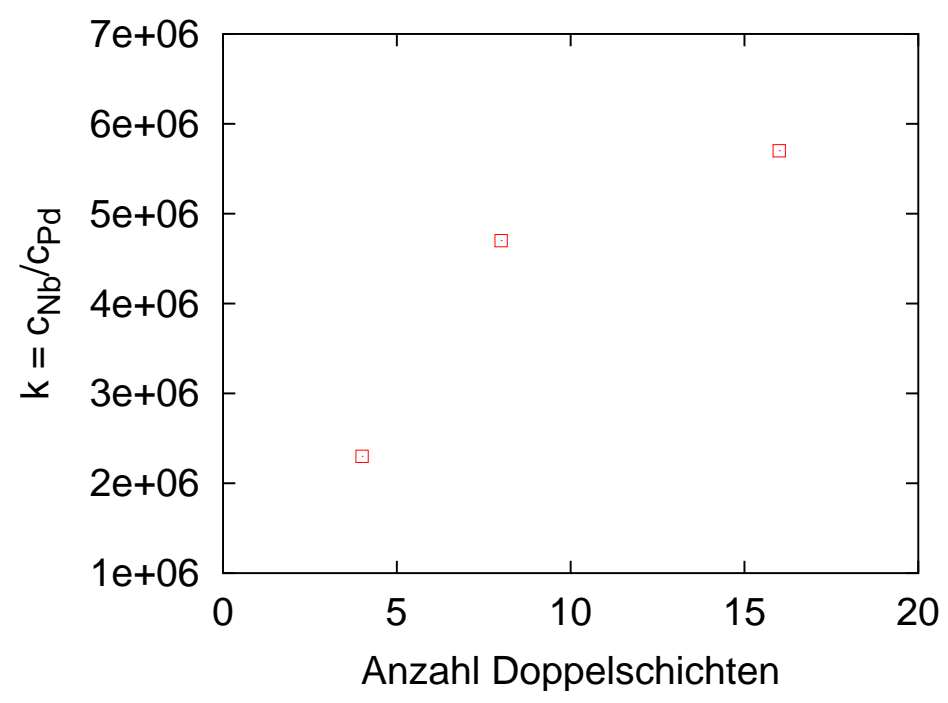

Abbildung A.4: Ins Permeationsmodell einzusetzender HLöslichkeitsunterschied zwischen $\mathrm{Nb}$ und $\mathrm{Pd}$, mit dem die experimentellen Durchbruchszeiten wiedergegeben werden (bei sonst unveränderten Parametern).

Andererseits deutet die zunehmende Verzögerung mit steigender Anzahl der Schichten auch auf einen Grenzflächeneffekt hin. Deswegen wurde im Modell an den Grenzflächen eine $1 \mathrm{~nm}$ breite Zwischenschicht angenommen (s. Skizze Abb. A.5). Dazu wurden die angrenzenden $\mathrm{Pd}$ - und Nb-Schichtdicken jeweils um 0,5 $\mathrm{nm}$ reduziert und die anderen Parameter innerhalb der Nb- und Pd-Schichten unverändert gelassen. Lediglich in der Zwischenschicht wurde die H-Löslichkeit und der Diffusionskoeffizient verändert.

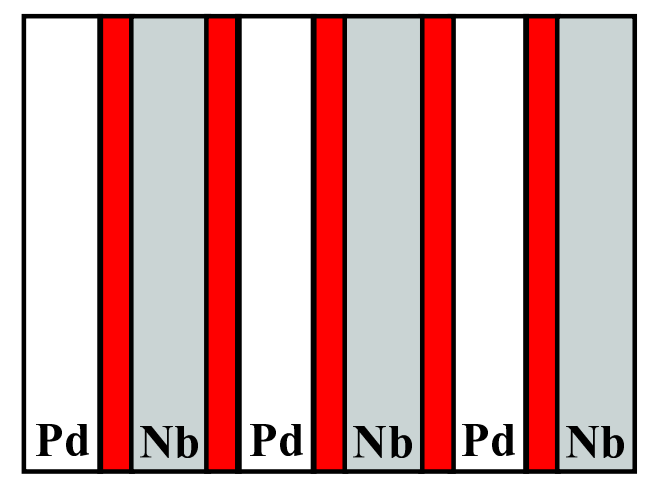

Abbildung A.5: Schematischer Aufbau der Multischichten für die Modellierung nach Einführung von Zwischenschichten (rot) zwischen den $\mathrm{Pd}$ - und $\mathrm{Nb}$ Schichten.

Abbildung A.6 zeigt die Löslichkeitsunterschiede, die man im Modell einsetzen muß, um zu einer Übereinstimmung der Durchbruchzeiten mit den experimentel- 
len Daten zu gelangen. Man erhält eine entsprechende Verzögerung der Permeation, wenn die H-Löslichkeit in der Zwischenschicht den Wert von Nb noch um ca. 3 Größenordnungen übersteigt. Der Diffusionskoeffizient in dieser hochlösenden Zwischenschicht spielt dem Modell zu Folge nur eine vernachlässigbare Rolle.

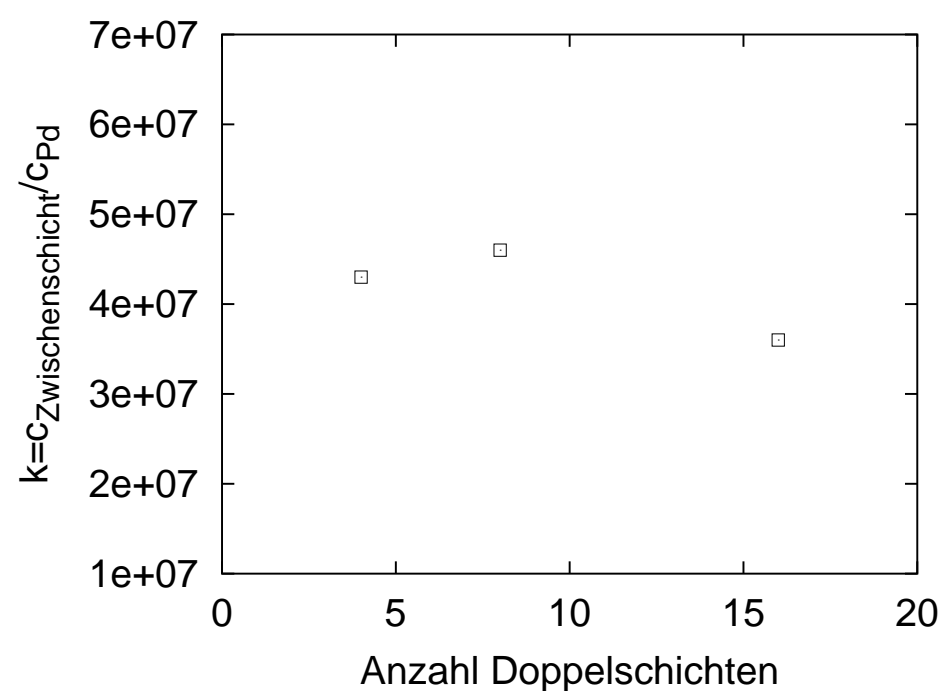

Abbildung A.6: Berechneter H-Löslichkeitsunterschied einer im Modell eingeführten Zwischenschicht zwischen den Pd-und Nb-Schichten (s. Abb. A.5) bezogen auf Pd. Die dargestellten Löslichkeitsunterschiede führen im Modell zu einer Übereinstimmung der Durchbruchzeiten mit den experimentellen Daten.

Durch die Einführung der Zwischenschicht im Modell verschwindet die Abhängigkeit der einzusetzenden H-Löslichkeit von der Anzahl der Doppelschichten. Das spricht dafür, daß durch die Berücksichtigung der Grenzflächen im Modell die Permeation zumindest qualitativ gut beschrieben wird.

Hervorzuheben ist, daß die eingeführte Zwischenschicht den Wasserstoff stärker lösen muß und nicht, wie bei den von Hjörvarsson formulierten elektronischen Effekten anzunehmen, geringer. Dies spricht im untersuchten $\mathrm{Pd} / \mathrm{Nb}$-System für einen Einfluß von Defekten an den Grenzflächen (s. Diskussion in Abschnitt 5.7.2).

Messungen an anderen Metallsystemen mit hohen Löslichkeitsunterschieden aber geringeren Gitterfehlpassungen wie z.B. Fe/V und Mo/V, bei denen die Defektdichte an der Grenzfläche stark reduziert sein sollte, könnten weitere Informationen liefern, ob eine gegenüber dem Modell beschleunigte Permeation durch elektronische Grenzflächeneffekte bzw. durch chemische Durchmischung beobachtet wird.

Im Rahmen der elektrochemischen Permeationsmessungen wurden auch Messungen zur Bestimmung der H-Permeation in den lateralen Richtungen der NbSchichten durchgeführt, um den Einfluß der Schichtdicke auf die H-Diffusion in der Schichtebene zu untersuchen. Auch hier können Abweichungen vom Verhalten 
in massiven Metallen erwartet werden. So sollten insbesondere Änderungen des Gitterparameters in dünnen Schichten, aber auch Grenzflächeneffekte in Multischichtsystemen deutlichen Einfluß auf die laterale Diffusion haben. Bisher konnten jedoch nur Messungen an relativ dicken Nb-Schichten im Bereich von 100 $\mathrm{nm}$ durchgeführt werden. Sie zeigen eine vernachlässigbare Abweichung des $\mathrm{H}-$ Diffusionskoeffizienten vom massiven $\mathrm{Nb}$. Experimente an dünneren Schichten konnten mit der zur Verfügung stehenden Apparatur nicht erfolgreich durchgeführt werden, da es durch zu hohe Stromdichten zu Phasenbildung im Nb-HSystem kam, die eine eindeutige Interpretation der Ergebnisse nicht zuließ. 


\section{Literaturverzeichnis}

[1] P. Grünberg. Acta Mat., 48, 239-251 (2000). 1

[2] W. Sproul. J. Vac. Sci. Techn. A, 12, 1595-1601 (1994). 1

[3] B. Ballot, F. Samuel und B. Farnoux. Phys. B, 198, 220-223 (1994). 1

[4] G. Song, M. Geitz, A. Abromeit und H. Zabel. Phys. Rev B, 54(19), 1409314101 (1996). 1, 5.7.2

[5] G. Song, A. Remhof, K. Theis-Bröhl und H. Zabel. Phys. Rev. Letters, 79(25), 5062 (1997). 1

[6] A. Abromeit, R. Siebrecht, G. Song, H. Zabel, F. Klose, D. Nagengast und A. Weidinger. J. Alloys and Compounds, 253-254, 58 (1997). 1

[7] U. Laudahn. Spannungen und Dehnungen von mit $H$ beladenen Nb-Einfachund Pd-Nb-Vielfachschichten. Doktorarbeit, Universität Göttingen (1998). $1,2.3 .1,4.2 .1,5.2,2,5.7 .2$

[8] P. Miceli, H. Zabel und J. Cunningham. Phys. Rev. Letters, 54(9), 917 (1985). 1

[9] P. Miceli und H. Zabel. Phys. Rev. Letters, 59(11), 1224 (1987). 1

[10] P. Miceli und H. Zabel. Z. Phys. B - Condensed Matter, 74, 457 (1989). 1

[11] C. Uher, J. Cohn, P. Miceli und H. Zabel. Phys. Rev. B, 36(1), 815 (1987). 1

[12] F. Stillesjö, B. Hjörvarsson und H. Zabel. Phys. Rev. B, 54(5), 3079-3083 (1996). 1

[13] G. Andersson, B. Hjörvarsson und H. Zabel. Phys. Rev. B, 55(23), 15905 (1997). 1

[14] D. Nagengast, J. Erxmeyer, F. Klose, C. Rehm, P. Kuschnerus, G. Dortmann und A. Weidinger. J. Alloys and Compounds, 231, 307-309 (1995). 1 
[15] Q. Yang, G. Schmitz, S. Fähler, H. Krebs und R. Kirchheim. Phys. Rev. B, 54, 9131 (1996). 1

[16] B. Hjörvarsson, J. Ryden, E. Karlsson, J. Birch und J.-E. Sundgren. Phys. Rev. B, 43(8), 6440 (1991). 1, 2.2, 5.7.2, 5.7.2, 6

[17] G. Andersson, B. Hjörvarsson und P. Isberg. Phys. Rev. B, 55(3), 1774 (1997). 1, 5.7.2, 5.7.2, 6

[18] B. Hjörvarsson, S. Olafsson, F. Stillesjö, E. Karlsson, J. Birch und J.-E. Sundgren. Z. Phys. Chem., 181, 343 (1993). 1

[19] B. Hjörvarsson, M. Vergnat, J. Birch, J.-E. Sundgren und B. Rodmacq. Phys. Rev. B, 50 (1994). 1

[20] S. Papadia, K. Karlsson, P. Nilsson und T. Jarlborg. Phys. Rev. B, 45, 1857-1868 (1992). 1, 5.7.2

[21] S. Olafsson, B. Hjörvarsson, F. Stillesjö, E. Karlsson, J. Birch und J.-E. Sundgren. Phys. Rev. B, 52(15), 10792 (1995). 1

[22] F. Stillesjö, S. Olafsson, P. Isberg und B. Hjörvarsson. J.Phys. Cond. Matter, 7, 8139-8150 (1995). 1

[23] B. Hjörvarsson, J. Ryden, T. Ericsson und E. Karlsson. Nucl. Instrum. Methods Phys. Res. Sect. B, 42, 257 (1989). 1

[24] W. Lanford. Nucl. Instrum. Methods Phys. Res. Sect. B, 66, 65 (1992). 1

[25] H. Züchner und T. Brüning. J. Alloys and Compounds, 231, 347-353 (1995). 1

[26] J. Scholz, H. Züchner, H. Paulus und K.-H. Müller. J. Alloys and Compounds, 253-254, 459-462 (1997). 1, 5.3.1, 5.6, 6

[27] H. Züchner, J. Kintrup, R. Dobrileit und I. Untiedt. J. Allloys and Compounds, 293-295, 202-212 (1999). 1

[28] R. Becker. Theorie der Wärme. Springer-Verlag, Berlin Heidelberg (1985). 2.1

[29] R. Kirchheim. Prog. Mat. Sci., 32, 261-325 (1988). 2.1, 2.1, 2.2, A.1, A.1, A.2

[30] A. Sieverts. Z. Metallkd., 2, 37 (1929). 2.1

[31] E. Fromm und E.Gebhardt. Gase und Kohlenstoff in Metallen, Band 26 von Reine und angewandte Metallkunde in Einzeldarstellungen. Springer-Verlag, Berlin Heidelberg (1976). 2.1, 2.1, 2.1, 3, 5.5.1, 5.7.2 
[32] R. Griessen und T. Riesterer. Hydrogen in Intermetallic Compounds I, Band 63 von Topics in Applied Physics, Kapitel 6. Springer-Verlag, Berlin Heidelberg New York (1988). Hrsg. L. Schlapbach. 2.1, 2.1, 5.7.2

[33] Y. Fukai. The Metal-Hydrogen System. Springer-Verlag, Berlin Heidelberg (1993). 2.1, 2.1, 2.1, 5.5.1

[34] P. Haasen. Physikalische Metallkunde. Springer-Verlag, Berlin Heidelberg, 3. Ausgabe (1994). 2.1

[35] T. Schober und H. Wenzl. Hydrogen in Metals II, Band 29 von Topics in Applied Physics, Kapitel 2. Springer-Verlag, Berlin Heidelberg (1978). Hrsg. G. Alefeld und J. Völkl. 2.1, 2.3.2, 2.2, 2.3, 5.7.2

[36] M. Maxelon. Doktorarbeit, Universität Göttingen (2000). 2.1

[37] T. Mütschele. Löslichkeit und Diffusion von Wasserstoff in nanokristallinen Palladium. Doktorarbeit, Max-Plank-Institut Stuttgart (1987). 2.1

[38] W. Nix und R. Mehl. Metallurgical Transactions A, 20A, 2217 (1989). 2.3.1

[39] P. Isberg, B. Hjörvarsson, R. Wäppling, E. Svedberg und L. Hultman. Vacuum, 48(5), 483 (1997). 2.3.1, 5.7.2, 5.7.2

[40] T. B. Massalski. Binary Alloy Phase Diagrams, Band 3. ASM international, 2. Ausgabe (1990). 2.3.2, 2.3.3, 5.7.2

[41] D. Isheim. Feldionen- und Elektronenmikroskopie der Entmischung einer Fe20 At.\% Mo-Legierung. Doktorarbeit, Universität Göttingen (1995). 2.3.3

[42] E. W. Müller und T. Tsong. Field Ion Microscopy. American Elsevier Publishing Company, New York (1969). 3.1.1

[43] G. Geber, T. Al-Kassab, D. Isheim, R. Busch und P. Haasen. Z. Metallkd., 83(6), 449-456 (1992). 3.1.1, 3.1.2

[44] M. Miller und G. Smith. Atom Probe Microanalysis. Materials Research Society, Pittsburgh PA (1989). 3.1.2, 3.1.2

[45] M. Miller, A. Cerezo, M. Hetherington und G. Smith. Atom Probe Microanalysis. Clarendon Press, Oxford (1996). 3.1.2

[46] A. Bostel, D. Blavette, A. Menand und J. Sarreau. J. de Phys. C8, 50, 501 (1989). 3.1 .2

[47] D. Blavette, B. Deconihout, A. Bostel, J. Sarreau, M. Bouet und A. Menand. Rev. Sci. Instrum., 64(11), 2911 (1993). 3.1 .2 
[48] T. Al-Kassab, H. Wollenberger, G. Schmitz und R. Kirchheim. High Imaging and Spectroscopy of Materials, Kapitel "Tomography by Atom Probe". Springer Verlag, Berlin, Heidelberg, New York (to be published). Hrsg. M. Rühle und F. Ernst. 3.1.2, 3.1.2, 3.1.2, 5.7.1

[49] H. Gnaser und H. Oechsner. Fresenius J. Anal. Chem., 341, 54-56 (1991). $3.2,4.3 .1,5.3$

[50] H. Gnaser. J. Vac. Sci. Technol. A, 12(2), 452-456 (1994). 3.2, 4.3.1, 5.3

[51] J. Bankmann. Untersuchungen zum Einsatz der Sekundärionenmassenspektrometrie bei der Tiefenprofilierung dünner Einzel- und Vielfachschichten. Diplomarbeit, Universität Göttingen (1998). 3.2, 5.3

[52] S. Fähler. Doktorarbeit, Universität Göttingen (1998). 4.1.1, 1

[53] M. Störmer. Doktorarbeit, Universität Göttingen (1998). 4.1.1

[54] M. Störmer, K. Sturm, M. Weisheit, J. Winkler, S. Kahl, P. Kesten, A. Pundt, S. Senz, und H. Krebs. Appl. Phys. A, 69(Suppl.), S455-S457 (1999). 4.1 .1

[55] A. Pundt. Untersuchungen zur Entmischung in Co-20 At. \% Cr-Legierungen mit Hilfe der analytischen Feldionenmikroskopie. Doktorarbeit, Universität Göttingen (1995). 4.1.2, 4.2.2

[56] M. Dornheim Private Mitteilungen. 4.2.1, 4.1

[57] C. Borchers, U. Laudahn, A. Pundt, S. Fähler, H. Krebs und R. Kirchheim. Phil. Mag. A, 80(3), 543-553 (2000). 4.2.1, 4.2

[58] R. Busch. Analytische Feldionenmikroskopie der Reaktion in Zr-Co Doppelschichten. Doktorarbeit, Universität Göttingen (1992). 4.2 .2

[59] F. Wang. Struktur und Morphologie innenoxidierter Pd-Mg-Legierungen. Doktorarbeit, Universität Göttingen (1999). 5.1.1

[60] F. Hartung. Doktorarbeit, Universität Göttingen (2000). 5.3

[61] G. Schmitz, P. Kesten, R. Kirchheim und Q. Yang. Phys. Rev. B, 58(11), 7333-7339 (1998). 5.3, 5.3.1, 5.3.1, 5.3.1, 5.3.1, 5.7.2, 6, A, A.2, A.2, A.2, A.2

[62] L. Schlapbach. Hydrogen in Intermetallic Compounds II, Band 67 von Topics in Applied Physics, Kapitel 1. Springer-Verlag, Berlin Heidelberg New York (1992). Hrsg. L. Schlapbach. 5.3.1 
[63] R. Ash, R. Barrer und J. Petropoulos. Brit. J. Appl. Physics, 14, 854 (1963). 5.3 .1

[64] P. Kesten. Zur Diffusion von Wassertoff in metallischen Multischichten. Diplomarbeit, Universität Göttingen (1996). 5.3.1, A, A.1

[65] U. Stolz. Löslichkeit, Diffusion und partielles Molvolumen von Wasserstoff in amorphen Pd-Si-Legierungen. Doktorarbeit, Universität Stuttgart (1984). 5.3.1, A.1

[66] H. Wipf. Hydrogen in Metals III, Band 73 von Topics in Applied Physics, Kapitel 3. Springer-Verlag, Berlin Heidelberg New York (1997). Hrsg. H. Wipf. 5.5.1, 5.21

[67] K. Christmann. Molecular Phys., 66, 1 (1989). 5.6

[68] R. Behm, V. Penka, M. Cattania, K. Christmann und E. Ertl. J. Chem. Phys., 78(12), 7486 (1983). 5.6

[69] C. Kluthe. Diplomarbeit, Universität Göttingen (2000). 5.7.1

[70] P. Kesten, M. Weisheit, A. Pundt, H. Krebs und R. Kirchheim. to be published, (2000). 5.7 .2

[71] M. Devanathan und Z. Stachurski. Proc. Roy. Soc. London, 270, 90 (1962). A.1

[72] H. Züchner. Z. Naturforschung, 25a, 1490-1496 (1970). A.1

[73] R. Kirchheim und R. McLellan. J.Electrochemical Society, 127, 2419 (1980). A.1 


\section{Danksagung}

An erster Stelle möchte ich Herrn Prof. Dr. Kirchheim für die Möglichkeit danken, meine Doktorarbeit am Institut für Materialphysik anfertigen zu dürfen. Sein großes Interesse an meiner Arbeit und seine ständige Gesprächsbereitschaft haben mir sehr geholfen.

Ganz besonders danken möchte ich Frau Dr. A. Pundt für die hervorragende Betreuung meiner Arbeit und ihre unerschütterliche Zuversicht, daß die Spitzen halten.

Auch Herrn Dr. Guido Schmitz danke ich für seine ständige Ansprechbereitschaft und Unterstützung. Sein Einsatz für den Aufbau und den reibungslosen Betrieb der tomographischen Atomsonde hat diese Arbeit erst möglich gemacht.

Den anderen Mitgliedern der Wasserstoff-Gruppe M. Dornheim, M. Maxelon, K. Nörthemann, M. Shtaya, J. Mertins und der FIM-Gruppe Ch. Lang, J. Schleiwies, T. Jeske, B. Färber, T. Al-Kassab, Ch. Kluthe und P. Choi danke ich für die sehr gute Arbeitsatmosphäre und die vielen Anregungen und Hilfestellungen.

M. Weisheit und Dr. H.U. Krebs danke ich für die Präparation der laserdeponierten Schichtsysteme und die gute Zusammenarbeit. Ch. Lang, K. Nörthemann und M. Hahn danke ich für Ihre Unterstützung bei der Präparation der gesputterten Schichtsysteme.

J. Bankmann und Dr. P Wilbrandt danke ich für ihre Unterstützung bei den SIMS-Messungen und ihren Einsatz beim Aufbau und Betrieb des SIMS.

Ich danke allen Mitgliedern der Feinmechanik- und der Elektrowerkstatt und den Laboranten insbesondere M. Rothämel, F. Köhler, D. Bollensen, T. Nägel, K. Ahlborn, T. Schulz für die sofortige und unkomplizierte Hilfe bei Problemen mit den Meßapparaturen.

Ph. Quan danke ich für ihre sehr guten und schnellen Fotoarbeiten.

Ich danke den vielen anderen Institutsangehörigen, die durch ihre nette Art, zum angenehmen Arbeitsklima im Institut beigetragen haben.

Ein spezieller Dank geht an Joachim Bankmann, Klaus Krug, Ruth Lüke, Iris Böhm, Christian Lang, Frank Hartung und an die "Ehemaligen" Jörg Ewert, Holger Voß und Uwe Laudahn für ihre ständige Hilfsbereitschaft und ihr Interesse nicht nur am Fortgang meiner Arbeit.

Meinen Eltern danke ich dafür, daß sie mir dieses Studium ermöglicht haben und für ihr Vertrauen in dieses teure Unternehmen.

Mein größter Dank jedoch geht an meine Frau Stefanie, ohne deren Geduld und Unterstützung diese Arbeit nicht möglich gewesen wäre, und an meine beiden Söhne, die das "Papa muß nochmal ins Institut" meist ohne zu murren hingenommen haben. 